\title{
ON EVEN RAINBOW OR NONTRIANGULAR DIRECTED CYCLES
}

\author{
ANDRZEJ CZYGRINOW, THEODORE MOLLA, BRENDAN NAGLE, AND ROY OURSLER
}

\begin{abstract}
Let $G=(V, E)$ be an $n$-vertex edge-colored graph. In 2013, H. Li proved that if every vertex $v \in V$ is incident to at least $(n+1) / 2$ distinctly colored edges, then $G$ admits a rainbow triangle. We establish a corresponding result for fixed even rainbow $\ell$-cycles $C_{\ell}$ : if every vertex $v \in V$ is incident to at least $(n+5) / 3$ distinctly colored edges, where $n \geq n_{0}(\ell)$ is sufficiently large, then $G$ admits an even rainbow $\ell$-cycle $C_{\ell}$. This result is best possible whenever $\ell \not \equiv 0(\bmod 3)$. Correspondingly, we also show that for a fixed (even or odd) integer $\ell \geq 4$, every large $n$-vertex oriented graph $\vec{G}=(V, \vec{E})$ with minimum outdegree at least $(n+1) / 3$ admits a (consistently) directed $\ell$-cycle $\vec{C}_{\ell}$. Our latter result relates to one of Kelly, Kühn, and Osthus, who proved a similar statement for oriented graphs with large semi-degree. Our proofs are based on the stability method.
\end{abstract}

\section{INTRODUCTION}

An edge-colored graph is a pair $(G, c)$, where $G=(V, E)$ is a graph and $c: E \rightarrow P$ is a function mapping edges to some palette of colors $P$. A subgraph $H \subseteq G$ is a rainbow subgraph if the edges of $H$ are distinctly colored by $c$. We consider degree conditions ensuring the existence of rainbow cycles $C_{\ell}$ in $(G, c)$ of fixed even length $\ell \geq 4$. To that end, a vertex $v \in V$ in an edge-colored graph $(G, c)$ has $c$-degree $\operatorname{deg}_{G}^{c}(v)$ given by the number of distinct colors assigned by $c$ to the edges $\{v, w\} \in E$, where we set $\delta^{c}(G)=\min _{v \in V} \operatorname{deg}_{G}^{c}(v)$. The following result of $\mathrm{H}$. Li [16] motivates the main results of our paper.

Theorem $1.1\left(\mathrm{H}\right.$. Li, 2013). Let $(G, c)$ be an n-vertex edge-colored graph. If $\delta^{c}(G) \geq(n+1) / 2$, then $(G, c)$ admits a rainbow 3-cycle $C_{3}$.

A rainbow $K_{\lfloor n / 2\rfloor,\lceil n / 2\rceil}$ shows that Theorem 1.1 is best possible.

Our first result ensures rainbow cycles of fixed even length.

Theorem 1.2. There exists an absolute constant $\alpha>0$ so that, for every even integer $\ell \geq 4$, every edge-colored graph $(G, c)$ on $n \geq n_{0}(\ell)$ many vertices satisfying

$$
\delta^{c}(G) \geq\left\{\begin{array}{cl}
\left(\frac{1}{3}-\alpha\right) & \text { if } \ell \equiv 0(\bmod 3) \\
\frac{n+5}{3} & \text { if } \ell \neq \equiv 0(\bmod 3)
\end{array}\right.
$$

admits a rainbow $\ell$-cycle $C_{\ell}$.

Theorem 1.2 is best possible for $\ell \not \equiv 0(\bmod 3)$, which we verify at the end of the Introduction. We prove Theorem 1.2 in Section 2 using the stability method.

Remark 1.3. In a related paper [5], we establish an analogue of Theorem 1.2 for fixed odd integers $\ell \geq 3$. In particular, we show that for large integers $n \geq n_{0}(\ell)$, H. Li's condition $\delta^{c}(G) \geq(n+1) / 2$ ensures rainbow $\ell$-cycles $C_{\ell}$ in $(G, c)$, which is again best possible by a rainbow $K_{\lfloor n / 2\rfloor,\lceil n / 2\rceil}$.

We also consider an analogue of Theorem 1.2 for oriented graphs $\vec{G}=(V, \vec{E})$, i.e., those for which $\vec{E} \subset V \times V$ satisfies the rule that $(u, v) \in \vec{E}$ forbids $(v, u) \in \vec{E}$. Here, we seek a directed or consistently

Date: December 5, 2019.

2000 Mathematics Subject Classification. 05C20, 05C35, 05C38.

Key words and phrases. edge-colored, rainbow subgraph, directed graphs.

The first author was partially supported by Simons Foundation Grant \#521777.

The second author was partially supported by NSF Grants DMS 1500121 and DMS 1800761 .

The third author was partially supported by NSF Grant DMS 1700280. 
oriented $\ell$-cycle $\vec{C}_{\ell}$, whose vertices $V\left(\vec{C}_{\ell}\right)$ may be ordered $\left(v_{0}, \ldots, v_{\ell-1}\right)$ so that $\left(v_{i}, v_{i+1}\right) \in \vec{E}$ for all $i \in \mathbb{Z}_{\ell}$. In this context, we may take $\ell \geq 4$ to be even or odd.

Theorem 1.4. For every fixed integer $\ell \geq 4$, whether even or odd, every oriented graph $\vec{G}=(V, \vec{E})$ on $n \geq n_{0}(\ell)$ many vertices with minimum out-degree $\delta^{+}(\vec{G}) \geq(n+1) / 3$ admits a directed $\ell$-cycle $\vec{C}_{\ell}$.

We prove Theorem 1.4 in Section 2 using ideas similar to that of Theorem 1.2. Note that Theorem 1.4 is best possible for every $\ell \not \equiv 0(\bmod 3)$, as seen by the blow-up $\vec{G}=(V, \vec{E})$ of a directed triangle:

let $V=V_{0} \cup V_{1} \cup V_{2}$ be a partition, and let $\vec{E}=\left(V_{0} \times V_{1}\right) \cup\left(V_{1} \times V_{2}\right) \cup\left(V_{2} \times V_{0}\right)$,

where $\left|V_{2}\right| \leq\left|V_{1}\right| \leq\left|V_{0}\right| \leq\left|V_{2}\right|+1$. Here, $\delta^{+}(\vec{G})=\left|V_{2}\right| \geq((n+1) / 3)-1$.

Note that Theorem 1.4 omits the case $\ell=3$, which is the triangular case of the Caccetta-Häggkvist conjecture (cf. [3, 8]) and is beyond the reach of our methods. We also mention that Theorem 1.4 relates to the following result of Kelly, Kühn, and Osthus [12].

Theorem 1.5 (Kelly, Kühn, Osthus, 2010). For every integer $\ell \geq 4$ and for every integer $n \geq 10^{10} \ell$, every $n$-vertex oriented graph $\vec{G}=(V, E)$ with $\delta_{0}(\vec{G})=\min \left\{\delta^{+}(\vec{G}), \delta^{-}(\vec{G})\right\} \geq(n+1) / 3$ contains a directed $\ell$-cycle $\vec{C}_{\ell}$. Moreover, every vertex $v \in V$ belongs to a directed $\ell$-cycle $\overrightarrow{\vec{C}}_{\ell}$.

The remainder of this paper is organized as follows. In Section 2, we prove both Theorems 1.2 and 1.4 . For these proofs, we need upcoming Lemmas 2.6 and 2.9, which (in a sense made precise later) distinguish whether or not a given context is extremal. We prove Lemma 2.6 in Sections 35 where we also prove supplemental results needed along the way. We prove Lemma 2.9 in Sections 6 8, where again we prove supplemental results needed along the way. We conclude this Introduction by verifying the sharpness of Theorem 1.2 when $\ell \not \equiv 0(\bmod 3)$.

1.1. Theorem 1.2 is sharp for $\ell \not \equiv 0(\bmod 3)$. Fix an integer $\ell \not \equiv 0(\bmod 3)$, which in the constructions below can be even or odd. Let $V=V_{0} \cup V_{1} \cup V_{2}$ be a partition of an $n$-element set $V$, where for optimality we take $\lfloor n / 3\rfloor=m=\left|V_{2}\right| \leq\left|V_{1}\right| \leq\left|V_{0}\right| \leq\left|V_{2}\right|+1$. Let $G=(V, E)$ be given by the complete 3 -partite graph $K\left[V_{0}, V_{1}, V_{2}\right]$. We now distinguish the cases $n, \ell(\bmod 3)$.

Case $1(n \not \equiv 2(\bmod 3))$. Define $c_{+}: E \rightarrow V$ by setting, for each $i \in \mathbb{Z}_{3}$ and $\left(v_{i}, v_{i+1}\right) \in V_{i} \times V_{i+1}$,

$$
c_{+}\left(\left\{v_{i}, v_{i+1}\right\}\right)=v_{i+1} .
$$

We say this same edge $e=\left\{v_{i}, v_{i+1}\right\} \in E$ is of type $i$, and we write $t(e)=i$ for its type. We write a fixed $\ell$-cycle $C_{\ell}$ in $G$ by a cyclic ordering $\left(e_{0}, e_{1}, \ldots, e_{\ell-1}\right)$ of its consecutive edges. A consecutive such pair $\left(e_{k}, e_{k+1}\right)$ is a reversal when $e_{k}$ and $e_{k+1}$ are of the same type $t\left(e_{k}\right)=t\left(e_{k+1}\right)=i \in \mathbb{Z}_{3}$, where $\left(e_{k}, e_{k+1}\right)$ is a backward reversal when $e_{k} \cap e_{k+1} \in V_{i+1}$, and $\left(e_{k}, e_{k+1}\right)$ is a forward reversal when $e_{k} \cap e_{k+1} \in V_{i}$. Since $C_{\ell}$ is a cycle, the number of backward reversals is the number of forward reversals, and $C_{\ell}$ admits backward reversals lest $\ell \equiv 0(\bmod 3)$. Fix an arbitrary backward reversal $\left(e_{k}, e_{k+1}\right)$ of $C_{\ell}$, where $k \in \mathbb{Z}_{\ell}$, where $t\left(e_{k}\right)=t\left(e_{k+1}\right)=i \in \mathbb{Z}_{3}$, and where $e_{k} \cap e_{k+1}=\left\{v_{i+1}\right\} \subset V_{i+1}$. Then

$$
c_{+}\left(e_{k}\right) \stackrel{2}{=} v_{i+1} \stackrel{2}{=} c_{+}\left(e_{k+1}\right)
$$

whence $C_{\ell}$ isn't rainbow. We observe from (2) that $\operatorname{deg}_{G}^{c_{+}}\left(v_{i}\right)=1+\left|V_{i+1}\right|$ holds for each fixed $i \in \mathbb{Z}_{3}$ and for each fixed $v_{i} \in V_{i}$. Indeed, an incident edge $e=\left\{v_{i}, v_{j}\right\} \in E$ is assigned the fixed color $c_{+}(e)=v_{i}$ when $v_{j} \in V_{i-1}$, and is assigned the variable color $c_{+}(e)=v_{j}$ among all $\left|V_{i+1}\right|$ many possible $v_{j} \in V_{i+1}$. As such, $\delta^{c_{+}}(G)=\operatorname{deg}_{G}^{c_{+}}\left(v_{1}\right)=m+1$ is achieved by any vertex $v_{1} \in V_{1}$, while $\lceil(n+5) / 3\rceil=m+2$ is ensured by $n \not \equiv 2(\bmod 3)$.

Case $2(n \equiv 2, \ell \equiv 1(\bmod 3))$. Here, $n \equiv 2(\bmod 3)$ ensures that $\left|V_{0}\right|=\left|V_{1}\right|=m+1$. Fix a perfect matching $M=\left\{\left\{x_{1}, y_{1}\right\}, \ldots,\left\{x_{m+1}, y_{m+1}\right\}\right\}$ of $G\left[V_{0}, V_{1}\right]=K\left[V_{0}, V_{1}\right]$, where $V_{0}=\left\{x_{1}, \ldots, x_{m+1}\right\}$ and 
$V_{1}=\left\{y_{1}, \ldots, y_{m+1}\right\}$, and fix a symbol $\star \notin V$. Define $c_{M}: E \rightarrow\{\star\} \cup V$ by

$$
c_{M}(e)=\left\{\begin{array}{cc}
c_{+}(e) & \text { if } e \in E \backslash E_{G}\left[V_{0}, V_{1}\right], \\
\star & \text { if } e \in M, \\
x_{b} & \text { if } e=\left\{x_{a}, y_{b}\right\} \in E_{G}\left[V_{0}, V_{1}\right] \backslash M .
\end{array}\right.
$$

We observe from (4) that $\left(G, c_{M}\right)$ is $(m+2)$-color-regular, while $\lceil(n+5) / 3\rceil=m+3$ is ensured by $n \equiv 2(\bmod 3)$. Indeed, as before in Case 1, a vertex $v_{2} \in V_{2}$ has color-degree $\operatorname{deg}_{G}^{c_{M}}\left(v_{2}\right)=\operatorname{deg}_{G}^{c_{+}}\left(v_{2}\right)=$ $1+\left|V_{0}\right|=m+2$. Less easily, fix $x_{a} \in V_{0}$ and fix an incident edge $x_{a} \in e \in E$. If $e \cap V_{2} \neq \emptyset$, then $e$ is assigned the fixed color $c_{M}(e)=c_{+}(e)=x_{a}$, and if $e=\left\{x_{a}, y_{a}\right\} \in M$, then $e$ is assigned the fixed color $c_{M}(e)=\star$. Otherwise, $e=\left\{x_{a}, y_{b}\right\} \in E\left[V_{0}, V_{1}\right] \backslash M$ for some $y_{a} \neq y_{b} \in V_{1}$, whence $e$ is assigned the variable color $c_{M}(e)=x_{b}$ among all $\left|V_{1}\right|-1=m$ many possible $y_{b} \in V_{1} \backslash\left\{y_{a}\right\}$. Similarly, fix $y_{b} \in V_{1}$, and fix an incident edge $y_{b} \in e \in E$. If $e=\left\{x_{b}, y_{b}\right\} \in M$, then $e$ is assigned the fixed color $c_{M}(e)=\star$, and if $e=\left\{x_{a}, y_{b}\right\} \in E\left[V_{0}, V_{1}\right] \backslash M$ for some $x_{b} \neq x_{a} \in V_{0}$, then $e$ is assigned the fixed color $c_{M}(e)=x_{b}$. Otherwise, $e=\left\{y_{b}, v_{2}\right\} \in E\left[V_{1}, V_{2}\right]$ for some $v_{2} \in V_{2}$, whence $e$ is assigned the variable color $c_{M}(e)=c_{+}(e)=v_{2}$ among all $\left|V_{2}\right|=m$ many possible $v_{2} \in V_{2}$.

We now observe that $\left(G, c_{M}\right)$ avoids rainbow $\ell$-cycles $C_{\ell}$. For that, fix an $\ell$-cycle $C_{\ell}=\left(e_{0}, \ldots, e_{\ell-1}\right)$ of $G$ with backward reversal $\left(e_{k}, e_{k+1}\right)$, where $k \in \mathbb{Z}_{\ell}$. For $C_{\ell}$ to be rainbow, we claim that $G$ must assume the color $\star$ within the backward reversal $\left(e_{k}, e_{k+1}\right)$. Indeed, let $t\left(e_{k}\right)=t\left(e_{k+1}\right)=i \in \mathbb{Z}_{3}$, and let $e_{k} \cap e_{k+1}=\left\{v_{i+1}\right\} \subset V_{i+1}$. For $C_{\ell}$ to be rainbow, $i=0$ is necessary lest (3) holds, so write $v_{i+1}=y_{1} \in V_{1}$. Since $M$ is a matching, at most one of $e_{k}, e_{k+1} \in M$, but for $C_{\ell}$ to be rainbow, at least one such containment is necessary (as claimed) lest (4) gives $c_{M}\left(e_{k}\right)=x_{1}=c_{M}\left(e_{k+1}\right)$. Now, for $C_{\ell}$ to be rainbow, the following are necessary:

(a) $e_{k} \in M$ implies $\left(e_{k-1}, e_{k}\right)$ is a forward reversal, lest

$$
c_{M}\left(e_{k-1}\right) \stackrel{4}{=} c_{+}\left(e_{k-1}\right) \stackrel{(2)}{=} x_{1} \stackrel{4}{=} c_{M}\left(e_{k+1}\right) \text {; }
$$

(b) $e_{k+1} \in M$ implies $\left(e_{k+1}, e_{k+2}\right)$ is a forward reversal, lest

$$
c_{M}\left(e_{k+2}\right) \stackrel{(4)}{=} c_{+}\left(e_{k+2}\right) \stackrel{(2)}{=} x_{1} \stackrel{4}{=} c_{M}\left(e_{k}\right)
$$

Either way, $C_{\ell}$ has further backward reversals (assuming $\star$ again) lest $\ell \equiv 2(\bmod 3)$.

Case $3(n, \ell \equiv 2(\bmod 3))$. We first slightly alter the graph $G=K\left[V_{0}, V_{1}, V_{2}\right]$ above, as follows. Fix $x \in V_{0}$ and $y \in V_{1}$ so that $U_{0}=V_{0} \backslash\{x\}, U_{1}=V_{1} \backslash\{y\}$, and $U_{2}=V_{2}$ all have size $m$. Define $\hat{E}$ by the rule that, for each $\{u, v\} \in\left(\begin{array}{c}V \\ 2\end{array}\right)$, we put $\{u, v\} \in \hat{E}$ if, and only if,

$$
\{y\} \times U_{0} \not \supset(u, v) \notin \bigcup_{i \in \mathbb{Z}_{3}}\left(U_{i} \times U_{i}\right) .
$$

In other words, $G=(V, E)$ and $\hat{G}=(V, \hat{E})$ differ only in the $3 m=n-2$ elements among

$$
\hat{E} \backslash E=\bigcup\left\{\left\{x, u_{0}\right\}: u_{0} \in U_{0}\right\} \cup \bigcup\left\{\left\{y, u_{1}\right\}: u_{1} \in U_{1}\right\} \quad \text { and } \quad E \backslash \hat{E}=\bigcup\left\{\left\{y, v_{0}\right\}: v_{0} \in U_{0}\right\} .
$$

Define $\hat{c}: \hat{E} \rightarrow\{\star\} \cup V$ by setting, for each $e \in \hat{E}$,

$$
\hat{c}(e)=\left\{\begin{array}{cc}
\star & \text { if } e \in \hat{E} \backslash E, \\
\star & \text { if } e=\left\{x, u_{2}\right\} \in \hat{E} \cap E \text { for some } u_{2} \in U_{2}, \\
c_{+}(e) & \text { otherwise. }
\end{array}\right.
$$

We observe from (5D) that $(\hat{G}, \hat{c})$ is $(m+2)$-color-regular, while $\lceil(n+5) / 3)\rceil=m+3$ is ensured by $n \equiv 2(\bmod 3)$. Indeed, fix a vertex $u_{0} \in U_{0}$, and fix an incident edge $u_{0} \in e \in \hat{E}$. If $x \in e$, then $e$ is assigned the fixed color $\hat{c}(e)=\star$, and if $e \cap U_{2} \neq \emptyset$, then $e$ is assigned the fixed color $\hat{c}(e)=c_{+}(e)=u_{0}$. Otherwise, $e=\left\{u_{0}, u_{1}\right\} \in \hat{E}\left[U_{0}, U_{1}\right]=E\left[U_{0}, U_{1}\right]$ for some $u_{1} \in U_{1}$, whence $e$ is assigned the variable color $\hat{c}(e)=c_{+}(e)=u_{1}$ among all $\left|U_{1}\right|=m$ many possible $u_{1} \in U_{1}$. Vertices $u_{1} \in U_{1}$ and $u_{2} \in U_{2}$ similarly have $\hat{c}$-degree $m+2$. For the fixed vertex $x \in V$, fix an incident edge $x \in e \in \hat{E}$. If $e \cap\left(U_{0} \cup U_{2}\right) \neq \emptyset$, then 
$e$ is assigned the fixed color $\hat{c}(e)=\star$, and if $y \in e$, then $e$ is assigned the fixed color $\hat{c}(e)=y$. Otherwise, $e=\left\{x, u_{1}\right\}$ for some $u_{1} \in U_{1}$, whence $e$ is assigned the variable color $\hat{c}(e)=c_{+}(e)=u_{1}$ among all $\left|U_{1}\right|=m$ many possible $u_{1} \in U_{1}$. The fixed vertex $y \in V$ similarly has $\hat{c}$-degree $m+2$. That $(\hat{G}, \hat{c})$ avoids rainbow $\ell$-cycles $C_{\ell}$ is sketched in the Appendix, when more needed concepts are developed.

\section{Proofs of Theorems 1.2 and 1.4}

The proofs of Theorems 1.2 and 1.4 are based on the well-known stability method, together with a few elementary results. We present the tools we need in order of increasing technicality.

2.1. Elementary tools. Edge-colored graphs $(G, c)$ on a vertex set $V$ correspond to directed graphs $\vec{G}=(V, \vec{E})$, as follows. For each $v \in V$, let $\left\{v, w_{1}\right\}, \ldots,\left\{v, w_{d}\right\} \in E$ be a system of representatives of the color classes of $c$ on edges at $v$, where $d=\operatorname{deg}_{G}^{c}(v)$. We put $\left(v, w_{1}\right), \ldots,\left(v, w_{d}\right) \in \vec{E}$, and we say that a directed graph $\vec{G}=(V, \vec{E})$ obtained in this way (which need be neither oriented nor unique) is associated with $(G, c)$. Directed graphs $\vec{G}=(V, \vec{E})$ correspond to edge-colored graphs $(G, c)$, as follows. For each $(v, w) \in \vec{E}$, we put $\{v, w\} \in E(G)$ and define $c(\{v, w\})=w$. Then $(G, c)$ is uniquely determined by $\vec{G}$, although $G=(V, E)$ may be a multigraph. We pause for the following remark.

Remark 2.1. In this paper, no directed graph $\vec{G}=(V, \vec{E})$ will allow $\vec{E}$ to be a multiset, nor will $\vec{E}$ consist of any directed loops. When $(v, w) \in \vec{E}$ forbids $(w, v) \in \vec{E}$, then $\vec{G}=(V, \vec{E})$ is an oriented graph. When so, the edge-colored graph $(G, c)$ determined by $\vec{G}$ is simple.

In the contexts above, we make a couple of elementary observations. On the one hand, if $(G, c)$ is an edge-colored graph and $\vec{G}=(V, \vec{E})$ is a directed graph associated with $(G, c)$, then every vertex $v \in V$ has out-degree $\operatorname{deg}_{\vec{G}}^{+}(v)=\operatorname{deg}_{G}^{c}(v)$. On the other hand, if $\vec{G}=(V, \vec{E})$ is an oriented graph and $(G, c)$ is the edge-colored graph determined by $\vec{G}=(V, \vec{E})$, then every vertex $v \in V$ satisfies $\operatorname{deg}_{G}^{c}(v)=\operatorname{deg}_{\vec{G}}^{+}(v)+1$ when $v$ has positive in-degree in $\vec{G}$, and $\operatorname{deg}_{G}^{c}(v)=\operatorname{deg}_{\vec{G}}^{+}(v)$ otherwise. In these contexts, we next consider the extent to which rainbow cycles of $(G, c)$ relate to directed cycles of $\vec{G}$, and vice versa. We begin with the following elementary but useful observation first noted by $\mathrm{H}$. Li in [16].

Fact 2.2. Let $\vec{G}=(V, \vec{E})$ be an oriented graph, and let $(G, c)$ be the edge-colored graph determined by $\vec{G}$. Every directed $\ell$-cycle $\vec{C}_{\ell}$ in $\vec{G}$ corresponds to a rainbow $\ell$-cycle $C_{\ell}$ in $(G, c)$. Moreover, every properly colored $\ell$-cycle $C_{\ell}$ in $(G, c)$ is, in fact, a rainbow $\ell$-cycle, and corresponds to a directed $\ell$-cycle $\vec{C}_{\ell}$ in $\vec{G}$.

In Fact 2.2, the edge-colored graph $(G, c)$ is derived from a given oriented graph $\vec{G}=(V, \vec{E})$, and directed $\ell$-cycles $\vec{C}_{\ell}$ of $\vec{G}$ are in one-to-one correspondence with rainbow $\ell$-cycles $C_{\ell}$ of $(G, c)$. However, when $(G, c)$ is given and $\vec{G}=(V, \vec{E})$ is associated with $(G, c)$, the same conclusion need not hold.

Fact 2.3. Let $(G, c)$ be an n-vertex edge-colored graph, and let $\vec{G}=(V, \vec{E})$ be a directed graph associated with $(G, c)$. Then $\vec{G}$ admits at most $n^{\ell-1}$ many directed $\ell$-cycles $\vec{C}_{\ell}$ that were not rainbow in $(G, c)$.

Proof of Fact 2.3. Let $\mathcal{A}_{\ell}\left(\mathcal{N}_{\ell}\right)$ denote the family of all (non-rainbow) directed $\ell$-cycles $\left(v_{0}, v_{1}, \ldots, v_{\ell-1}\right)$ in $\vec{G}$, written here as cyclic permutations. Then

$$
\ell\left|\mathcal{A}_{\ell}\right|=\sum_{v_{0} \in V} \sum_{v_{1} \in N_{\vec{G}}^{+}\left(v_{0}\right)} \ldots \sum_{v_{\ell-2} \in N_{\vec{G}}^{+}\left(v_{\ell-3}\right)} \sum_{v_{\ell-1} \in N_{\vec{G}}^{+}\left(v_{\ell-2}\right) \cap N_{\vec{G}}^{-}\left(v_{0}\right)} 1 .
$$

Each $\left(v_{0}, v_{1}, \ldots, v_{\ell-1}\right) \in \mathcal{N}_{\ell}$ identifies a sum in (6) with at most $\ell$-terms, so $\ell\left|\mathcal{N}_{\ell}\right| \leq \ell n^{\ell-1}$.

The following concept is central throughout the remainder of the paper.

Definition 2.4 ( $\lambda$-extremal). Fix $\lambda \geq 0$, an $n$-vertex directed graph $\vec{G}=(V, \vec{E})$, and an edge-colored graph $(G, c)$ with vertex set $V$ and edge set $E$. We say that 
(1) $\vec{G}$ is $\lambda$-extremal if there exists a partition $V=V_{0} \cup V_{1} \cup V_{2}$ where for all $i \in \mathbb{Z}_{3}$,

$$
e_{\vec{G}}\left(V_{i}, V_{i+1}\right) \geq\left(\frac{1}{9}-\lambda\right) n^{2},
$$

where $e_{\vec{G}}\left(V_{i}, V_{i+1}\right)$ denotes the number of edges $\left(v_{i}, v_{i+1}\right) \in \vec{E} \cap\left(V_{i} \times V_{i+1}\right)$;

(2) $(G, c)$ is $\lambda$-extremal if there exists a partition $V=V_{0} \cup V_{1} \cup V_{2}$ on which some directed graph $\vec{G}=(V, \vec{E})$ associated with $(G, c)$ is $\lambda$-extremal.

In these contexts, $V=V_{0} \cup V_{1} \cup V_{2}$ is said to be $\lambda$-extremal for $\vec{G}$ or $(G, c)$.

We conclude our elementary tools with the following fact.

Fact 2.5. For all $0 \leq \lambda \leq 1 /(28)$, and for every positive integer $\ell \equiv 0(\bmod 3)$, the following hold:

(1) Every $\lambda$-extremal n-vertex directed graph $\vec{G}=(V, \vec{E})$ has $\Omega\left(n^{\ell}\right)$ many directed $\ell$-cycles $\vec{C}_{\ell}$.

(2) Every $\lambda$-extremal $n$-vertex edge-colored graph $(G, c)$ has $\Omega\left(n^{\ell}\right)$ many rainbow $\ell$-cycles $C_{\ell}$.

Proof of Fact 2.5. Fix $0 \leq \lambda \leq 1 /(28)$ and fix a positive integer $\ell \equiv 0(\bmod 3)$. To prove Statement (1), set $k=\ell / 3$, and let $\vec{G}=(V, \vec{E})$ be an $n$-vertex directed graph with $\lambda$-extremal vertex partition $V=$ $V_{0} \cup V_{1} \cup V_{2}$. Let $\vec{H}$ be a blow-up of the directed triangle on $V=V_{0} \cup V_{1} \cup V_{2}$, whose edges consist of $\left(V_{0} \times V_{1}\right) \cup\left(V_{1} \times V_{2}\right) \cup\left(V_{2} \times V_{0}\right)$. Then, $\vec{H}$ admits precisely $\left(\left|V_{0}\right|\right)_{k} \times\left(\left|V_{1}\right|\right)_{k} \times\left(\left|V_{2}\right|\right)_{k}$ many directed $\ell$-cycles $\vec{C}_{\ell}$ meeting each of $V_{0}, V_{1}$, and $V_{2}$ exactly $k$ times. The number of these cycles having some edge $\vec{e}=\left(v_{0}, v_{1}\right)$ of $\vec{H} \backslash \vec{G}$, where $v_{0} \in V_{0}$ and $v_{1} \in V_{1}$, is at most

$$
\left(\left|V_{0}\right|\left|V_{1}\right|-\left(\frac{1}{9}-\gamma\right) n^{2}\right)\left|V_{2}\right| \times\left(\left|V_{0}\right|-1\right)_{k-1} \times\left(\left|V_{1}\right|-1\right)_{k-1}\left(\left|V_{2}\right|-1\right)_{k-1} .
$$

More generally, the number of these cycles having some edge $\vec{e}$ of $\vec{H} \backslash \vec{G}$ is at most

$$
\left(3-\left(\frac{1}{9}-\gamma\right) \frac{n^{3}}{\left|V_{0}\right|\left|V_{1}\right|\left|V_{2}\right|}\right)\left(\left|V_{0}\right|\right)_{k} \times\left(\left|V_{1}\right|\right)_{k} \times\left(\left|V_{2}\right|\right)_{k} .
$$

Thus, $\vec{G}$ admits at least

$$
\left(\left(\frac{1}{9}-\gamma\right) \frac{n^{3}}{\left|V_{0}\right|\left|V_{1}\right|\left|V_{2}\right|}-2\right)\left(\left|V_{0}\right|\right)_{k} \times\left(\left|V_{1}\right|\right)_{k} \times\left(\left|V_{2}\right|\right)_{k}
$$

many directed $\ell$-cycles $\vec{C}_{\ell}$. Since $\left|V_{0}\right|\left|V_{1}\right|\left|V_{2}\right| \leq n^{3} /(27)$ holds by convexity, $\vec{G}$ admits at least

$$
(1-27 \gamma)\left(\left|V_{0}\right|\right)_{k} \times\left(\left|V_{1}\right|\right)_{k} \times\left(\left|V_{2}\right|\right)_{k}=\Omega\left(n^{\ell}\right)
$$

many directed $\ell$-cycles $\vec{C}_{\ell}$, where we used $\gamma \leq 1 /(28)$.

For Statement $(2)$, let $(G, c)$ be an $n$-vertex $\lambda$-extremal edge colored graph, and let $\vec{G}=(V, \vec{E})$ be a directed graph associated with $(G, c)$ which has $\lambda$-extremal partition $V=V_{0} \cup V_{1} \cup V_{2}$. Let $\vec{F} \subseteq \vec{G}$ consist of all $\left(v_{i}, v_{i+1}\right) \in \vec{E}$ where $v_{i} \in V_{i}$ and $v_{i+1} \in V_{i+1}$ for $i \in \mathbb{Z}_{3}$. Then $\vec{F}$ is an oriented graph with $\lambda$-extremal partition $V=V_{0} \cup V_{1} \cup V_{2}$ which, by Statement (1), admits $\Omega\left(n^{\ell}\right)$ directed $\ell$-cycles $\vec{C}_{\ell}$. Fact 2.3 ensures that $\Omega\left(n^{\ell}\right)-n^{\ell-1}$ of these directed cycles correspond to rainbow cycles in $(G, c)$, because the edge-colored graph $F$ determined by $\vec{F}$ is, by construction, a subgraph of $G$.

2.2. Stability results. In what follows, we distinguish between whether or not a given structure is $\lambda$-extremal (cf. Definition 2.4).

Lemma 2.6. For all $\lambda>0$, there exists $\alpha=\alpha(\lambda)>0$ so that for all integers $\ell \geq 4$, there exists an integer $n_{0}=n_{0}(\lambda, \alpha, \ell) \geq 1$ so that whenever $\vec{G}$ is an oriented graph on $n \geq n_{0}$ many vertices satisfying

$$
\delta^{+}(\vec{G}) \geq\left\{\begin{array}{cl}
\left(\frac{1}{3}-\alpha\right) n & \text { if } \ell \neq 5, \\
\frac{n+1}{3} & \text { if } \ell=5,
\end{array}\right.
$$

then $\vec{G}$ is $\lambda$-extremal or $\vec{G}$ admits a closed directed $\ell$-walk $\vec{W}_{\ell}$.

We prove Lemma 2.6 in Sections 34, We apply Lemma 2.6 in the following convenient form. 
Corollary 2.7 (the non-extremal case). In the context of Lemma 2.6, the following statements hold:

(1) If $\ell=5$ and $\vec{G}$ is not $\lambda$-extremal, then $\vec{G}$ contains a directed 5-cycle $\vec{C}_{5}$;

(2) If $\ell \neq 5$ and $\vec{G}$ is not $\lambda$-extremal, then $\vec{G}$ contains $\Omega\left(n^{\ell}\right)$ many directed $\ell$-cycles $\vec{C}_{\ell}$.

Moreover, for even integers $\ell$, Statement (2) above holds when $\vec{G}$ is allowed to be a directed graph.

Note that Statement (1) of Corollary 2.7 restates the conclusion of Lemma 2.6 when $\ell=5$, since the only closed directed 5 -walk $\vec{W}_{5}$ is the 5 -cycle $\vec{C}_{5}$. It is standard to derive Statement (2) of Corollary 2.7 from Lemma [2.6] by using a suitable regularity lemma. We sketch such a proof below.

Remark 2.8. In the context of Lemma 2.6, let $\vec{G}$ be an oriented graph on $n \geq n_{0}(\lambda, \ell)$ many vertices which satisfies (8), where $\ell \neq 5$. We may apply Lemma 3.2 from [11] to obtain a regular partition $V=V_{0} \cup V_{1} \cup \cdots \cup V_{t}$ of $\vec{G}$ with cluster digraph $\vec{R}$, where $\vec{R}$ may not be oriented. Nonetheless, Lemma 3.2 guarantees that $\vec{R}$ admits an oriented spanning subgraph $\vec{Q} \subseteq \vec{R}$, where $\delta^{+}(\vec{Q}) / t$ can be taken arbitrarily close to $\delta^{+}(\vec{G}) / n$, and where $\delta^{-}(\vec{Q}) / t$ can be taken arbitrarily close to $\delta^{-}(\vec{G}) / n$. As such, if the oriented graph $\vec{G}$ is not $\lambda$-extremal, then the oriented graph $\vec{Q}$ isn't $\lambda^{\prime}$-extremal for some suitably small $0<\lambda^{\prime} \leq \lambda$. Lemma 2.6 then guarantees that $\vec{Q}$ admits a closed directed $\ell$-walk $\vec{W}_{\ell}$. Applying a counting lemma to the system of pairs $\left(V_{i}, V_{j}\right)$ corresponding to the edges of $\vec{W}_{\ell}$ guarantees $\Omega\left(n^{\ell}\right)$ many directed $\ell$-cycles $\vec{C}_{\ell}$.

When $\ell$ is even, $\vec{G}$ need not be oriented. Here, we may apply Lemma 3.1 of [2] to obtain a regular partition $V=V_{0} \cup V_{1} \cup \cdots \cup V_{t}$ of $\vec{G}$ with cluster digraph $\vec{R}$. Again, if $\vec{G}$ is not $\lambda$-extremal, then $\vec{R}$ is not $\lambda^{\prime}$-extremal for some suitably small $0<\lambda^{\prime} \leq \lambda$. If $\vec{R}$ is, in fact, an oriented graph, then we proceed identically to the above. Assume that $\vec{R}$ admits a 2-cycle, i.e., a closed 2-walk $\vec{W}_{2}$. Since $\ell$ is even, the pair $\left(V_{i}, V_{j}\right)$ corresponding to $\vec{W}_{2}$ admits $\Omega\left(n^{\ell}\right)$ many directed $\ell$-cycles $\vec{C}_{\ell}$.

We continue with an extremal counterpart to Corollary 2.7.

Lemma 2.9 (the extremal case). There exists an absolute constant $\lambda_{0}>0$ so that, for all $0<\lambda \leq \lambda_{0}$ and for all integers $\ell \geq 4$ not divisible by three, there exists an integer $n_{0}=n_{0}\left(\lambda_{0}, \lambda, \ell\right) \geq 1$ so that whenever $(G, c)$ is a $\lambda$-extremal edge colored graph on $n \geq n_{0}$ many vertices, the following hold:

(1) If $\ell \neq 5$ and $\delta^{c}(G) \geq(n+5) / 3$ (cf. (10), then $(G, c)$ admits a rainbow $\ell$-cycle $C_{\ell}$;

(2) If $\delta^{c}(G) \geq(n+4) / 3$, then $(G, c)$ admits a properly colored $\ell$-cycle $C_{\ell}$.

We prove Lemma 2.9 in Sections 6, 8 . We proceed to the proofs of Theorems 1.2 and 1.4 which are formal consequences of Corollary 2.7 and Lemma 2.9.

2.3. Proof of Theorem 1.2. To define the absolute constant $\alpha>0$ promised by Theorem 1.2, we consider auxiliary parameters. Let $\lambda_{\text {Lem } 2.9}$ be the absolute constant $\lambda_{0}$ guaranteed by Lemma 2.9. Set

$$
\lambda=\min \left\{\frac{1}{28}, \lambda_{\text {Lem } 2.9}\right\},
$$

which is suitably small for an application of Fact 2.5. With $\lambda>0$ given in (9), let

$$
\alpha=\alpha_{\text {Lem } 2.6}(\lambda)>0
$$

be the constant guaranteed by Lemma 2.6, which we take to be the constant promised by Theorem 1.2 .

Fix an even integer $\ell \geq 4$. Let $(G, c)$ be an $n$-vertex edge-colored graph satisfying (1), where in all that follows we assume that $n \geq n_{0}(\lambda, \alpha, \ell)$ is sufficiently large. To prove Theorem 1.2, we distinguish between the cases of whether or not $(G, c)$ is $\lambda$-extremal, where $\lambda$ is given in (9).

Case $1((G, c)$ is $\lambda$-extremal). In this case, we apply Fact 2.5 or Lemma2.9 to $(G, c)$. Assume first that $\ell \equiv 0(\bmod 3)$. By our choice of $\lambda \leq 1 /(28)$ from (9), Statement $(2)$ of Fact 2.5 guarantees $\Omega\left(n^{\ell}\right)$ many rainbow $\ell$-cycles $C_{\ell}$ in $(G, c)$. Assume now that $\ell \not \equiv 0(\bmod 3)$. By our choice of $\lambda \leq \lambda_{\text {Lem } 2.9 \text { from (9), }}$, Statement (1) of Lemma 2.9 guarantees a rainbow $\ell$-cycle $C_{\ell}$ in $(G, c)$. (Note: $\ell \neq 5$ by the parity of $\ell$.) 
Case $2((G, c)$ is not $\lambda$-extremal). In this case, we will indirectly apply Fact 2.3 and Corollary 2.7 to $(G, c)$. For that, let $\vec{G}=(V, \vec{E})$ be any directed graph associated with $(G, c)$, where necessarily $\vec{G}$ is not $\lambda$-extremal, and where $\delta^{+}(\vec{G})=\delta^{c}(G) \geq\left(\frac{1}{3}-\alpha\right) n$ is ensured by (11). By our choice of $\alpha=\alpha_{\text {Lem [2.6 }}(\lambda)$ in (10) (and $\ell \neq 5$ ), Statement (2) of Corollary 2.7 guarantees $\Omega\left(n^{\ell}\right)$ many directed $\ell$-cycles $\vec{C}_{\ell}$ in $\vec{G}$. Fact 2.3 then guarantees that at least one of these corresponds to a rainbow $\ell$-cycle $C_{\ell}$ in $(G, c)$.

2.4. Proof of Theorem 1.4. We again use the auxiliary constants $\lambda>0$ and $\alpha>0$ determined in (9) and (10). Fix an integer $\ell \geq 4$. Let $\vec{G}=(V, \vec{E})$ be an $n$-vertex oriented graph satisfying $\delta^{+}(\vec{G}) \geq(n+1) / 3$, where in all that follows we assume that $n \geq n_{0}(\lambda, \alpha, \ell)$ is sufficiently large. Let $\vec{H} \subseteq \vec{G}$ be maximally induced w.r.t. satisfying $\delta^{-}(\vec{H}) \geq 1$, and set $U=V(\vec{H})$. Note that every $u \in U$ satisfies $\operatorname{deg}_{\vec{H}}^{+}(u)=\operatorname{deg}_{\vec{G}}^{+}(u)$. Consequently, $|U|=\Omega(n)$ can be taken as large as needed since the number $e(\vec{H})$ of edges of $\vec{H}$ satisfies

$$
\left(\begin{array}{c}
|U| \\
2
\end{array}\right) \geq e(\vec{H}) \geq|U| \delta^{+}(\vec{H}) \geq|U| \delta^{+}(\vec{G}) \quad \Longrightarrow \quad|U| \geq 2 \delta^{+}(\vec{G}) \geq 2 n / 3 .
$$

We now distinguish between the cases of whether or not $\vec{H}$ is $\lambda$-extremal, where $\lambda$ is determined in (9).

Case 1 ( $\vec{H}$ is not $\lambda$-extremal). In this case, we apply Corollary 2.7 to $\vec{H}$, which is possible on account that $\delta^{+}(\vec{H}) \geq \delta^{+}(\vec{G}) \geq(n+1) / 3 \geq((1 / 3)-\alpha) n$, for $\alpha=\alpha_{\text {Lem } 2.7}$ in (10). Whether or not $\ell=5$, Corollary 2.7 guarantees a directed $\ell$-cycle $\vec{C}_{\ell}$ in $\vec{H}$, where $\vec{C}_{\ell}$ also appears in $\vec{G} \supseteq \vec{H}$.

Case $2(\vec{H}$ is $\lambda$-extremal). In this case, we will apply Fact 2.5 to $\vec{H}$ or we will indirectly apply Fact 2.2 and Lemma 2.9 to $\vec{H}$. Assume first that $\ell \equiv 0(\bmod 3)$. By our choice of $\lambda \leq 1 /(28)$ in (9), Statement (1) of Fact 2.5 guarantees $\Omega\left(n^{\ell}\right)$ many directed $\ell$-cycles $\vec{C}_{\ell}$ in $\vec{H}$, each of which also appears in $\vec{G} \supseteq \vec{H}$. Assume now that $\ell \not \equiv 0(\bmod 3)$. Let $(H, c)$ be the edge-colored graph determined by $\vec{H}$, where $H$ has vertex set $U=V(\vec{H})$. Since every vertex $u \in U$ has positive in-degree in $\vec{H}$, we have that

$$
\operatorname{deg}_{H}^{c}(u)=1+\operatorname{deg}_{\vec{H}}^{+}(u) \geq \frac{n+4}{3} .
$$

By our choice of $\lambda \leq \lambda_{\text {Lem [2.9] }}$ in (9), Statement (2) of Lemma 2.9) guarantees a properly colored $\ell$-cycle $C_{\ell}$ in $(H, c)$. Since $(H, c)$ was determined by the oriented graph $\vec{H}$, Fact 2.2 guarantees that $C_{\ell}$ corresponds to a directed $\ell$-cycle $\vec{C}_{\ell}$ in $\vec{H}$, which also appears in $\vec{G} \supseteq \vec{H}$.

\section{Proof of Lemma 2.6}

Lemma 2.6 is a formal consequence of the following two propositions (recall $\delta_{0}(\vec{G})$ from Theorem 1.5).

Proposition 3.1. For all $\beta>0$, there exists $\alpha=\alpha(\beta)>0$ so that for every integer $\ell \geq 4$, there exists an integer $n_{0}=n_{0}(\beta, \alpha, \ell) \geq 1$ so that the following holds. Let $\vec{G}=(V, \vec{E})$ be an oriented graph satisfying (8) on $n \geq n_{0}$ many vertices. If $\vec{G}$ admits no closed directed $\ell$-walk, then $\vec{G}$ admits an induced subgraph $\vec{H}=\vec{G}[U]$ on $|U|=m \geq(1-\beta) n$ many vertices which satisfies

$$
\delta_{0}(\vec{H}) \geq\left(\frac{\delta^{+}(\vec{G})}{n}-\beta\right) m .
$$

Proposition 3.2. For all $\lambda_{0}>0$, there exists $\beta=\beta\left(\lambda_{0}\right)>0$ so that for every integer $\ell \geq 4$, there exists an integer $m_{0}=m_{0}\left(\lambda_{0}, \beta, \ell\right) \geq 1$ so that the following holds. Let $\vec{H}$ be an oriented graph on $m \geq m_{0}$ vertices which admits no closed directed $\ell$-walk, but which satisfies $\delta_{0}(\vec{H}) \geq((1 / 3)-\beta) m$. Then $\vec{H}$ is $\lambda_{0}$-extremal.

The proof of Proposition 3.1 is not too difficult, and will be given later in this section. The proof of Proposition 3.2 is more involved, and will be postponed to the following section. 
3.1. Proof of Lemma 2.6. Let $\lambda>0$ be given. To define the constant $\alpha=\alpha(\lambda)>0$ promised by Lemma 2.6 we consider several auxiliary constants. First, set $\lambda_{0}=\lambda / 2$, and let

$$
\beta_{\text {Prop } 3.2}=\beta_{\text {Prop } 3.2}\left(\lambda_{0}\right)>0
$$

be the constant guaranteed by Proposition 3.2 Second, set

$$
\beta=\frac{1}{2} \min \left\{\lambda_{0}, \beta_{\operatorname{Prop}[3.2}\right\} .
$$

Third, let

$$
\alpha_{\text {Prop } 3.1}=\alpha_{\text {Prop } 3.1}(\beta)>0
$$

be the constant guaranteed by Proposition 3.1 We define

$$
\alpha=\min \left\{\alpha_{\text {Prop } 3.1,}, \beta\right\}
$$

to be the constant promised by Lemma2.6. Let an integer $\ell \geq 4$ be given. Let $\vec{G}=(V, \vec{E})$ be an $n$-vertex oriented graph satisfying (8) with $\alpha$ in (15), where in all that follows we assume that $n \geq n_{0}(\lambda, \alpha, \ell)$ is sufficiently large. We assume that $\vec{G}$ admits no closed directed $\ell$-walk, and establish that $\vec{G}$ is $\lambda$-extremal.

Since $\vec{G}$ admits no closed directed $\ell$-walk, and by our choice of $\alpha \leq \alpha_{\operatorname{Prop} 3.1}$ in (14) and (15), Proposition 3.1 guarantees that $\vec{G}$ admits an induced subgraph $\vec{H}=\vec{G}[U]$ on $|U|=m \geq(1-\beta) n$ (cf. (13)) many vertices for which

$$
\delta_{0}(\vec{H}) \stackrel{\sqrt[11]{\geq}}{\geq}\left(\frac{\delta^{+}(\vec{G})}{n}-\beta\right) m \stackrel{\sqrt[8]{\geq}}{\geq}\left(\frac{1}{3}-\alpha-\beta\right) m \stackrel{\sqrt[15]{\geq}}{\geq}\left(\frac{1}{3}-2 \beta\right) m \stackrel{\sqrt[13]{\geq}}{\geq}\left(\frac{1}{3}-\beta_{\text {Prop (3.2) }}\right) m .
$$

Since $\vec{H}$ admits no closed directed $\ell$-walk, and by our choice of $\beta_{\text {Prop } 3.2}$ in (12), Proposition 3.2 guarantees that $\vec{H}$ is $\lambda_{0}$-extremal. Let $U=V(\vec{H})=U_{0} \cup U_{1} \cup U_{2}$ be any $\lambda_{0}$-extremal partition of $\vec{H}$ (cf. Definition 2.4), and let $V=V(\vec{G})=V_{0} \cup V_{1} \cup V_{2}$ be any partition satisfying $U_{i} \subseteq V_{i}$ for each $0 \leq i \leq 2$. Then, for each $i \in \mathbb{Z}_{3}$,

$$
\begin{aligned}
& e_{\vec{G}}\left(V_{i}, V_{i+1}\right) \geq e_{\vec{G}}\left(U_{i}, U_{i+1}\right)=e_{\vec{H}}\left(U_{i}, U_{i+1}\right) \\
& \quad \stackrel{\text { Prop } 3.2}{\geq}\left(\frac{1}{9}-\lambda_{0}\right) m^{2} \stackrel{\text { Prop } 3.1}{\geq}\left(\frac{1}{9}-\lambda_{0}\right)(1-\beta)^{2} n^{2} \stackrel{13}{\geq}\left(\frac{1}{9}-\lambda\right) n^{2},
\end{aligned}
$$

where we also used $\lambda=2 \lambda_{0}$. Thus, $V=V_{0} \cup V_{1} \cup V_{2}$ is a $\lambda$-extremal partition of $\vec{G}$, as desired.

3.2. Proof of Proposition 3.1. Let $\beta>0$ be given. Define

$$
\alpha=\beta^{6} /(96) .
$$

Let integer $\ell \geq 4$ be given. Let $\vec{G}=(V, \vec{E})$ be an $n$-vertex oriented graph satisfying (8), where in all that follows, we take $n \geq n_{0}(\beta, \alpha, \ell)$ to be sufficiently large. Assume that $\vec{G}$ admits no closed directed $\ell$-walks. The subgraph $\vec{H}=\vec{G}[U]$ desired in (11) is induced on the following vertices of large in-degree:

$$
U=V_{\text {high }}=\left\{v \in V: \operatorname{deg}_{\vec{G}}^{-}(v) \geq \delta^{+}(\vec{G})-n\left(\beta^{2} / 2\right)\right\} .
$$

To see that $\vec{H}=\vec{G}\left[V_{\text {high }}\right]$ satisfies (11), we use the following claim (whose proof we defer for a moment).

Claim 3.3. $\Delta^{-}(\vec{G}) \leq \delta^{+}(\vec{G})+n\left(\beta^{3} / 4\right)$, where $\Delta^{-}(\vec{G})$ denotes the maximum in-degree in $\vec{G}$.

Using Claim 3.3 we will verify that $|U|=\left|V_{\text {high }}\right|=m \geq(1-\beta) n$. Indeed, with $V_{\text {low }}=V \backslash V_{\text {high }}$,

$$
\begin{aligned}
& n \delta^{+}(\vec{G}) \leq \sum_{u \in V} \operatorname{deg}_{\vec{G}}^{+}(u)=\sum_{v \in V} \operatorname{deg}_{\vec{G}}^{-}(v)=\sum_{w \in V_{\text {low }}} \operatorname{deg}_{\vec{G}}^{-}(v)+\sum_{x \in V_{\text {high }}} \operatorname{deg}_{\vec{G}}^{-}(w)
\end{aligned}
$$

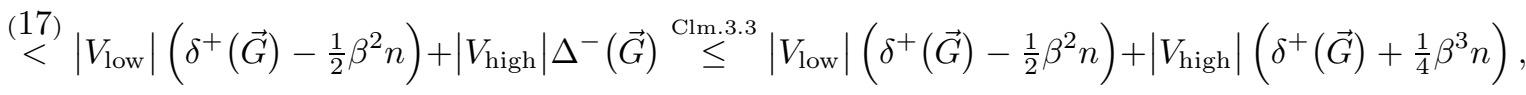


from which $2\left|V_{\text {low }}\right| \leq \beta\left|V_{\text {high }}\right| \leq \beta n$ and $\left|V_{\text {high }}\right| \geq(1-(\beta / 2)) n$ follow. By construction, both

$$
\begin{aligned}
& \delta^{+}(\vec{H}) \geq \delta^{+}(\vec{G})-\left|V_{\text {low }}\right| \geq \delta^{+}(\vec{G})-\frac{1}{2} \beta n \geq\left(\frac{\delta^{+}(\vec{G})}{n}-\beta\right) n \geq\left(\frac{\delta^{+}(\vec{G})}{n}-\beta\right) m, \\
& \quad \text { and } \quad \delta^{-}(\vec{H}) \geq \min \left\{\operatorname{deg}_{\vec{G}}^{-}(v): v \in V_{\text {high }}\right\}-\left|V_{\text {low }}\right| \frac{17}{\geq} \delta^{+}(\vec{G})-\frac{1}{2} \beta^{2} n-\frac{1}{2} \beta n \geq\left(\frac{\delta^{+}(\vec{G})}{n}-\beta\right) m
\end{aligned}
$$

hold, as promised in (11). Thus, it remains to prove Claim 3.3] where we will use the following fact.

Fact 3.4. Let $R, S \subset V$ be some disjoint pair with sizes $|R| \geq \Delta^{-}(\vec{G})$ and $|S| \geq \delta^{+}(\vec{G})$, where $(S, R)$ admits no path $s \rightarrow v \rightarrow r$ in $\vec{G}$ with $s \in S$ and $r \in R$. Then $\Delta^{-}(\vec{G}) \leq \delta^{+}(\vec{G})+n\left(\beta^{3} / 4\right)$ (cf. Claim [3.3).

Proof of Fact 3.4. Let $R, S \subset V$ be given as above. Fix $S_{0} \subseteq S$ with $\left|S_{0}\right|=\delta^{+}(\vec{G})$ and set $S_{1}=N_{\vec{G}}^{+}\left(S_{0}\right)$. Then $N_{\vec{G}}^{+}\left(S_{1}\right) \cap R=\emptyset$. Set $S_{2}=N_{\vec{G}}^{+}\left(S_{1}\right) \backslash S_{0}$ so that $R, S_{0}$ and $S_{2}$ are pairwise disjoint. Thus

$$
\Delta^{-}(\vec{G})+\delta^{+}(\vec{G})+\left|S_{2}\right| \leq|R|+\left|S_{0}\right|+\left|S_{2}\right| \leq n .
$$

We double-count the number $e_{\vec{G}}\left(S_{1}, S_{2}\right)$ of edges from $S_{1}$ to $S_{2}$. On the one hand,

$$
e_{\vec{G}}\left(S_{1}, S_{2}\right) \leq\left|S_{2}\right| \Delta^{-}(\vec{G}) \stackrel{18}{\leq} \Delta^{-}(\vec{G})\left(n-\Delta^{-}(\vec{G})-\delta^{+}(\vec{G})\right) .
$$

On the other hand,

$$
\begin{aligned}
e_{\vec{G}}\left(S_{1}, S_{2}\right) \geq\left|S_{1}\right| \delta^{+}(\vec{G})-e_{\vec{G}}\left(S_{1}, S_{0}\right) \geq\left|S_{1}\right| \delta^{+}(\vec{G})- & \left(\left|S_{0}\right|\left|S_{1}\right|-e_{\vec{G}}\left(S_{0}, S_{1}\right)\right) \\
& =e_{\vec{G}}\left(S_{0}, S_{1}\right) \geq\left|S_{0}\right| \delta^{+}(\vec{G})=\left(\delta^{+}(\vec{G})\right)^{2},
\end{aligned}
$$

where we twice used that $\left|S_{0}\right|=\delta^{+}(\vec{G})$. Comparing (19) and (20), we infer

$$
\begin{aligned}
& \left(\Delta^{-}(\vec{G})\right)^{2}-\left(n-\delta^{+}(\vec{G})\right) \Delta^{-}(\vec{G})+\left(\delta^{+}(\vec{G})\right)^{2} \leq 0 \\
& \Longrightarrow \quad \Delta^{-}(\vec{G}) \leq \frac{1}{2}\left(n-\delta^{+}(\vec{G})+\sqrt{\left(n-3 \delta^{+}(\vec{G})\right)\left(n+\delta^{+}(\vec{G})\right)}\right) \stackrel{(8)}{\leq} \frac{1}{2}\left(n-\delta^{+}(\vec{G})+n \sqrt{6 \alpha}\right) \\
& \quad=\delta^{+}(\vec{G})+\frac{1}{2}\left(n-3 \delta^{+}(\vec{G})+n \sqrt{6 \alpha}\right) \stackrel{8}{\leq} \delta^{+}(\vec{G})+\frac{1}{2} n(3 \alpha+\sqrt{6 \alpha}) \stackrel{\sqrt{16}}{\leq} \delta^{+}(\vec{G})+n \sqrt{6 \alpha},
\end{aligned}
$$

and so our choice of $\alpha=\beta^{6} /(96)$ from (16) completes the proof of Fact 3.4 .

We now prove Claim 3.3

Proof of Claim [3.3. Assume, on the contrary, that

$$
\Delta^{-}(\vec{G})>\delta^{+}(\vec{G})+\frac{1}{4} \beta^{3} n
$$

Then Fact 3.4 ensures that

every disjoint pair $R, S \subset V$ with $|R| \geq \Delta^{-}(\vec{G})$ and $|S| \geq \delta^{+}(\vec{G})$

$$
\text { admits a directed path } s \rightarrow v \rightarrow r \text { in } \vec{G} \text { with } s \in S \text { and } r \in R \text {. }
$$

Fix $x_{\max } \in V$ satisfying $\operatorname{deg}_{\vec{G}}^{-}\left(x_{\max }\right)=\Delta^{-}(\vec{G})$. We distinguish several cases of $\ell \geq 4$.

Case $1(\ell=4)$. Set $R=N_{\vec{G}}^{-}\left(x_{\max }\right)$ and $S=N_{\vec{G}}^{+}\left(x_{\max }\right)$, which are disjoint and satisfy $|R|=\Delta^{-}(\vec{G})$ and $|S| \geq \delta^{+}(\vec{G})$. Then (22) guarantees a directed 4-cycle $\left(x_{\max }, s, v, r, x_{\max }\right)$, which contradicts that $\vec{G}$ admits no closed directed 4 -walks. In other words, (21) must be false when $\ell=4$.

Case $2(\ell=5)$. We use the following peculiar observation, proven in a moment:

$$
\text { if } x_{\max } \rightarrow y \rightarrow z \rightarrow a \text { is a directed path in } \vec{G} \text {, then }\left(x_{\max }, a\right) \notin \vec{E},\left(x_{\max }, z\right) \notin \vec{E} \text {, and }(y, a) \notin \vec{E} \text {. }
$$


Using (23),$N_{\vec{G}}^{+}\left(x_{\max }\right)$ is an independent set whose every fixed element $y \in N_{\vec{G}}^{+}\left(x_{\max }\right)$ has an independent out-neighborhood $N_{\vec{G}}^{+}(y)$ which is disjoint from $N_{\vec{G}}^{+}\left(x_{\max }\right)$. Thus, for $z \in N_{\vec{G}}^{+}(y)$ fixed, it must be that $N_{\vec{G}}^{+}(z) \cap N_{\vec{G}}^{+}\left(x_{\max }\right) \neq \emptyset$ since otherwise $N_{\vec{G}}^{+}\left(x_{\max }\right) \cup N_{\vec{G}}^{+}(y) \cup N_{\vec{G}}^{+}(z) \subseteq V$ is a disjoint union with

$$
\operatorname{deg}_{\vec{G}}^{+}\left(x_{\max }\right)+\operatorname{deg}_{\vec{G}}^{+}(y)+\operatorname{deg}_{\vec{G}}^{+}(z) \geq 3 \delta^{+}(\vec{G}) \geq n+1 \quad(\text { recall } \ell=5 \text { in (8) }) .
$$

On the other hand, $N_{\vec{G}}^{+}(z) \cap N_{\vec{G}}^{+}\left(x_{\max }\right) \neq \emptyset$ violates (23), and so (21) is false when $\ell=5$.

To see (23), we first observe that

$$
N_{\vec{G}}^{+}(a) \cap N_{\vec{G}}^{-}\left(x_{\max }\right)=\emptyset,
$$

since $b \in N_{\vec{G}}^{+}(a) \cap N_{\vec{G}}^{-}\left(x_{\max }\right)$ would give the directed 5-cycle $\left(x_{\max }, y, z, a, b, x_{\max }\right)$, which would contradict that $\vec{G}$ admits no closed directed 5 -walks. Now, (24) forbids $\left(x_{\max }, a\right) \in \vec{E}$, since otherwise we set $R=N_{\vec{G}}^{-}\left(x_{\max }\right)$ and $S=N_{\vec{G}}^{+}(a)$ and use (22) to guarantee a directed 5-cycle $\left(x_{\max }, a, s, v, r, x_{\max }\right)$. We next observe that

$$
N_{\vec{G}}^{+}(a) \cap N_{\vec{G}}^{+}\left(x_{\max }\right) \neq \emptyset,
$$

since otherwise (24) gives that $N_{\vec{G}}^{+}(a) \cup N_{\vec{G}}^{+}\left(x_{\max }\right) \cup N_{\vec{G}}^{-}\left(x_{\max }\right) \subseteq V$ is a disjoint union with

$$
\operatorname{deg}_{\vec{G}}^{+}(a)+\operatorname{deg}_{\vec{G}}^{+}\left(x_{\max }\right)+\operatorname{deg}_{\vec{G}}^{-}\left(x_{\max }\right) \stackrel{\sqrt[21]{>}}{>} 3 \delta^{+}(\vec{G}) \stackrel{8}{\geq} n+1 .
$$

Using (25), fix $b \in N_{\vec{G}}^{+}(a) \cap N_{\vec{G}}^{+}\left(x_{\max }\right)$. Then

$$
N_{\vec{G}}^{+}(b) \cap N_{\vec{G}}^{-}\left(x_{\max }\right) \neq \emptyset,
$$

as otherwise we set $R=N_{\vec{G}}^{-}\left(x_{\max }\right)$ and $S=N_{\vec{G}}^{+}(b)$ and use (22) to guarantee a directed 5-cycle $\left(x_{\max }, b, s, v, r, x_{\max }\right)$. Using (26), we fix $c \in N_{\vec{G}}^{+}(b) \cap N_{\vec{G}}^{-}\left(x_{\max }\right)$, which forbids $\left(x_{\max }, z\right) \in \vec{E}$ lest $\left(x_{\max }, z, a, b, c, x_{\max }\right)$ is a directed 5-cycle. Similarly $(y, a) \notin \vec{E}$, which proves (23).

Case $3(\ell \geq 6)$. By the argument of Case $1, x_{\max }$ belongs to a directed 4-cycle $\vec{C}_{4}$. We first observe that $x_{\max }$ does not belong to a directed 3 -cycle $\vec{C}_{3}$. Indeed,

$$
\text { every integer } \ell \geq 6 \text { can be expressed as } \ell=3 i+4 j \text { for some integers } i, j \geq 0 \text {, }
$$

and so the inclusion of $x_{\max }$ along both a directed 3-cycle $\vec{C}_{3}$ and a directed 4-cycle $\vec{C}_{4}$ would place $x_{\max }$ in a closed directed $\ell$-walk in $\vec{G}$, contradicting our hypothesis. We next observe that a longest directed path $\vec{P}=\left(y_{1}, \ldots, y_{k}\right)$ in $N_{\vec{G}}^{+}\left(x_{\max }\right)$ satisfies $k=\Omega(n)$. Indeed, $\left|N_{\vec{G}}^{+}\left(y_{k}\right) \cap N_{\vec{G}}^{+}\left(x_{\max }\right)\right| \leq k-2$ holds by the optimal length of $\vec{P}$, and so

$$
\left|N_{\vec{G}}^{+}\left(y_{k}\right) \cup N_{\vec{G}}^{+}\left(x_{\max }\right)\right|=\operatorname{deg}_{\vec{G}}^{+}\left(y_{k}\right)+\operatorname{deg}_{\vec{G}}^{+}\left(x_{\max }\right)-\left|N_{\vec{G}}^{+}\left(y_{k}\right) \cap N_{\vec{G}}^{+}\left(x_{\max }\right)\right| \geq 2 \delta^{+}(\vec{G})-k .
$$

Since $x_{\text {max }}$ belongs to no directed 3 -cycles $\vec{C}_{3}$,

$$
\begin{array}{r}
N_{\vec{G}}^{+}\left(y_{k}\right) \cap N_{\vec{G}}^{-}\left(x_{\max }\right)=\emptyset=N_{\vec{G}}^{+}\left(x_{\max }\right) \cap N_{\vec{G}}^{-}\left(x_{\max }\right) \quad \Longrightarrow \quad N_{\vec{G}}^{+}\left(y_{k}\right) \cup N_{\vec{G}}^{+}\left(x_{\max }\right) \subseteq V \backslash N_{\vec{G}}^{-}\left(x_{\max }\right) \\
\Longrightarrow \quad\left|N_{\vec{G}}^{+}\left(y_{k}\right) \cup N_{\vec{G}}^{+}\left(x_{\max }\right)\right| \leq n-\operatorname{deg}_{\vec{G}}^{-}\left(x_{\max }\right)=n-\Delta^{-}(\vec{G}) .
\end{array}
$$

Then $k=\Omega(n)$ follows comparing (28) and (29):

$$
k \geq 2 \delta^{+}(\vec{G})+\Delta^{-}(\vec{G})-n \stackrel{21}{>} 3 \delta^{+}(\vec{G})+\frac{1}{4} \beta^{3} n-n \stackrel{8}{\geq} n\left(\frac{1}{4} \beta^{3}-3 \alpha\right) \stackrel{16}{=} n\left(\frac{1}{4} \beta^{3}-\frac{1}{32} \beta^{6}\right) \geq \beta^{3} n / 8 .
$$

To conclude Case 3 , set $R=N_{\vec{G}}^{-}\left(x_{\max }\right)$ and $S=N_{\vec{G}}^{+}\left(y_{k}\right)$, which we observed above are disjoint. Then (22) guarantees a path $(s, v, r)$ with $s \in S$ and $r \in R$, whence

$$
\left(x_{\max }, y_{k-\ell+5}, y_{k-\ell+6}, \ldots, y_{k}, s, v, r, x_{\max }\right)
$$

\footnotetext{
${ }^{1}$ This statement holds for all integers $\ell \geq 3$ outside of $\ell=5$, and can be proven by inducting on $\ell=\lfloor\ell / 2\rfloor+\lceil\ell / 2\rceil$.
} 
is a closed directed $\ell$-walk, contradicting our hypothesis. In other words, (21) must be false when $\ell \geq 6$. which proves Claim 3.3 .

\section{Proof of Proposition 3.2}

In this section, we prove Proposition 3.2 where we will use several auxiliary facts. The first fact is taken from Corollary 1.5 in 9 .

Fact 4.1. Fix an integer $\ell \geq 4$. Let $\vec{G}=(V, \vec{E})$ be a large $n$-vertex oriented graph which contains no directed triangle, but which satisfies $\delta_{0}(\vec{G}) \geq(0.3025) n$. Then $\vec{G}$ admits a directed $\ell$-cycle $\vec{C}_{\ell}$.

Our remaining facts are independent of the context of proving Proposition 3.2 and are therefore verified in Section 5 .

Fact 4.2. Fix an integer $\ell \geq 4$ and an $\varepsilon \in(0,1 /(11)]$. Let $\vec{G}=(V, \vec{E})$ be an oriented graph on $n \geq$ $n_{0}(\ell, \varepsilon)$ many vertices which admits no closed directed $\ell$-walk, but which satisfies $\delta_{0}(\vec{G}) \geq((1 / 3)-\varepsilon) n$. Let $\left(U_{0}, U_{1}\right)$ be a pair of subsets $U_{0}, U_{1} \subseteq V$ satisfying the following conditions:

(i) $\left|U_{0}\right|,\left|U_{1}\right| \geq \delta_{0}(\vec{G})$;

(ii) $\left|U_{0} \cap U_{1}\right| \leq((1 / 3)-21 \varepsilon) n$;

(iii) $\vec{G}$ admits no directed paths $u_{0} \rightarrow v \rightarrow u_{1}$, where $u_{0} \in U_{0}$ and $u_{1} \in U_{1}$.

Then, there exist independent sets $I_{0} \subseteq U_{0} \backslash U_{1}$ and $I_{1} \subseteq U_{1} \backslash U_{0}$ with sizes

$$
\left|I_{0}\right| \geq\left|U_{0} \backslash U_{1}\right|-7 \varepsilon n \geq 20 \varepsilon n \quad \text { and } \quad\left|I_{1}\right| \geq\left|U_{1} \backslash U_{0}\right|-7 \varepsilon n \geq 20 \varepsilon n \text {. }
$$

Remark 4.3. In many applications of Fact 4.2 , the pair $\left(U_{0}, U_{1}\right)$ will satisfy $U_{0} \cap U_{1}=\emptyset$.

Fact 4.4. Fix an integer $\ell \geq 4$ and an $\varepsilon \in(0,1 /(54))$. Let $\vec{G}=(V, \vec{E})$ be an oriented graph on $n \geq$ $n_{0}(\ell, \varepsilon)$ many vertices which admits no closed directed $\ell$-walk, but which satisfies $\delta_{0}(\vec{G}) \geq((1 / 3)-\varepsilon) n$. Let $(x, y, z, x)$ be a directed 3-cycle $\vec{C}_{3}$ in $\vec{G}$, and assume that neither $x$ nor $y$ belongs to a directed 4 -cycle $\vec{C}_{4}$. Then, $\left|N_{\vec{G}}^{-}(x) \cap N_{\vec{G}}^{+}(y)\right| \geq((1 / 3)-18 \varepsilon) n$.

We now prove Proposition 3.2 and distinguish whether or not $\ell=5$.

4.1. Proof of Proposition 3.2 when $\ell \neq 5$. Fix $\lambda_{0}>0$. Define the promised constant

$$
\beta=\beta\left(\lambda_{0}\right)=\min \left\{\frac{1}{21} \lambda_{0}, \frac{1}{3}-0.3025, \frac{1}{55}\right\} .
$$

Fix an integer $\ell \geq 4$, where $\ell \neq 5$. Let $\vec{H}=(V, \vec{E})$ be an $m$-vertex oriented graph, where $m \geq m_{0}\left(\lambda_{0}, \beta, \ell\right)$ is assumed to be sufficiently large whenever needed. Assume that $\vec{H}$ admits no closed directed $\ell$-walk but satisfies $\delta_{0}(\vec{H}) \geq((1 / 3)-\beta) m$. We prove that $\vec{H}$ is $\lambda_{0}$-extremal.

The central observation of the proof is that $\vec{H}$ admits directed triangles, since otherwise with

$$
\delta_{0}(\vec{H}) \geq\left(\frac{1}{3}-\beta\right) m \stackrel{310}{\geq}(0.3025) m
$$

Fact 4.1 would guarantee a directed $\ell$-cycle $\vec{C}_{\ell}$ in $\vec{H}$, contradicting our hypothesis. Thus, fix a directed 3-cycle $\left(v_{0}, v_{1}, v_{2}, v_{0}\right)$ in $\vec{H}$. Our observation in (27) guarantees that no vertex $v_{i} \in\left\{v_{0}, v_{1}, v_{2}\right\}$ can belong to a directed 4 -cycle $\vec{C}_{4}$ lest $\vec{H}$ admits a closed directed $\ell$-walk. For fixed $i \in \mathbb{Z}_{3}$, we define

$$
U_{i}=N_{\vec{H}}^{-}\left(v_{i}\right) \cap N_{\vec{H}}^{+}\left(v_{i+1}\right) .
$$

Then $U_{0}, U_{1}$, and $U_{2}$ are pairwise disjoint because $\vec{H}$ is an oriented graph. By our choice of $\beta<1 /(54)$ in (31), and by no $v_{j} \in\left\{v_{0}, v_{1}, v_{2}\right\}$ belonging to a directed 4 -cycle $\vec{C}_{4}$, Fact 4.4 guarantees that

$$
\left|U_{i}\right|=\left|N_{\vec{H}}^{-}\left(v_{i}\right) \cap N_{\vec{H}}^{+}\left(v_{i+1}\right)\right| \geq\left(\frac{1}{3}-18 \beta\right) n .
$$

We claim that each $u_{i} \in U_{i}$ satisfies

$$
\left|N_{\vec{H}}^{+}\left(u_{i}\right) \cap U_{i+1}\right| \geq\left(\frac{1}{3}-45 \beta\right) n .
$$


If true, any partition $V=V_{0} \cup V_{1} \cup V_{2}$, where $U_{j} \subseteq V_{j}$ for each $j \in \mathbb{Z}_{3}$, is $\lambda_{0}$-extremal since

$$
\begin{aligned}
e_{\vec{H}}\left(V_{i}, V_{i+1}\right) \geq e_{\vec{H}}\left(U_{i}, U_{i+1}\right)= & \sum_{u_{i} \in U_{i}} \mid \\
& N_{\vec{H}}^{+}\left(u_{i}\right) \cap U_{i+1}|\stackrel{\sqrt[34]{3}}{\geq}| U_{i} \mid\left(\frac{1}{3}-45 \beta\right) n \\
& \stackrel{\sqrt{33}}{\geq}\left(\frac{1}{3}-45 \beta\right)\left(\frac{1}{3}-18 \beta\right) n^{2} \geq\left(\frac{1}{9}-21 \beta\right) n^{2} \stackrel{31}{\geq}\left(\frac{1}{9}-\lambda_{0}\right) n^{2} .
\end{aligned}
$$

To prove (34), fix $i \in \mathbb{Z}_{3}$, and w.l.o.g. assume $i=0$. Fix $u_{0} \in U_{0}=N_{\vec{H}}^{-}\left(v_{0}\right) \cap N_{\vec{H}}^{+}\left(v_{1}\right)$. Then $\left(v_{1}, u_{0}, v_{0}, v_{1}\right)$ is a directed 3 -cycle $\vec{C}_{3}$, and so (27) gives that $u_{0}$ can belong to no directed 4-cycle $\vec{C}_{4}$. As such, Fact 4.4 (applied to $\left.\left(v_{1}, u_{0}, v_{0}, v_{1}\right)\right)$ guarantees that

$$
\left|N_{\vec{H}}^{-}\left(v_{1}\right) \cap N_{\vec{H}}^{+}\left(u_{0}\right)\right| \geq\left(\frac{1}{3}-18 \beta\right) n,
$$

which isn't yet (34), but it will be very close. With an error we can control, we shall 'replace' $N_{\vec{H}}^{-}\left(v_{1}\right)$ in (35) with $U_{1} \subseteq N_{\vec{H}}^{-}\left(v_{1}\right)$ from (32). We claim this error will be small if

$$
\operatorname{deg}_{\vec{H}}^{-}\left(v_{1}\right) \leq\left(\frac{1}{3}+9 \beta\right) n
$$

Indeed, if (36) holds, then we would have

$$
\left|N_{\vec{H}}^{-}\left(v_{1}\right) \backslash U_{1}\right| \stackrel{\sqrt[32]{=}}{=} \operatorname{deg}_{\vec{H}}^{-}\left(v_{1}\right)-\left|U_{1}\right| \stackrel{\sqrt[33]{\leq}}{\leq} \operatorname{deg}_{\vec{H}}^{-}\left(v_{1}\right)-\left(\frac{1}{3}-18 \beta\right) n \stackrel{\sqrt[36]{\leq}}{\leq} 27 \beta n,
$$

and so comparing (35) with (37) yields

$$
\left|N_{\vec{H}}^{+}\left(u_{0}\right) \cap U_{1}\right|+27 \beta n \stackrel{37}{\geq}\left|N_{\vec{H}}^{+}\left(u_{0}\right) \cap N_{\vec{H}}^{-}\left(v_{1}\right)\right| \stackrel{35}{\geq}\left(\frac{1}{3}-18 \beta\right) n,
$$

which gives (34). It thus remains to prove that (36) holds.

To prove (36), we will apply Fact 4.2 to the pair $\left(N_{\vec{H}}^{-}\left(v_{1}\right), N_{\vec{H}}^{+}\left(v_{1}\right)\right)$. Note that the hypotheses (i)-(iii) of Fact 4.2 are met by $\left(N_{\vec{H}}^{-}\left(v_{1}\right), N_{\vec{H}}^{+}\left(v_{1}\right)\right)$ since $\left|N_{\vec{H}}^{-}\left(v_{1}\right)\right|,\left|N_{\vec{H}}^{+}\left(v_{1}\right)\right| \geq \delta_{0}(\vec{H})$, since $\left|N_{\vec{H}}^{-}\left(v_{1}\right) \cap N_{\vec{H}}^{+}\left(v_{1}\right)\right|=0$, and since there are no paths $u^{+} \rightarrow v \rightarrow u^{-}$with $u^{+} \in N_{\vec{H}}^{+}\left(v_{1}\right)$ and $u^{-} \in N_{\vec{H}}^{-}\left(v_{1}\right)$ lest $\left(v_{1}, u^{+}, v, u^{-}, v_{1}\right)$ is a directed 4-cycle containing $v_{1}$. Fact 4.2 guarantees an independent set $I_{v_{1}} \subseteq N_{\vec{H}}^{-}\left(v_{1}\right)$ of size

$$
\left|I_{v_{1}}\right| \geq \operatorname{deg}_{\vec{H}}^{-}\left(v_{1}\right)-7 \beta n \geq \delta_{0}(\vec{H})-7 \beta n \geq\left(\frac{1}{3}-\beta\right) n-7 \beta n \geq\left(\frac{1}{3}-8 \beta\right) n \stackrel{31}{>} 0,
$$

so fix $w_{1} \in I_{v_{1}}$. Now, $N_{\vec{H}}^{+}\left(w_{1}\right) \cup N_{\vec{H}}^{-}\left(w_{1}\right) \cup I_{v_{1}} \subseteq V$ is a pairwise disjoint union, in which case

$$
\begin{aligned}
n \geq \operatorname{deg}_{\vec{H}}^{+}\left(w_{1}\right)+\operatorname{deg}_{\vec{H}}^{-}\left(w_{1}\right)+\left|I_{v_{1}}\right| \stackrel{\text { 38 }}{\geq} \operatorname{deg}_{\vec{H}}^{+}\left(w_{1}\right)+\operatorname{deg}_{\vec{H}}^{-}\left(w_{1}\right)+\operatorname{deg}_{\vec{H}}^{-}\left(v_{1}\right)-7 \beta n \\
\quad \geq 2 \delta_{0}(\vec{H})+\operatorname{deg}_{\vec{H}}^{-}\left(v_{1}\right)-7 \beta n \geq 2\left(\frac{1}{3}-\beta\right) n+\operatorname{deg}_{\vec{H}}^{-}\left(v_{1}\right)-7 \beta n=\operatorname{deg}_{\vec{H}}^{-}\left(v_{1}\right)+\left(\frac{2}{3}-9 \beta\right) n,
\end{aligned}
$$

from which (36) now follows.

4.2. Proof of Proposition 3.2 when $\ell=5$. To prove Proposition 3.2 when $\ell=5$, we use Facts 4.14 .4 together with the following two additional facts (which are also proven in Section 5).

Fact 4.5. Fix an integer $\ell \geq 4$ and an $\varepsilon>0$. Let $\vec{G}=(V, \vec{E})$ be an oriented graph on $n \geq n_{0}(\ell, \varepsilon)$ many vertices which admits no closed directed $\ell$-walk. Then, $\delta^{+}(\vec{G}) \leq((1 / 3)+\varepsilon) n$ and $\delta^{-}(\vec{G}) \leq((1 / 3)+\varepsilon) n$.

Fact 4.6. For all $\lambda>0$, there exists $\varepsilon=\varepsilon(\lambda)>0$ so that every oriented graph $\vec{G}=(V, \vec{E})$ on $n \geq n_{0}(\lambda, \varepsilon)$ many vertices with $\delta_{0}(\vec{G}) \geq((1 / 3)-\varepsilon) n$ will be $\lambda$-extremal, provided $\vec{G}$ has:

(1) a partition $V=V_{0} \cup V_{1} \cup V_{2}$ with $\left|V_{1}\right|,\left|V_{2}\right| \geq((1 / 3)-\varepsilon) n$ and $e_{\vec{G}}\left(V_{1}\right), e_{\vec{G}}\left(V_{2}\right), e_{\vec{G}}\left(V_{2}, V_{1}\right) \leq \varepsilon n^{2}$,

(2) or no transitive triangles. 
Now, let $\lambda_{0}>0$ be given. Let

$$
\varepsilon_{\mathrm{Fct}[4.6]}=\varepsilon_{\mathrm{Fct}[4.6}\left(\lambda=\lambda_{0}\right)>0
$$

be the constant guaranteed by Fact 4.6 . We define the promised constant

$$
\beta=\frac{1}{109} \varepsilon_{\mathrm{Fct} 4.6 .}
$$

Let $\vec{H}=(V, \vec{E})$ be an $m$-vertex oriented graph, where in all that follows we assume $m \geq m_{0}\left(\lambda_{0}, \varepsilon_{\mathrm{Fct}} \overleftarrow{4.6 \text {, }}, \beta\right)$ is sufficiently large. Assume that $\vec{H}$ admits no closed directed 5 -walks, i.e., directed 5 -cycles $\vec{C}_{5}$, but which satisfies $\delta_{0}(\vec{H}) \geq((1 / 3)-\beta) m$. We prove that $\vec{H}$ is $\lambda_{0}$-extremal.

For sake of argument, we assume that $\vec{H}$ admits some transitive triangles, as otherwise by our choice of $\beta$ and $\varepsilon_{\mathrm{Fct}(4.6]}$ in (39) and (40), Conclusion (2) of Fact 4.6 would give that $\vec{H}$ is $\lambda_{0}$-extremal. For the remainder of the proof, we fix a transitive triangle $(x, y),(x, z),(y, z) \in \vec{E}$. Let $I=I_{x, z}=N_{\vec{H}}^{-}(x) \cap N_{\vec{H}}^{+}(z)$, which is an independent set lest $(a, b) \in \vec{E} \cap(I \times I)$ gives the directed 5-cycle $(x, y, z, a, b, x)$. Our first main observation is that $I$ is 'large'.

\section{Claim 4.7.}

$$
|I| \geq\left(\frac{1}{3}-21 \beta\right) n
$$

Proof of Claim 4.7. Assume for contradiction that (41) fails to hold. We will apply Fact 4.2 to the pair $\left(N_{\vec{H}}^{-}(x), N_{\vec{H}}^{+}(z)\right)$. Note that the hypotheses (i)-(iii) are met by $\left(N_{\vec{H}}^{-}(x), N_{\vec{H}}^{+}(z)\right)$ since $\left.\left|N_{\vec{H}}^{-}(x)\right|, \mid N_{\vec{H}}^{+}(z)\right) \mid \geq$ $\delta_{0}(\vec{H})$, since $\left.\mid N_{\vec{H}}^{-}(x) \cap N_{\vec{H}}^{+}(z)\right) \mid \leq((1 / 3)-21 \beta) n$ on account that (41) failed, and since there are no paths $u^{+} \rightarrow v \rightarrow u^{-}$with $u^{+} \in N_{\vec{H}}^{+}(z)$ and $u^{-} \in N_{\vec{H}}^{-}(x)$ lest $\left(x, z, u^{+}, v, u^{-}, x\right)$ is a directed 5-cycle $\vec{C}_{5}$. Fact 4.2 guarantees disjoint independent sets $I_{x} \subseteq N_{\vec{H}}^{-}(x) \backslash N_{\vec{H}}^{+}(z)$ and $I_{z} \subseteq N_{\vec{H}}^{+}(z) \backslash N_{\vec{H}}^{-}(x)$ (disjoint also from $I$ ) with sizes

$$
\begin{aligned}
\left|I_{x}\right| \geq\left|N_{\vec{H}}^{-}(x) \backslash N_{\vec{H}}^{+}(z)\right|-7 \beta n=\operatorname{deg}_{\vec{H}}^{-}(x)-|I|-7 \beta n \geq 20 \beta n \\
\quad \text { and }\left|I_{z}\right| \geq\left|N_{\vec{H}}^{+}(z) \backslash N_{\vec{H}}^{-}(x)\right|-7 \beta n=\operatorname{deg}_{\vec{H}}^{+}(z)-|I|-7 \beta n \geq 20 \beta n .
\end{aligned}
$$

Fix $a_{x} \in I_{x}$ and $b_{z} \in I_{z}$. One may check that

$$
N_{\vec{H}}^{-}\left(a_{x}\right) \cap N_{\vec{H}}^{+}\left(b_{z}\right)=N_{\vec{H}}^{-}\left(a_{x}\right) \cap I=N_{\vec{H}}^{+}\left(b_{z}\right) \cap I=N_{\vec{H}}^{-}\left(a_{x}\right) \cap I_{z}=N_{\vec{H}}^{+}\left(b_{z}\right) \cap I_{x}=\emptyset .
$$

Thus, together with the independence of $I, I_{x}$, and $I_{z}$, we have that $I \cup I_{x} \cup I_{y} \cup N^{-}\left(a_{x}\right) \cup N^{+}\left(b_{z}\right) \subseteq V$ is a pairwise disjoint union, and so

$$
\begin{array}{r}
n \geq|I|+\left|I_{x}\right|+\left|I_{z}\right|+\operatorname{deg}_{\vec{H}}^{-}\left(a_{x}\right)+\operatorname{deg}_{\vec{H}}^{+}\left(b_{z}\right) \stackrel{\sqrt[42]{\geq}}{\geq} \operatorname{deg}_{\vec{H}}^{-}(x)+\operatorname{deg}_{\vec{H}}^{+}(z)-|I|+\operatorname{deg}_{\vec{H}}^{-}\left(a_{x}\right)+\operatorname{deg}_{\vec{H}}^{+}\left(b_{z}\right)-14 \beta n \\
\geq 4 \delta_{0}(\vec{H})-|I|-14 \beta n \stackrel{\text { hyp }}{\geq} 4\left(\frac{1}{3}-\beta\right) n-|I|-14 \beta n=n-|I|+\left(\frac{1}{3}-18 \beta\right) n,
\end{array}
$$

from which $|I| \geq((1 / 3)-18 \beta) n$ follows, and contradicts our assumption that (41) failed to hold.

Continuing the proof of Proposition 3.2, we attempt to meet Condition (1) of Fact 4.6 to $\vec{H}$ with $V_{1}=I$ and with $V_{2}$ which we now define. For the remainder of the proof, fix $v \in I$ and take $V_{2}=I_{v}$ to be a maximal independent set in $N_{\vec{H}}^{+}(v)$ or $N_{\vec{H}}^{-}(v)$. In the former case, $0=e_{\vec{H}}\left(V_{1}\right)=e_{\vec{H}}\left(V_{2}\right)=e_{\vec{H}}\left(V_{2}, V_{1}\right)$ since each of $V_{1}=I$ and $V_{2}=I_{v}$ is independent and since

$$
a \in N_{\vec{H}}^{+}(v) \text { forbids } b \in N_{\vec{H}}^{+}(a) \cap I,
$$

lest $(x, z, v, a, b, x)$ is a directed 5-cycle $\vec{C}_{5}$ in $\vec{H}$. In the latter case, $0=e_{\vec{H}}\left(V_{1}\right)=e_{\vec{H}}\left(V_{2}\right)=e_{\vec{H}}\left(V_{1}, V_{2}\right)$, where the last equality holds by $a \in N_{\vec{G}}^{-}(v)$ forbidding $b \in N_{\vec{H}}^{-}(a) \cap I$ lest $(x, z, b, a, v, x)$ is a directed 5-cycle $\vec{C}_{5}$. In either case, we make the following claim.

\section{Claim 4.8.}

$$
\left|V_{2}\right|=\left|I_{v}\right| \geq\left(\frac{1}{3}-24 \beta\right) n
$$


If Claim 4.8 holds, then together with (41) and the considerations above, the partition $V=V_{0} \cup V_{1} \cup V_{2}$, where $V_{0}=V \backslash\left(V_{1} \cup V_{2}\right)$, meets the hypotheses of Fact 4.6. By our choice of $\varepsilon_{\mathrm{Fct} \text { 4.6 }}$ and $\beta$ in (39) and (40), Fact 4.6 guarantees that $V=V_{0} \cup V_{1} \cup V_{2}$ is a $\lambda_{0}$-extremal partition of $\vec{H}$. Thus, the proof of Proposition 3.2 when $\ell=5$ will be complete upon proving Claim 4.8 .

Proof of Claim 4.8. Assume for contradiction that the $\vec{H}$-subgraphs $\vec{H}\left[N_{\vec{H}}^{+}(v)\right]$ and $\vec{H}\left[N_{\vec{H}}^{-}(v)\right]$ induced respectively on $N_{\vec{H}}^{+}(v)$ and $N_{\vec{H}}^{-}(v)$ satisfy

$$
\alpha\left(\vec{H}\left[N_{\vec{H}}^{+}(v)\right]\right)<\left(\frac{1}{3}-24 \beta\right) n \quad \text { and } \quad \alpha\left(\vec{H}\left[N_{\vec{H}}^{-}(v)\right]\right)<\left(\frac{1}{3}-24 \beta\right) n,
$$

where $\alpha(\cdot)$ denotes the independence number. Since

$$
\left|N_{\vec{H}}^{+}(v)\right|=\operatorname{deg}_{\vec{H}}^{+}(v) \geq \delta_{0}(\vec{H}) \geq\left(\frac{1}{3}-\beta\right) n>\left(\frac{1}{3}-24 \beta\right) n>\alpha\left(\vec{H}\left[N_{\vec{H}}^{+}(v)\right]\right),
$$

$\vec{H}\left[N_{\vec{H}}^{+}(v)\right]$ admits edges $(a, b) \in \vec{E}$. We fix one such and will observe that

$$
N_{\vec{H}}^{+}(b) \cap N_{\vec{H}}^{+}(v) \neq \emptyset
$$

Indeed, assuming otherwise the set $N_{\vec{H}}^{+}(b) \cap N_{\vec{H}}^{-}(v)$ satisfies

$$
\left|N_{\vec{H}}^{+}(b) \cap N_{\vec{H}}^{-}(v)\right|=\left|N_{\vec{H}}^{+}(b)\right|+\left|N_{\vec{H}}^{-}(v)\right|-\left|N_{\vec{H}}^{+}(b) \cup N_{\vec{H}}^{-}(v)\right| .
$$

From $\emptyset=N_{\vec{H}}^{+}(b) \cap N_{\vec{H}}^{+}(v)=N_{\vec{H}}^{+}(b) \cap I$ (cf. (43) and (46)) we infer $N_{\vec{H}}^{+}(b) \subseteq V \backslash\left(N_{\vec{H}}^{+}(v) \cup I\right)$, and from $\emptyset=N_{\vec{H}}^{-}(v) \cap N_{\vec{H}}^{+}(v)=N_{\vec{H}}^{-}(v) \cap I$ (recall that $I$ is independent) we infer $N_{\vec{H}}^{+}(b) \cup N_{\vec{H}}^{-}(v) \subseteq V \backslash\left(N_{\vec{H}}^{+}(v) \cup I\right)$, where $N_{\vec{H}}^{+}(v) \cup I$ is a disjoint union by the independence of $I$. Thus,

$$
\begin{aligned}
\left|N_{\vec{H}}^{+}(b) \cap N_{\vec{H}}^{-}(v)\right| & \stackrel{47}{\geq} \operatorname{deg}_{\vec{H}}^{+}(b)+\operatorname{deg}_{\vec{H}}^{-}(v)-\left(n-\operatorname{deg}_{\vec{H}}^{+}(v)-|I|\right) \\
& \geq 3 \delta_{0}(\vec{H})-n+|I| \geq 3\left(\frac{1}{3}-\beta\right) n-n+|I| \stackrel{41}{\geq}\left(\frac{1}{3}-24 \beta\right) n \stackrel{45}{>} \alpha\left(\vec{H}\left[N_{\vec{H}}^{-}(v)\right]\right) .
\end{aligned}
$$

Consequently, there exists an edge $(c, d) \in \vec{E}$ with $c, d \in N_{\vec{H}}^{+}(b) \cap N_{\vec{H}}^{-}(v)$, in which case $(v, a, b, c, d, v)$ is a directed 5 -cycle $\vec{C}_{5}$ in $\vec{H}$. This proves (46).

Now, define

$$
C=\left\{c \in N_{\vec{H}}^{+}(v): \exists \text { a directed path on } 3 \text { vertices contained in } \vec{H}\left[N_{\vec{H}}^{+}(v)\right] \text { that ends in } c\right\} .
$$

Note that (46) implies that $C$ is non-empty. By this definition, every element $c \in C$ satisfies

$$
N_{\vec{H}}^{+}(c) \cap N_{\vec{H}}^{+}(v)=N_{\vec{H}}^{+}(c) \cap C .
$$

Since the $\vec{H}$-subgraph $\vec{H}[C]$ induced on $C$ admits no directed 5-cycles $\vec{C}_{5}$, Fact 4.5 guarantees the existence of a vertex $c_{0} \in C \subseteq N_{\vec{H}}^{+}(v)$ (cf. (48) ) so that

$$
\left|N_{\vec{H}}^{+}\left(c_{0}\right) \cap N_{\vec{H}}^{+}(v)\right| \stackrel{499}{=}\left|N_{\vec{H}}^{+}\left(c_{0}\right) \cap C\right| \leq\left\{\begin{array}{cc}
\left(\frac{1}{3}+\beta\right)|C| & \text { if }|C|=\Omega(1) \\
|C| & \text { else }
\end{array}\right\} \leq\left(\frac{1}{3}+\beta\right)\left|N_{\vec{H}}^{+}(v)\right| .
$$

Consider now $N_{\vec{H}}^{+}\left(c_{0}\right) \cap N_{\vec{H}}^{-}(v)=\left(N_{\vec{H}}^{+}\left(c_{0}\right) \backslash C\right) \cap N_{\vec{H}}^{-}(v)$, where $C \subseteq N_{\vec{H}}^{+}(v)$ from (48) but where $N_{\vec{H}}^{+}(v) \cap N_{\vec{H}}^{-}\left(v_{i}\right)=\emptyset$. Then

$$
\begin{array}{r}
\left|N_{\vec{H}}^{+}\left(c_{0}\right) \cap N_{\vec{H}}^{-}(v)\right|=\left|\left(N_{\vec{H}}^{+}\left(c_{0}\right) \backslash C\right) \cap N_{\vec{H}}^{-}(v)\right|=\left|\left(N_{\vec{H}}^{+}\left(c_{0}\right) \backslash C\right)\right|+\left|N_{\vec{H}}^{-}(v)\right|-\left|\left(N_{\vec{H}}^{+}\left(c_{0}\right) \backslash C\right) \cup N_{\vec{H}}^{-}(v)\right| \\
\stackrel{500}{\geq} \operatorname{deg}_{\vec{H}}^{+}\left(c_{0}\right)-\left(\frac{1}{3}+\beta\right) \operatorname{deg}_{\vec{H}}^{+}(v)+\operatorname{deg}_{\vec{H}}^{-}(v)-\left|\left(N_{\vec{H}}^{+}\left(c_{0}\right) \backslash C\right) \cup N_{\vec{H}}^{-}(v)\right| \\
\stackrel{49}{=} \operatorname{deg}_{\vec{H}}^{+}\left(c_{0}\right)-\left(\frac{1}{3}+\beta\right) \operatorname{deg}_{\vec{H}}^{+}(v)+\operatorname{deg}_{\vec{H}}^{-}(v)-\left|\left(N_{\vec{H}}^{+}\left(c_{0}\right) \backslash N_{\vec{H}}^{+}(v)\right) \cup N_{\vec{H}}^{-}(v)\right| .
\end{array}
$$


Using (43) and the independence of $I$, the last union resides in $V \backslash\left(N_{\vec{H}}^{+}(v) \cup I\right)$, and so

$$
\begin{aligned}
& \mid N_{\vec{H}}^{+}\left(c_{0}\right) \cap N_{\vec{H}}^{-}(v) \mid \geq \operatorname{deg}_{\vec{H}}^{+}\left(c_{0}\right)-\left(\frac{1}{3}+\beta\right) \operatorname{deg}_{\vec{H}}^{+}(v)+\operatorname{deg}_{\vec{H}}^{-}(v)-\left(n-\operatorname{deg}_{\vec{H}}^{+}(v)-|I|\right) \\
&= \operatorname{deg}_{\vec{H}}^{+}\left(c_{0}\right)+\operatorname{deg}_{\vec{H}}^{-}(v)+\left(\frac{2}{3}-\beta\right) \operatorname{deg}_{\vec{H}}^{+}(v)+|I|-n \geq\left(\frac{8}{3}-\beta\right) \delta_{0}(\vec{H})+|I|-n \\
& \quad \geq\left(\frac{8}{3}-\beta\right)\left(\frac{1}{3}-\beta\right) n+|I|-n \stackrel{441}{\geq}\left(\frac{8}{3}-\beta\right)\left(\frac{1}{3}-\beta\right) n+\left(\frac{1}{3}-21 \beta\right) n-n \geq\left(\frac{2}{9}-24 \beta\right) n,
\end{aligned}
$$

which is positive by (40). Now, (48) and (51) render a directed path $a \rightarrow b \rightarrow c_{0} \rightarrow d$ where $a, b, c_{0} \in$ $N_{\vec{H}}^{+}(v)$ and $d \in N_{\vec{H}}^{+}\left(c_{0}\right) \cap N_{\vec{H}}^{-}(v)$, in which case $\left(v, a, b, c_{0}, d, v\right)$ is a directed 5-cycle in $\vec{H}$. Thus, our assumption in (45) is incorrect, which completes the proof of Claim 4.8 .

\section{Proofs of Facts 4.24 .6}

The easiest proof here is that of Fact 4.5, which we give immediately. Fix an integer $\ell \geq 4$ and fix $\varepsilon>0$. Let $\vec{G}=(V, \vec{E})$ be an oriented graph on $n \geq n_{0}(\ell, \varepsilon)$ many vertices which admits no closed directed $\ell$-walk. The latter conclusion of Fact 4.5 follows from the former by reversing the orientations on $\vec{E}$. If the former fails, then Proposition 3.1 ensures a large $m$-vertex subgraph $\vec{H} \subseteq \vec{G}$ satisfying

$$
\delta_{0}(\vec{H}) \geq\left(\frac{\delta^{+}(\vec{G})}{n}-\frac{\varepsilon}{2}\right) m \geq\left(\frac{1}{3}+\frac{\varepsilon}{2}\right) m \geq \frac{m+1}{3},
$$

and so Theorem 1.5 guarantees a directed $\ell$-cycle $\vec{C}_{\ell}$ in $\vec{H}$, and hence in $\vec{G}$.

Proof of Fact 4.2. Fix an integer $\ell \geq 4$ and an $\varepsilon \in(0,1 /(11)]$. Let $\vec{G}=(V, \vec{E})$ be an oriented graph on $n \geq n_{0}(\ell, \varepsilon)$ many vertices which admits no closed directed $\ell$-walk, but which satisfies $\delta_{0}(\vec{G}) \geq$ $((1 / 3)-\varepsilon) n$. Let $\left(U_{0}, U_{1}\right)$ be a pair of subsets satisfying (i)-(iii) in the hypotheses of Fact 4.2 . We prove that there exist independent sets $I_{0} \subseteq U_{0} \backslash U_{1}$ and $I_{1} \subseteq U_{1} \backslash U_{0}$ satisfying (30). To that end, define

$$
T_{0}=\left\{u_{0} \in U_{0} \backslash U_{1}: N_{\vec{G}}^{-}\left(u_{0}\right) \cap\left(U_{0} \backslash U_{1}\right) \neq \emptyset\right\} \quad \text { and } \quad T_{1}=\left\{u_{1} \in U_{1} \backslash U_{0}: N_{\vec{G}}^{+}\left(u_{1}\right) \cap\left(U_{1} \backslash U_{0}\right) \neq \emptyset\right\} .
$$

For fixed $j \in \mathbb{Z}_{2}$, the set $I_{j}=U_{j} \backslash\left(U_{j+1} \cup T_{j}\right)$ is independent and of size $\left|I_{j}\right|=\left|U_{j} \backslash U_{j+1}\right|-\left|T_{j}\right|$, so to prove (30) we will prove $\left|T_{j}\right| \leq 7 \varepsilon n$. In particular, our argument will show that $\left|T_{0}\right| \geq \varepsilon n$ and $\left|T_{1}\right| \geq \varepsilon n$ can't both hold, and that $\left|T_{j}\right|<\varepsilon n$ implies $\left|T_{j+1}\right| \leq 7 \varepsilon n$. It remains to verify these details.

Write $U=U_{0} \cup U_{1}$, and define

$$
S_{0}=N^{+}\left(T_{0}\right) \backslash U \quad \text { and } \quad S_{1}=N^{-}\left(T_{1}\right) \backslash U, \quad \text { where } \quad S_{0} \cap S_{1} \stackrel{(\text { iii) }}{=} \emptyset .
$$

For $j \in \mathbb{Z}_{2}$, we will verify the implications

$$
\left|T_{j}\right| \geq \varepsilon n \quad \Longrightarrow \quad\left|S_{j}\right| \geq \delta_{0}(\vec{G})-\left(\frac{1}{3}+\varepsilon\right)\left|T_{j}\right| \quad \Longrightarrow \quad\left|T_{j+1}\right|<\varepsilon n .
$$

Indeed, $\vec{G}\left[T_{j}\right]$ is a large oriented graph with no closed directed $\ell$-walks, so Fact 4.5 guarantees $t_{j} \in T_{j}$ :

$$
\left|N_{\vec{G}}^{+}\left(t_{0}\right) \cap T_{0}\right| \leq\left(\frac{1}{3}+\varepsilon\right)\left|T_{0}\right| \quad \text { and } \quad\left|N_{\vec{G}}^{-}\left(t_{1}\right) \cap T_{1}\right| \leq\left(\frac{1}{3}+\varepsilon\right)\left|T_{1}\right| .
$$

By definition, there exist $u_{0} \in U_{0} \backslash U_{1}$ and $u_{1} \in U_{1} \backslash U_{0}$ with $\left(u_{0}, t_{0}\right),\left(t_{1}, u_{1}\right) \in \vec{E}$, where $N^{+}\left(t_{0}\right) \cap T_{0}=$ $N^{+}\left(t_{0}\right) \cap\left(U_{0} \backslash U_{1}\right)$ and $N^{-}\left(t_{1}\right) \cap T_{1}=N^{-}\left(t_{1}\right) \cap\left(U_{1} \backslash U_{0}\right)$ hold. Moreover, $u \in N_{\vec{G}}^{+}\left(t_{0}\right) \cap U_{1}$ is impossible lest $\left(u_{0}, t_{0}, u\right)$ violates (iii), and $N_{\vec{G}}^{-}\left(t_{1}\right) \cap U_{0} \neq \emptyset$ is similarly impossible. Altogether,

$$
\begin{aligned}
\operatorname{deg}_{\vec{G}}^{+}\left(t_{0}\right)= & \left|N_{\vec{G}}^{+}\left(t_{0}\right) \cap U\right|+\left|N_{\vec{G}}^{+}\left(t_{0}\right) \backslash U\right|=\left|N_{\vec{G}}^{+}\left(t_{0}\right) \cap\left(U_{0} \backslash U_{1}\right)\right|+\left|N_{\vec{G}}^{+}\left(t_{0}\right) \backslash U\right| \\
& =\left|N_{\vec{G}}^{+}\left(t_{0}\right) \cap T_{0}\right|+\left|N_{\vec{G}}^{+}\left(t_{0}\right) \backslash U\right| \stackrel{[54]}{\leq}\left(\frac{1}{3}+\varepsilon\right)\left|T_{0}\right|+\left|N_{\vec{G}}^{+}\left(t_{0}\right) \backslash U\right| \stackrel{[52]}{\leq}\left(\frac{1}{3}+\varepsilon\right)\left|T_{0}\right|+\left|S_{0}\right|,
\end{aligned}
$$

and so the former implication of (53) holds with $j=0$. Similarly,

$$
\operatorname{deg}_{\vec{G}}^{-}\left(t_{1}\right)=\left|N_{\vec{G}}^{-}\left(t_{1}\right) \cap U\right|+\left|N_{\vec{G}}^{-}\left(t_{1}\right) \backslash U\right| \leq\left|N_{\vec{G}}^{-}\left(t_{1}\right) \cap T_{1}\right|+\left|S_{1}\right| \leq\left(\frac{1}{3}+\varepsilon\right)\left|T_{1}\right|+\left|S_{1}\right|,
$$


and so the former implication of (53) holds with $j=1$. Finally, if both $\left|T_{0}\right|,\left|T_{1}\right| \geq \varepsilon n$, then

$$
\begin{aligned}
& n \stackrel{[52]}{\geq}|U|+\left|S_{0}\right|+\left|S_{1}\right| \stackrel{\sqrt[53]{\geq}}{\geq}|U|+2 \delta_{0}(\vec{G})-\left(\frac{1}{3}+\varepsilon\right)\left(\left|T_{0}\right|+\left|T_{1}\right|\right) \\
& \geq|U|+2 \delta_{0}(\vec{G})-\left(\frac{1}{3}+\varepsilon\right)\left|U_{0} \triangle U_{1}\right|=\left(\frac{2}{3}-\varepsilon\right)\left(\left|U_{0}\right|+\left|U_{1}\right|\right)+2 \delta_{0}(\vec{G})-\left(\frac{1}{3}-2 \varepsilon\right)\left|U_{0} \cap U_{1}\right| \\
& \stackrel{(\text { i })}{\geq} 2 \delta_{0}(\vec{G})\left(\frac{5}{3}-\varepsilon\right)-\left(\frac{1}{3}-2 \varepsilon\right)\left|U_{0} \cap U_{1}\right| \stackrel{(i i)}{\geq} 2 \delta_{0}(\vec{G})\left(\frac{5}{3}-\varepsilon\right)-\left(\frac{1}{3}-2 \varepsilon\right)\left(\frac{1}{3}-21 \varepsilon\right) n \\
& \quad \geq 2 \delta_{0}(\vec{G})\left(\frac{5}{3}-\varepsilon\right)-\left(\frac{1}{9}-\frac{23}{3} \varepsilon+42 \varepsilon^{2}\right) n \geq 2\left(\frac{1}{3}-\varepsilon\right)\left(\frac{5}{3}-\varepsilon\right) n-\left(\frac{1}{9}-\frac{23}{3} \varepsilon+42 \varepsilon^{2}\right) n,
\end{aligned}
$$

from which $\varepsilon \geq 11 /(120)$ follows and contradicts the hypothesis $\varepsilon \leq 1 /(11)$. This proves (53)).

By (53), it suffices to assume for fixed $j \in \mathbb{Z}_{2}$ that $\left|T_{j}\right| \geq \varepsilon n$, and then to prove that $\left|T_{j}\right| \leq 7 \varepsilon n$. To that end, we find a vertex $z_{j+1} \in U_{j+1} \backslash T_{j+1}$ where,

(a) when $j=0$, the vertex $z_{1} \in U_{1} \backslash T_{1}$ has no in-neighbors from $U_{1} \backslash T_{1}$;

(b) when $j=1$, the vertex $z_{0} \in U_{0} \backslash T_{0}$ has no out-neighbors in $U_{0} \backslash T_{0}$.

We start by fixing $v_{j+1} \in I_{j+1}=U_{j+1} \backslash\left(U_{j} \cup T_{j+1}\right)$, which is possible by

$$
\begin{aligned}
\left|I_{j+1}\right|=\left|U_{j+1} \backslash\left(U_{j} \cup T_{j+1}\right)\right| & =\left|U_{j+1}\right|-\left|U_{0} \cap U_{1}\right|-\left|T_{j+1}\right| \stackrel{(\mathrm{i})}{\geq} \delta_{0}(\vec{G})-\left|U_{0} \cap U_{1}\right|-\left|T_{j+1}\right| \\
& \stackrel{(\mathrm{ii})}{\geq} \delta_{0}(\vec{G})-\left(\frac{1}{3}-21 \varepsilon\right) n-\left|T_{j+1}\right| \stackrel{(53)}{\geq} \delta_{0}(\vec{G})-\left(\frac{1}{3}-21 \varepsilon\right) n-\varepsilon n \geq 19 \varepsilon n .
\end{aligned}
$$

Consider $(a)$ above $(j=0)$. If $v_{1}$ has an in-neighbor $w_{1} \in U_{1} \backslash T_{1}$, then $w_{1} \in U_{0} \cap U_{1}$ because $I_{1}$ is independent. If $w_{1}$ has an in-neighbor $x_{1} \in U_{1} \backslash T_{1}$, then $x_{1} \in I_{1}$ lest we violate (iii). If $x_{1}$ has an inneighbor $y_{1} \in U_{1} \backslash T_{1}$, then $y_{1} \in U_{0} \cap U_{1}$ because $I_{1}$ is independent, but now we have violated (iii). Thus, some $z_{1} \in\left\{v_{1}, w_{1}, x_{1}, y_{1}\right\}$ has no in-neighbor within $U_{1} \backslash T_{1}$. Purely symmetric arguments establish (b).

We use $(a)$ and $(b)$ above to conclude the proof of Fact 4.2. where we first consider $j=0$. The sets $U_{1} \backslash T_{1}, S_{0}$, and $T_{0}$ are pairwise disjoint by construction, and the set $Z_{1}=N_{\vec{G}}^{-}\left(z_{1}\right)$ is disjoint from $U_{1} \backslash T_{1}$ by $(a)$ and is disjoint from each of $S_{0}$ and $T_{0}$ by (iii). When $j=1$, the sets $Z_{0}=N^{+}\left(z_{0}\right), U_{0} \backslash T_{0}, S_{1}$, and $T_{1}$ are similarly pairwise disjoint. Thus, for whichever $j \in \mathbb{Z}_{2}$ satisfies $\left|T_{j}\right| \geq \varepsilon n$, we have

$$
\begin{aligned}
n \geq & \left|Z_{j+1}\right|+\left|U_{j+1} \backslash T_{j+1}\right|+\left|S_{j}\right|+\left|T_{j}\right| \geq \delta_{0}(\vec{G})+\left|U_{j+1}\right|-\left|T_{j+1}\right|+\left|S_{j}\right|+\left|T_{j}\right| \\
& \stackrel{(53)}{>} 2 \delta_{0}(\vec{G})+\left|U_{j+1}\right|-\varepsilon n+\left(\frac{2}{3}-\varepsilon\right)\left|T_{j}\right| \stackrel{(\mathrm{i})}{\geq} 3 \delta_{0}(\vec{G})-\varepsilon n+\left(\frac{2}{3}-\varepsilon\right)\left|T_{j}\right| \geq n-4 \varepsilon n+\left(\frac{2}{3}-\varepsilon\right)\left|T_{j}\right|,
\end{aligned}
$$

from which $\left|T_{j}\right| \leq(132 /(19)) \varepsilon n<7 \varepsilon n$ follows from $\varepsilon \in(0,1 /(11)]$.

Proof of Fact 4.4. Fix an integer $\ell \geq 4$ and an $\varepsilon \in(0,1 /(54))$. Let $\vec{G}=(V, \vec{E})$ be an oriented graph on $n \geq n_{0}(\ell, \varepsilon)$ many vertices which admits no closed directed $\ell$-walk, but which satisfies $\delta_{0}(\vec{G}) \geq$ $((1 / 3)-\varepsilon) n$. Let $(x, y, z, x)$ be a directed 3 -cycle $\vec{C}_{3}$ in $\vec{G}$, and assume that neither $x$ nor $y$ belongs to a directed 4-cycle $\vec{C}_{4}$. Assume, on the contrary, that

$$
\left|N_{\vec{G}}^{-}(x) \cap N_{\vec{G}}^{+}(y)\right|<\left(\frac{1}{3}-18 \varepsilon\right) n .
$$

We will show that our assumption in (55) implies

$$
N_{\vec{G}}^{+}(x) \cap N_{\vec{G}}^{-}(y) \neq \emptyset,
$$

in which case an element $v \in N_{\vec{G}}^{+}(x) \cap N_{\vec{G}}^{-}(y)$ would result in the directed 4-cycle $(x, v, y, z, x)$ containing both $x$ and $y$. We now establish the details for (56).

First, we apply Fact 4.2 to each of the pairs $\left(X_{0}, X_{1}\right)$ and $\left(Y_{0}, Y_{1}\right)$, where

$$
X_{0}=N_{\vec{G}}^{+}(x), \quad X_{1}=N_{\vec{G}}^{-}(x), \quad Y_{0}=N_{\vec{G}}^{+}(y), \quad Y_{1}=N_{\vec{G}}^{-}(y) .
$$

Note that the hypotheses of Fact 4.2 are met since $\vec{G}$ admits no closed directed $\ell$-walks but satisfies $\delta_{0}(\vec{G}) \geq((1 / 3)-\varepsilon) n$ for $0<\varepsilon<1 /(54)<1 /(11)$, and where e.g. $\left(X_{0}, X_{1}\right)$ satisfies the hypotheses (i)(iii) of Fact 4.2 since $\left|X_{0}\right|,\left|X_{1}\right| \geq \delta_{0}(\vec{G})$, since $\left|X_{0} \cap X_{1}\right|=0$, and since a directed path $x_{0} \rightarrow v \rightarrow x_{1}$ with 
$x_{0} \in X_{0}$ and $x_{1} \in X_{1}$ would give a directed 4-cycle $\vec{C}_{4}$ containing $x$. Fact 4.2 guarantees independent sets $I_{x} \subseteq X_{1} \backslash X_{0}=X_{1}=N_{\vec{G}}^{-}(x)$ and $I_{y} \subseteq Y_{0} \backslash Y_{1}=Y_{0}=N_{\vec{G}}^{+}(y)$ of respective sizes $\left|I_{x}\right| \geq\left|N_{\vec{G}}^{-}(x)\right|-7 \varepsilon n$ and $\left|I_{y}\right| \geq\left|N_{\vec{G}}^{+}(y)\right|-7 \varepsilon n$. Note that $\left|I_{x} \cap I_{y}\right|$ is bounded by (55), we but claim that $I_{x} \cap I_{y}=\emptyset$. Indeed, a vertex $v \in I_{x} \cap I_{y}$ must have its neighborhood $N_{\vec{G}}(v)=N_{\vec{G}}^{-}(v) \cup N_{\vec{G}}^{+}(v)$ outside of $I_{x} \cup I_{y}$, and so

$$
\begin{aligned}
\left|N_{\vec{G}}^{-}(x) \cap N_{\vec{G}}^{+}(y)\right| & \geq\left|I_{x} \cap I_{y}\right|=\left|I_{x}\right|+\left|I_{y}\right|-\left|I_{x} \cup I_{y}\right| \geq\left|I_{x}\right|+\left|I_{y}\right|+\left|N_{\vec{G}}^{+}(v)\right|+\left|N_{\vec{G}}^{-}(v)\right|-n \\
& \stackrel{\text { Fct } \frac{4.2]}{\geq}\left|N_{\vec{G}}^{-}(x)\right|-7 \varepsilon n+}{ }+\left|N_{\vec{G}}^{+}(y)\right|-7 \varepsilon n+\left|N_{\vec{G}}^{+}(v)\right|+\left|N_{\vec{G}}^{-}(v)\right|-n \\
& \geq 4 \delta_{0}(\vec{G})-14 \varepsilon n-n \geq 4\left(\frac{1}{3}-\varepsilon\right) n-14 \varepsilon-n=\left(\frac{1}{3}-18 \varepsilon\right) n
\end{aligned}
$$

contradicts (55).

Second, we claim that every $a_{x} \in I_{x}$ and $b_{y} \in I_{y}$ satisfy

$$
N_{\vec{G}}^{-}\left(a_{x}\right) \cap N_{\vec{G}}^{+}\left(b_{y}\right) \neq \emptyset .
$$

Indeed, $\left(b_{y}, a_{x}\right) \notin \vec{E}$ lest $\left(x, y, b_{y}, a_{x}, x\right)$ is a directed 4-cycle containing both $x$ and $y$. Thus, $V \backslash\left(I_{x} \cup I_{y}\right)$ contains each of $N_{\vec{G}}^{-}\left(a_{x}\right)$ and $N_{\vec{G}}^{+}\left(b_{y}\right)$, and hence their union. As such,

$$
\begin{aligned}
& \left|N_{\vec{G}}^{-}\left(a_{x}\right) \cap N_{\vec{G}}^{+}\left(b_{y}\right)\right|=\left|N_{\vec{G}}^{-}\left(a_{x}\right)\right|+\left|N_{\vec{G}}^{+}\left(b_{y}\right)\right|-\left|N_{\vec{G}}^{-}\left(a_{x}\right) \cup N_{\vec{G}}^{+}\left(b_{y}\right)\right| \geq\left|N_{\vec{G}}^{-}\left(a_{x}\right)\right|+\left|N_{\vec{G}}^{+}\left(b_{y}\right)\right|+\left|I_{x} \cup I_{y}\right|-n
\end{aligned}
$$

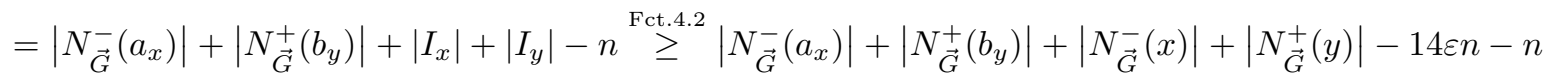

$$
\begin{aligned}
& \geq 4 \delta_{0}(\vec{G})-14 \varepsilon n-n \geq 4\left(\frac{1}{3}-\varepsilon\right) n-14 \varepsilon n-n=\left(\frac{1}{3}-18 \varepsilon\right) n>0 .
\end{aligned}
$$

Third and finally, we observe that $N_{\vec{G}}^{+}(x) \cap I_{y}=\emptyset=N_{\vec{G}}^{-}(y) \cap I_{x}$. Indeed, and for example, any $b_{y} \in N_{\vec{G}}^{+}(x) \cap I_{y}$ and $a_{x} \in I_{x}$ beget $c_{x y} \in N_{\vec{G}}^{-}\left(a_{x}\right) \cap N_{\vec{G}}^{+}\left(b_{y}\right)$ by (57), in which case $\left(x, b_{y}, c_{x y}, a_{x}, x\right)$ would be a directed 4-cycle containing $x$. Now, $V \backslash\left(I_{x} \cup I_{y}\right)$ contains each of $N_{\vec{G}}^{+}(x)$ and $N_{\vec{G}}^{-}(y)$, and so

$$
\left|N_{\vec{G}}^{+}(x) \cap N_{\vec{G}}^{-}(y)\right| \geq\left|N_{\vec{G}}^{+}(x)\right|+\left|N_{\vec{G}}^{-}(y)\right|+\left|I_{x} \cup I_{y}\right|-n,
$$

where calculations identical to (58) establish (56).

Proof of Fact 4.6. Let $\lambda>0$ be given. The promised constant $\varepsilon=\varepsilon(\lambda)>0$ will be defined in context. Let $\vec{G}=(V, \vec{E})$ be an oriented graph on $n \geq n_{0}(\lambda, \varepsilon)$ many vertices which satisfies $\delta_{0}(\vec{G}) \geq((1 / 3)-\varepsilon) n$. We show that $\vec{G}$ is $\lambda$-extremal when Conditions (1) or (2) hold, which we handle separately.

For Condition (1), it suffices to take $\varepsilon \in(0, \lambda / 7]$. Let $V=V_{0} \cup V_{1} \cup V_{2}$ be a partition satisfying $\left|V_{1}\right|,\left|V_{2}\right| \geq((1 / 3)-\varepsilon) n$ and $e_{\vec{G}}\left(V_{1}\right), e_{\vec{G}}\left(V_{2}\right), e_{\vec{G}}\left(V_{2}, V_{1}\right) \leq \varepsilon n^{2}$. We bound each of $e_{\vec{G}}\left(V_{2}, V_{0}\right), e_{\vec{G}}\left(V_{0}, V_{1}\right)$, and $e_{\vec{G}}\left(V_{1}, V_{2}\right)$ suitably from below. First, our hypotheses give

$$
e_{\vec{G}}\left(V_{2}, V_{0}\right) \geq\left(\sum_{v_{2} \in V_{2}} \operatorname{deg}_{\vec{G}}^{+}\left(v_{2}\right)\right)-e_{\vec{G}}\left(V_{2}\right)-e_{\vec{G}}\left(V_{2}, V_{1}\right) \geq\left(\frac{1}{3}-\varepsilon\right)^{2} n^{2}-2 \varepsilon n^{2} \geq\left(\frac{1}{9}-\lambda\right) n^{2},
$$

where we used $3 \varepsilon \leq \lambda$. Second, and similarly,

$$
e_{\vec{G}}\left(V_{0}, V_{1}\right) \geq\left(\sum_{v_{1} \in V_{1}} \operatorname{deg}_{\vec{G}}^{-}\left(v_{1}\right)\right)-e_{\vec{G}}\left(V_{1}\right)-e_{\vec{G}}\left(V_{2}, V_{1}\right) \geq\left(\frac{1}{3}-\varepsilon\right)^{2} n^{2}-2 \varepsilon n^{2} \geq\left(\frac{1}{9}-\lambda\right) n^{2},
$$

where we again used $3 \varepsilon \leq \lambda$. Note that, since $\vec{G}$ is oriented, our hypotheses and (59) give

$$
e_{\vec{G}}\left(V_{0}, V_{2}\right) \leq\left|V_{0}\right|\left|V_{2}\right|-e_{\vec{G}}\left(V_{2}, V_{0}\right) \leq\left(\frac{n-\left|V_{1}\right|}{2}\right)^{2}-e_{\vec{G}}\left(V_{2}, V_{0}\right) \leq 5 \varepsilon n^{2} .
$$

Third, our hypotheses and (60) give

$$
e_{\vec{G}}\left(V_{1}, V_{2}\right) \geq\left(\sum_{v_{2} \in V} \operatorname{deg}_{\vec{G}}^{-}\left(v_{2}\right)\right)-e_{\vec{G}}\left(V_{2}\right)-e_{\vec{G}}\left(V_{0}, V_{2}\right) \geq\left(\frac{1}{3}-\varepsilon\right)^{2} n^{2}-6 \varepsilon n^{2} \geq\left(\frac{1}{9}-\lambda\right) n^{2},
$$

where we used $7 \varepsilon \leq \lambda$. 
For Condition (2), we consider a suitably small $\gamma \in(0, \lambda / 3]$ in context, and we take $\varepsilon=\varepsilon(\gamma)>0$ according to an application of the Erdős-Stone theorem [6], discussed below. Assume that $\vec{G}=(V, \vec{E})$ has no transitive triangles. Then the underlying graph $G=(V, E)$ (obtained by removing orientations on $\operatorname{arcs}$ ) is $K_{4}$-free. By our hypothesis,

$$
|\vec{E}|=\sum_{v \in V} \operatorname{deg}_{\vec{G}}^{+}(v) \geq n \delta_{0}(\vec{G}) \geq\left(\frac{1}{3}-\varepsilon\right) n^{2},
$$

and so altogether the underlying graph $G=(V, E)$ is $K_{4}$-free with $|E| \geq((1 / 3)-\varepsilon) n^{2}$ many edges. As such, the Erdős-Stone theorem [6] guarantees a partition $V=V_{0} \cup V_{1} \cup V_{2}$, where $\left|V_{0}\right| \leq\left|V_{1}\right| \leq\left|V_{2}\right| \leq$ $\left|V_{0}\right|+1$, and where each $0 \leq i<j \leq 2$ satisfies

$$
\left|E\left(G\left[V_{i}, V_{j}\right]\right)\right| \geq\left(\frac{1}{9}-\gamma\right) n^{2} .
$$

Then2, the 3-partite graph

$$
G\left[V_{0}, V_{1}\right] \cup G\left[V_{1}, V_{2}\right] \cup G\left[V_{2}, V_{0}\right] \text { admits at least }((1 /(27))-\mu) n^{3} \text { many triangles } K_{3},
$$

where $\mu=\mu(\gamma) \rightarrow 0$ as $\gamma \rightarrow 0$. Since $\vec{G}$ has no transitive triangles, every triangle of $G$ corresponds to a directed 3-cycle $\vec{C}_{3}$ in $\vec{G}$. Among other conclusions, we will show that almost all of the triangles of (62) are commonly oriented, in one of the following two senses. We say that a directed 3-cycle $\vec{C}_{3}$ of $\vec{G}$ is positively oriented when all of its arcs are among $\left(V_{0} \times V_{1}\right) \cup\left(V_{1} \times V_{2}\right) \cup\left(V_{2} \times V_{0}\right)$, and we say that it is negatively oriented when all of its arcs are among $\left(V_{0} \times V_{2}\right) \cup\left(V_{2} \times V_{1}\right) \cup\left(V_{1} \times V_{0}\right)$.

We average (62) over, say $V_{0}$, to obtain a vertex $\bar{v}_{0} \in V_{0}$ belonging to at least $((1 / 9)-2 \mu) n^{2}$ many directed 3 -cycles $\vec{C}_{3}$ of $\vec{G}$. At least half of these directed 3-cycles are commonly oriented, so w.l.o.g. assume that at least half are positively oriented. Then

$\bar{v}_{0} \in V_{0}$ belongs to at least $((1 / 9)-2 \mu) n^{2}$ many directed 3-cycles $\vec{C}_{3}$ of $\vec{G}$, and in particular $\bar{v}_{0} \in V_{0}$ belongs to at least $((1 /(18))-\mu) n^{2}$ many positively oriented 3-cycles $\vec{C}_{3}$ of $\vec{G}$.

From (63), we will prove that

$$
e_{\vec{G}}\left(V_{1}, V_{2}\right) \geq\left(\frac{1}{9}-\lambda\right) n^{2}
$$

follows. Indeed, $\vec{G}$ has no transitive triangles, so each of

$$
K\left[N_{\vec{G}}^{+}\left(\bar{v}_{0}\right) \cap V_{1}, N_{\vec{G}}^{+}\left(\bar{v}_{0}\right) \cap V_{2}\right] \quad \text { and } \quad K\left[N_{\vec{G}}^{-}\left(\bar{v}_{0}\right) \cap V_{1}, N_{\vec{G}}^{-}\left(\bar{v}_{0}\right) \cap V_{2}\right]
$$

is edge-disjoint from $E$. Consequently, (61) gives that each has size at most

$$
\begin{aligned}
&\left|V_{1}\right|\left|V_{2}\right|-\left(\frac{1}{9}-\gamma\right) n^{2} \leq\left(\frac{n-\left|V_{0}\right|}{2}\right)^{2}-\left(\frac{1}{9}-\gamma\right) n^{2} \leq 2 \gamma n^{2} \\
& \Longrightarrow \quad\left|N_{\vec{G}}^{+}\left(\bar{v}_{0}\right) \cap V_{1}\right| \leq \sqrt{2 \gamma} n \quad \text { or } \quad\left|N_{\vec{G}}^{+}\left(\bar{v}_{0}\right) \cap V_{2}\right| \leq \sqrt{2 \gamma} n, \\
& \text { and }\left|N_{\vec{G}}^{-}\left(\bar{v}_{0}\right) \cap V_{1}\right| \leq \sqrt{2 \gamma} n \quad \text { or } \quad\left|N_{\vec{G}}^{-}\left(\bar{v}_{0}\right) \cap V_{2}\right| \leq \sqrt{2 \gamma} n .
\end{aligned}
$$

By (63), it must be that both $\left|N_{\vec{G}}^{+}\left(\bar{v}_{0}\right) \cap V_{2}\right| \leq \sqrt{2 \gamma} n$ and $\left|N_{\vec{G}}^{-}\left(\bar{v}_{0}\right) \cap V_{1}\right| \leq \sqrt{2 \gamma} n$ hold. As such, $\bar{v}_{0} \in V_{0}$ belongs to at most $2 \gamma n^{2}$ many negatively oriented triangles, and so (63) may be updated to say that $\bar{v}_{0} \in V_{0}$ belongs to at least $((1 / 9)-2 \mu-2 \gamma) n^{2}$ many positively oriented triangles. As such,

$$
e_{\vec{G}}\left(V_{1}, V_{2}\right) \geq\left(\frac{1}{9}-2 \mu-2 \gamma\right) n^{2} \geq\left(\frac{1}{9}-\lambda\right) n^{2}
$$

holds by taking $2 \mu+2 \gamma \leq \lambda$, and renders (64).

The argument above shows that, for each $i \in \mathbb{Z}_{3}$,

$\left(a_{i}\right)$ either $e_{\vec{G}}\left(V_{i}, V_{i+1}\right) \geq((1 / 9)-\lambda) n^{2}$,

$\left(b_{i}\right)$ or $e_{\vec{G}}\left(V_{i+1}, V_{i}\right) \geq((1 / 9)-\lambda) n^{2}$.

\footnotetext{
${ }^{2}$ See, for example, the proof of Fact 2.5
} 
These outcomes must be consistent across $i \in \mathbb{Z}_{3}$, which is to say that either $\left(a_{0}\right),\left(a_{1}\right)$, and $\left(a_{2}\right)$ all hold, or $\left(b_{0}\right),\left(b_{1}\right)$, and $\left(b_{2}\right)$ all hold. Indeed, assuming otherwise $\vec{G}$ would have $\Omega\left(n^{3}\right)$ many transitive triangles, contradicting our hypothesis. This proves that $\vec{G}$ is $\lambda$-extremal, as desired.

\section{Proof of Lemma 2.9 - Part 1: Strategy and Coarse Structure}

It suffices to take the promised constant $\lambda_{0}>0$ as

$$
\lambda_{0}=\left(\frac{1}{32,000}\right)^{4} .
$$

Now, fix $0<\lambda \leq \lambda_{0}$ and fix an integer $\ell \geq 4$ which is not divisible by three. In all that follows, we take the integer $n_{0}=n_{0}\left(\lambda_{0}, \lambda, \ell\right)$ to be sufficiently large whenever needed. The proof of Lemma 2.9 is fairly technical, so we begin by outlining some of its strategy.

6.1. Initial strategy. Let $(G, c)$ be a $\lambda$-extremal edge-colored graph on $n \geq n_{0}$ many vertices. Recall that the hypotheses in Statements (1) and (2) of Lemma 2.9 assume

$$
\delta^{c}(G) \geq \begin{cases}(n+5) / 3 & \text { in Statement }(1), \\ (n+4) / 3 & \text { in Statement (2). }\end{cases}
$$

If $(G, c)$ admits a rainbow $\ell$-cycle $C_{\ell}$, then the conclusions of Lemma 2.9 hold, so

$$
\text { we assume throughout this proof that }(G, c) \text { admits no rainbow } \ell \text {-cycles } C_{\ell} \text {. }
$$

Moreover,

we assume throughout this proof that $(G, c)$ is edge-minimal w.r.t. satisfying both (66) and (67).

Observe, for example, that (68) implies that $(G, c)$ admits no monochromatic paths $P$ or cycles $C$ on three or more edges, lest removing an internal edge $\{x, y\} \in E$ from $P$ or $C$ lowers neither $\operatorname{deg}_{G}^{c}(x)$ nor $\operatorname{deg}_{G}^{c}(y)$. Finally, for both cases of (66), we set $m=\lfloor n / 3\rfloor$, where

$$
\delta^{c}(G) \geq \frac{n+4}{3} \quad \Longrightarrow \quad \delta^{c}(G) \geq\left\lfloor\frac{n}{3}\right\rfloor+2=m+2 .
$$

Since we assume in Lemma 2.9 that $(G, c)$ is $\lambda$-extremal, fix a $\lambda$-extremal partition $V=V(G)=$ $V_{0} \cup V_{1} \cup V_{2}$ of $(G, c)$ (recall Definition [2.4). Our first main goal in proving Lemma 2.9 is to infer from (68) that $(G, c)$ enjoys nearly cannonical structure on $V_{0} \cup V_{1} \cup V_{2}$, in the following sense. Let $H=K\left[V_{0}, V_{1}, V_{2}\right]$ be the complete 3-partite graph with vertex partition $V_{0} \cup V_{1} \cup V_{2}$, and consider an edge-coloring $\kappa$ on $H$ where, for each $i \in \mathbb{Z}_{3}$ and for each $v_{i} \in V_{i}$,

(a) $\kappa$ assigns all distinct colors to the edges $\left\{v_{i}, v_{i+1}\right\} \in E$, where $v_{i+1} \in V_{i+1}$;

(b) $\kappa$ assigns a common color to all the edges $\left\{v_{i}, v_{i-1}\right\} \in E$, where $v_{i-1} \in V_{i-1}$.

We say that any such $(H, \kappa)$ is cannonical w.r.t. $V_{0} \cup V_{1} \cup V_{2}$. (For example, a cannonical edge-colored graph $(H, \kappa)$ was used in Case 1 of Section 1.1, where $\kappa=c_{+}$was defined in (2).) In the immediate sequel, we use (68) to prove that $(G, c)$ is nearly cannonical w.r.t. $V_{0} \cup V_{1} \cup V_{2}$, in the sense that for each $i \in \mathbb{Z}_{3}$, almost all $v_{i} \in V_{i}$ admit distinctly colored edges to almost all $v_{i+1} \in V_{i+1}$, and almost all $v_{i} \in V_{i}$ admit commonly colored edges to almost all $v_{i-1} \in V_{i-1}$. We now make these details precise.

6.2. $(\boldsymbol{G}, \boldsymbol{c})$ is nearly cannonical: getting started. Definition 2.4 ensures that each $i \in \mathbb{Z}_{3}$ satisfies

$$
\left|V_{i}\right|=\left(\frac{1}{3} \pm 3 \sqrt{\lambda}\right) n .
$$

Indeed, $G$ has at least $(1 / 3-3 \lambda) n^{2}$ many edges, so $\left(\begin{array}{c}V_{0} \\ 2\end{array}\right) \cup\left(\begin{array}{c}V_{1} \\ 2\end{array}\right) \cup\left(\begin{array}{c}V_{2} \\ 2\end{array}\right)$ consists of at most $((1 / 6)+3 \lambda) n^{2}$ many pairs. Set $\left|V_{i}\right|=\left((1 / 3)+e_{i}\right) n, i \in \mathbb{Z}_{3}$, so that $e_{0}+e_{1}+e_{2}=0$. Then $\left(\begin{array}{c}V_{0} \\ 2\end{array}\right) \cup\left(\begin{array}{c}V_{1} \\ 2\end{array}\right) \cup\left(\begin{array}{c}V_{2} \\ 2\end{array}\right)$ has size

$$
(1+o(1))\left(\frac{n^{2}}{6}+\frac{n^{2}}{2}\left(e_{0}^{2}+e_{1}^{2}+e_{2}^{2}\right)\right),
$$

and this is too large when $\max \left\{\left|e_{0}\right|,\left|e_{1}\right|,\left|e_{2}\right|\right\}>\sqrt{6 \lambda}$.

Next, fix $i \in \mathbb{Z}_{3}$. We shall say that a vertex $v_{i} \in V_{i}$ is an $i$-good vertex if

$$
\operatorname{deg}_{G}^{c}\left(v_{i}, V_{i+1}\right) \geq\left|V_{i+1}\right|-\lambda^{1 / 4} n \quad \text { and } \quad \operatorname{deg}_{G}\left(v_{i}, V_{i-1}\right) \geq\left|V_{i-1}\right|-\lambda^{1 / 4} n
$$


where as usual $\operatorname{deg}_{G}\left(v_{i}, V_{i-1}\right)$ denotes the number of neighbors of $v_{i}$ in $V_{i-1}$, and where here $\operatorname{deg}_{G}^{c}\left(v_{i}, V_{i+1}\right)$ denotes the number of colors seen on the edges of $v_{i}$ to $V_{i+1}$. Then (71) says an $i$-good vertex $v_{i}$ admits distinctly colored edges to all but $\lambda^{1 / 4} n$ many vertices $v_{i+1} \in V_{i+1}$, and it admits edges of varying colors to all but $\lambda^{1 / 4} n$ many vertices $v_{i-1} \in V_{i-1}$. Let $V_{i}^{\text {good }}$ denote the set of $i$-good vertices $v_{i} \in V_{i}$. Using (70) and Definition 2.4 it is easy to show that

$$
\left|V_{i}^{\text {good }}\right| \geq\left|V_{i}\right|-24 \lambda^{1 / 4} n
$$

With $i \in \mathbb{Z}_{3}$ still fixed, we shall say that a vertex $v_{i} \in V_{i} \backslash V_{i}^{\text {good }}$ is an $i$-bad vertex. We write $V_{i}^{\text {bad }}=$ $V_{i} \backslash V_{i}^{\text {good }}$ for the set of $i$-bad vertices, and we write $V^{\text {bad }}=V_{0}^{\text {bad }} \cup V_{1}^{\text {bad }} \cup V_{2}^{\text {bad }}$ for the set of bad vertices. Then bad vertices total at most $72 \lambda^{1 / 4} n$ by (72).

We now alter the partition $V=V_{0} \cup V_{1} \cup V_{2}$ to $V=U_{0} \cup U_{1} \cup U_{2}$, as follows. For each $i$-good vertex $v_{i} \in V_{i}^{\text {good }}$, we put $v_{i} \in U_{i}$. For each bad vertex $v \in V^{\text {bad }}$, let $j_{v} \in \mathbb{Z}_{3}$ achieve

$$
\operatorname{deg}_{G}^{c}\left(v_{i}, V_{j_{v}}^{\text {good }}\right)=\max \left\{\operatorname{deg}_{G}^{c}\left(v, V_{0}^{\text {good }}\right), \operatorname{deg}_{G}^{c}\left(v, V_{1}^{\text {good }}\right), \operatorname{deg}_{G}^{c}\left(v, V_{2}^{\text {good }}\right)\right\} .
$$

We then put $v \in U_{j_{v}-1}$. Then $U_{i}$ consists of $V_{i}^{\text {good }}$ together with those bad vertices $v \in V^{\text {bad }}$ satisfying

$$
\operatorname{deg}_{G}^{c}\left(v, V_{i+1}^{\text {good }}\right) \geq \max \left\{\operatorname{deg}_{G}^{c}\left(v, V_{i}^{\text {good }}\right), \operatorname{deg}_{G}^{c}\left(v, V_{i-1}^{\text {good }}\right)\right\} .
$$

We write $U_{i}^{\text {good }}=U_{i} \cap V_{i}^{\text {good }}=V_{i}^{\text {good }}$, and we maintain that these vertices are good. We write $U_{i}^{\text {bad }}=U_{i} \cap V^{\text {bad }}$, and we maintain that these vertices are bad. Then (70)-(72) give:

$$
\begin{gathered}
\left|U_{i}\right|=\left(\frac{1}{3} \pm 75 \lambda^{1 / 4}\right) n, \quad\left|U_{i}^{\text {good }}\right|=\left(\frac{1}{3} \pm 75 \lambda^{1 / 4}\right) n, \quad\left|V^{\mathrm{bad}}\right|=\left|U_{0}^{\mathrm{bad}}\right|+\left|U_{1}^{\mathrm{bad}}\right|+\left|U_{2}^{\mathrm{bad}}\right| \leq 72 \lambda^{1 / 4} n, \\
\forall i \in \mathbb{Z}_{3}, \forall u \in U_{i}^{\text {good }}, \operatorname{deg}_{G}^{c}\left(u, U_{i+1}\right) \geq\left|V_{i+1}\right|-73 \lambda^{1 / 4} n \geq\left|U_{i+1}\right|-145 \lambda^{1 / 4} n, \\
\forall i \in \mathbb{Z}_{3}, \forall u \in U_{i}^{\text {good }}, \operatorname{deg}_{G}^{c}\left(u, U_{i+1}^{\text {good }}\right) \geq\left(\frac{1}{3}-76 \lambda^{1 / 4}\right) n, \\
\forall i \in \mathbb{Z}_{3}, \forall u \in U_{i}^{\text {good }}, \operatorname{deg}_{G}\left(u, U_{i-1}\right) \geq\left|V_{i-1}\right|-73 \lambda^{1 / 4} n \geq\left|U_{i-1}\right|-145 \lambda^{1 / 4} n, \\
\forall i \in \mathbb{Z}_{3}, \forall u \in U_{i}^{\text {good }}, \operatorname{deg}_{G}\left(u, U_{i-1}^{\text {good }}\right) \geq\left(\frac{1}{3}-76 \lambda^{1 / 4}\right) n, \\
\forall i \in \mathbb{Z}_{3}, \forall u \in U_{i}^{\text {bad }}, \operatorname{deg}_{G}^{c}\left(u, U_{i+1}\right) \stackrel{(73)}{\geq} \frac{1}{3} \delta^{c}(G)-72 \lambda^{1 / 4} n \stackrel{\left(\frac{69}{9}\right)}{\geq}\left(\frac{1}{9}-72 \lambda^{1 / 4}\right) n .
\end{gathered}
$$

Henceforth, the initial partition $V=V_{0} \cup V_{1} \cup V_{2}$ is largely usurped by $V=U_{0} \cup U_{1} \cup U_{2}$.

6.3. $(\boldsymbol{G}, \boldsymbol{c})$ is nearly cannonical: a next step. The inequalities in (75) show that $\left|U_{0}\right|,\left|U_{1}\right|,\left|U_{2}\right|$ are nearly balanced, and that $G\left[U_{0}, U_{1}, U_{3}\right]$ differs from the complete 3-partite graph $K\left[U_{0}, U_{1}, U_{2}\right]$ on few edges. The inequalities in (75) also show that $(G, c)$ deviates very little from property $(a)$ of Section 6.1, in that good vertices $u_{i} \in U_{i}^{\text {good }}$ (which are pervasive) have distinctly colored edges to nearly all $u_{i+1} \in U_{i+1}$. We now show that $(G, c)$ deviates little from the corresponding property $(b)$. For that, we first show that good vertices $u_{i} \in U_{i}^{\text {good }}$ are incident to few colors $c\left(\left\{u_{i}, u_{i-1}\right\}\right)$, where $u_{i-1} \in U_{i-1}^{\text {good }}$.

Fact 6.1. For each $i \in \mathbb{Z}_{3}$ and for each $u_{i} \in U_{i}^{\text {good }}$, we have $\operatorname{deg}_{G}^{c}\left(u_{i}, U_{i-1}^{\text {good }}\right) \leq 160 \lambda^{1 / 4} n$.

Proof of Fact 6.1. Assume for contradiction that Fact 6.1 is false for some index $i \in \mathbb{Z}_{3}$ and vertex $u_{i} \in U_{i}^{\text {good }}$, and w.l.o.g. assume $i=2$. We will first determine a set $T_{1}^{\text {good }} \subseteq U_{1}^{\text {good }}$ so that the fixed good vertex $u_{2} \in U_{2}^{\text {good }}$ satisfies

$$
\operatorname{deg}_{G}^{c}\left(u_{2}, T_{1}^{\text {good }}\right)>0 \text { and every path }\left(u_{2}, u_{1}, v\right) \text { in } G \text { where } u_{1} \in T_{1}^{\text {good }} \text { is rainbow. }
$$

To prove (76), we distinguish $\operatorname{deg}_{G}^{c}\left(u_{2}\right)$.

Case $1\left(\operatorname{deg}_{G}^{c}\left(u_{2}\right) \geq(n+10) / 3\right)$. Set $T_{1}^{\text {good }}=U_{1}^{\text {good }}$, where our contrary assumption gives

$$
\operatorname{deg}_{G}^{c}\left(u_{2}, T_{1}^{\text {good }}\right)=\operatorname{deg}_{G}^{c}\left(u_{2}, U_{1}^{\text {good }}\right)>160 \lambda^{1 / 4} n>0,
$$


as desired. Now, every path $\left(u_{2}, u_{1}, v\right)$ with $u_{1} \in T_{1}^{\text {good }}=U_{1}^{\text {good }}$ is rainbow lest the hypothesis of Case 1 gives that removing the edge $\left\{u_{2}, u_{1}\right\} \in E$ from $G$ contradicts (68).

Case $2\left(\operatorname{deg}_{G}^{c}\left(u_{2}\right)<(n+10) / 3\right)$. Set

$$
T_{1}^{\text {good }}=\left\{u_{1} \in U_{1}^{\text {good }}: \exists u_{0} \in U_{0} \text { where } c\left(\left\{u_{2}, u_{0}\right\}\right)=c\left(\left\{u_{2}, u_{1}\right\}\right)\right\} .
$$

Observe that

and so

$$
\operatorname{deg}_{G}^{c}\left(u_{2}\right) \geq \operatorname{deg}_{G}^{c}\left(u_{2}, U_{0}\right)+\operatorname{deg}_{G}^{c}\left(u_{2}, U_{1}^{\text {good }}\right)-\operatorname{deg}_{G}^{c}\left(u_{2}, T_{1}^{\text {good }}\right),
$$

$$
\begin{aligned}
& \operatorname{deg}_{G}^{c}\left(u_{2}, T_{1}^{\text {good }}\right) \geq \operatorname{deg}_{G}^{c}\left(u_{2}, U_{0}\right)+\operatorname{deg}_{G}^{c}\left(u_{2}, U_{1}^{\text {good }}\right)-\operatorname{deg}_{G}^{c}\left(u_{2}\right) \\
& \stackrel{755}{\geq}\left(\frac{1}{3}-76 \lambda^{1 / 4}\right) n+\operatorname{deg}_{G}^{c}\left(u_{2}, U_{1}^{\text {good }}\right)-\operatorname{deg}_{G}^{c}\left(u_{2}\right) \stackrel{\sqrt{77}}{>}\left(\frac{1}{3}-76 \lambda^{1 / 4}\right) n+160 \lambda^{1 / 4} n-\operatorname{deg}_{G}^{c}\left(u_{2}\right) \\
& \stackrel{\text { Case 2 }}{>}\left(\frac{1}{3}-76 \lambda^{1 / 4}\right) n+160 \lambda^{1 / 4} n-\frac{n+10}{3}=84 \lambda^{1 / 4} n-O(1)>0 .
\end{aligned}
$$

If $\left(u_{2}, u_{1}, v\right)$ is a monochromatic path with $u_{1} \in T_{1}^{\text {good }}$, then there exists $u_{0} \in U_{0}$ where $\left(u_{0}, u_{2}, u_{1}, v\right)$ is monochromatic. Whether or not $v=u_{0}$, removing the edge $\left\{u_{2}, u_{1}\right\} \in E$ from $G$ contradicts (68).

We now use (76) to complete the proof of Fact 6.1. Fix an arbitrary vertex $u_{1} \in T_{1}^{\text {good }}$ (cf. (76)), and fix an arbitrary vertex $u_{0} \in N_{G}\left(u_{1}, U_{0}^{\text {good }}\right)$ (cf. (75)). By (76), the path $\left(u_{2}, u_{1}, u_{0}\right)$ is rainbow. We now distinguish the cases $\ell \equiv 1,2(\bmod 3)$.

Case A $(\ell \equiv 1(\bmod 3))$. Using (75) and $n \geq n_{0}(\ell)$ sufficiently large, we can easily extend the rainbow path $\left(u_{2}, u_{1}, u_{0}\right)$ to a rainbow path $R_{\ell-4}=\left(u_{2}, u_{1}, u_{0}, v_{1}, v_{2}, \ldots, v_{\ell-4}\right)$ on $\ell-1$ vertices, where for each $1 \leq j \leq \ell-4$, we may choose $v_{j} \in U_{J}^{\text {good }}$ for $J \equiv j(\bmod 3)$. Our contrary assumption gives that $u_{2} \in U_{2}^{\text {good }}$ sees $160 \lambda^{1 / 4} n$ colors into $U_{1}^{\text {good }}$, and at most $\ell-1$ of them were used on $R_{\ell-4}$. Similarly, (75) gives that the vertex $v_{\ell-4} \in U_{0}^{\text {good }}($ recall $\ell \equiv 1(\bmod 3))$ sees $\left((1 / 3)-76 \lambda^{1 / 4}\right) n$ colors into $U_{1}^{\text {good }}$, and at most $\ell-1$ of them were used on $R_{\ell-4}$. Then some $w_{1} \in N_{G}\left(u_{2}, U_{1}^{\text {good }}\right) \cap N_{G}\left(v_{\ell-4}, U_{1}^{\text {good }}\right)$ extends $R_{\ell-4}$ to a rainbow $\ell$-cycle $C_{\ell}$ as

$$
160 \gamma^{1 / 4} n-(\ell-1)+\left(\frac{1}{3}-76 \lambda^{1 / 4}\right) n-(\ell-1) \geq\left(\frac{1}{3}+84 \lambda^{1 / 4}\right) n-O(1) \stackrel{755}{>}\left|U_{1}\right| .
$$

Case $\mathbf{B}(\ell \equiv 2(\bmod 3))$. The rainbow path $\left(u_{2}, u_{1}\right)$ may be extended to a rainbow path $\hat{R}_{\ell-2}=$ $\left(u_{2}, u_{1}, v_{2}, \ldots, v_{\ell-2}\right)$ on $\ell-1$ vertices, where for each $2 \leq j \leq \ell-2$, we may choose $v_{j} \in U_{J}^{\text {good }}$ for $J \equiv j$ (mod 3). Identically to the above, we may extend the rainbow path $\hat{R}_{\ell-2}$ to a rainbow $\ell$-cycle $C_{\ell}$.

6.4. $(\boldsymbol{G}, \boldsymbol{c})$ is nearly cannonical: finale. We now show that, for a fixed $u_{i} \in U_{i}^{\text {good }}$, edges $\left\{u_{i}, u_{i-1}\right\} \in$ $E$, where $u_{i-1} \in U_{i-1}^{\text {good }}$, are dominated by a single color.

Proposition 6.2. For each $i \in \mathbb{Z}_{3}$ and for each $u_{i} \in U_{i}^{\text {good }}$, there exists a color $c_{u_{i}}$ from $c$ where all but $161 \lambda^{1 / 4} n$ many vertices $u_{i-1} \in U_{i-1}^{\text {good }}$ satisfy $\left\{u_{i}, u_{i-1}\right\} \in E$ and $c\left(\left\{u_{i}, u_{i-1}\right\}\right)=c_{u_{i}}$. Together with (75), all but $233 \lambda^{1 / 4} n$ vertices $u_{i-1} \in U_{i-1}$ satisfy $\left\{u_{i}, u_{i-1}\right\} \in E$ and $c\left(\left\{u_{i}, u_{i-1}\right\}\right)=c_{u_{i}}$.

For the proof and use of Proposition [6.2 we establish some notation. Fix $i \in \mathbb{Z}_{3}$ and fix $u_{i} \in U_{i}$. On the edges $E_{G}\left(u_{i}, U_{i-1}\right)$ between $u_{i}$ and $U_{i-1}$, let $c_{u_{i}}$ be a most frequent color, which we call the primary color of $E_{G}\left(u_{i}, U_{i-1}\right)$. Edges of $E_{G}\left(u_{i}, U_{i-1}\right)$ colored by $c_{u_{i}}$ are called typical edges, and edges of $E_{G}\left(u_{i}, U_{i-1}\right)$ colored otherwise are called special edges. We write $N_{G}^{\text {typ }}\left(u_{i}, U_{i-1}\right)$ for the set of $u_{i-1} \in U_{i-1}$ where $\left\{u_{i}, u_{i-1}\right\} \in E$ is a typical edge, and we write $N_{G}^{\mathrm{spec}}\left(u_{i}, U_{i-1}\right)$ for the set of $u_{i-1} \in U_{i-1}$ where $\left\{u_{i}, u_{i-1}\right\} \in E$ is a special edge. We write

$$
\operatorname{deg}_{G}^{\text {typ }}\left(u_{i}, U_{i-1}\right)=\left|N_{G}^{\mathrm{typ}}\left(u_{i}, U_{i-1}\right)\right| \quad \text { and } \quad \operatorname{deg}_{G}^{\text {spec }}\left(u_{i}, U_{i-1}\right)=\left|N_{G}^{\text {spec }}\left(u_{i}, U_{i-1}\right)\right| .
$$


Proof of Proposition 6.2. Assume for contradiction that Proposition 6.2 is false for some index $i \in \mathbb{Z}_{3}$ and vertex $u_{i} \in U_{i}^{\text {good }}$, and w.l.o.g. assume $i=2$. Then, the fixed vertex $u_{2} \in U_{2}^{\text {good }}$ satisfies

$$
\operatorname{deg}_{G}^{\text {spec }}\left(u_{2}, U_{1}^{\text {good }}\right) \geq 161 \lambda^{1 / 4} n \quad \text { while } \quad \operatorname{deg}_{G}^{c}\left(u_{2}, U_{1}^{\text {good }}\right) \stackrel{\text { Fact }}{\leq} 160 \lambda^{1 / 4} n .
$$

We will produce a contradiction similar to that for Fact 6.1, where we will use (79) to construct a rainbow $\ell$-cycle $C_{\ell}$ in $(G, c)$, which will contradict (67). We again distinguish the cases $\ell \equiv 1,2(\bmod 3)$.

Case $1(\ell \equiv 1(\bmod 3))$. The inequalities in (79) together imply that there exist neighbors $u_{1} \neq v_{1} \in$ $N_{G}\left(u_{2}, U_{1}^{\text {good }}\right)$ for which $c\left(\left\{u_{2}, u_{1}\right\}\right)=c\left(\left\{u_{2}, v_{1}\right\}\right)$ differs from the primary color $c_{u_{2}}$. For simplicity, let $c_{u_{2}}$ be blue and let $c\left(\left\{u_{2}, u_{1}\right\}\right)=c\left(\left\{u_{2}, v_{1}\right\}\right)$ be red. Using (75), fix $u_{0} \neq v_{0} \in N_{G}\left(u_{1}, U_{0}^{\text {good }}\right) \cap$ $N_{G}\left(v_{1}, U_{0}^{\text {good }}\right)$. Since $(G, c)$ admits no monochromatic paths on four vertices, none of the edges of the four-cycle $\left(u_{1}, u_{0}, v_{1}, v_{0}\right)$ can be red, and not all of them can be blue. W.l.o.g., assume $\left\{u_{1}, u_{0}\right\}$ is colored yellow so that $\left(u_{2}, u_{1}, u_{0}\right)$ is a red-yellow path which avoids the primary color blue for $u_{2}$. Similarly to the proof of Fact 6.1, we will extend $\left(u_{2}, u_{1}, u_{0}\right)$ to a rainbow $\ell$-cycle $C_{\ell}$, which will contradict (67).

Consider the following set which will be an eventual 'target space':

$$
T_{1}\left(u_{2}\right)=\left\{t_{1} \in N_{G}\left(u_{2}, U_{1}^{\text {good }}\right): c\left(\left\{u_{2}, t_{1}\right\}\right) \text { is neither red nor yellow }\right\} \subseteq U_{1}^{\text {good }} .
$$

Since blue is the primary color for $u_{2}$, some edges $\left\{u_{2}, t_{1}\right\}$ with $t_{1} \in T_{1}\left(u_{2}\right)$ are colored blue. Now, among the colors blue, red, and yellow, neither red nor yellow are primary, so at most a $2 / 3$ portion of neighbors $v_{1} \in N_{G}\left(u_{2}, U_{1}^{\text {good }}\right)$ have red or yellow edges with $u_{2}$. Thus,

$$
\begin{aligned}
\left|T_{1}\left(u_{2}\right)\right| \geq \frac{1}{3} \operatorname{deg}_{G}\left(u_{2}, U_{1}^{\text {good }}\right) \stackrel{775}{\geq} \frac{1}{3}\left(\frac{1}{3}-76 \lambda^{1 / 4}\right) n \stackrel{65}{\geq} \frac{n}{10}, \\
\text { while } u_{2} \text { sees at most } 160 \lambda^{1 / 4} n \text { colors into } T_{1}\left(u_{2}\right) \text { (cf. Fact 6.1). }
\end{aligned}
$$

Let $C\left(u_{2}\right)$ be the set of colors used on edges between $u_{2}$ and $T_{1}\left(u_{2}\right)$. As we did for Fact 6.1, we extend the rainbow path $\left(u_{2}, u_{1}, u_{0}\right)$ to a rainbow path $R_{\ell-4}=\left(u_{2}, u_{1}, u_{0}, w_{1}, w_{2}, \ldots, w_{\ell-4}\right)$ on $\ell-1$ vertices, where for each $1 \leq j \leq \ell-4$, we may choose $w_{j} \in U_{J}^{\text {good }}$ for $J \equiv j(\bmod 3)$, but where this time we avoid the $\left|C\left(u_{2}\right)\right| \leq 160 \lambda^{1 / 4} n$ many colors of $C\left(u_{2}\right)$, which we may do on account of (75). Since $w_{\ell-4} \in U_{0}^{\text {good }}$ $($ recall $\ell \equiv 1(\bmod 3))$, (75) gives that $\operatorname{deg}_{G}^{c}\left(w_{\ell-4}, U_{1}^{\text {good }}\right) \geq\left|U_{1}^{\text {good }}\right|-145 \lambda^{1 / 4} n$, so from $T_{1}\left(u_{2}\right) \subseteq U_{1}^{\text {good }}$, $\operatorname{deg}_{G}^{c}\left(w_{\ell-4}, T_{1}\left(u_{2}\right)\right) \geq\left|T_{1}\left(u_{2}\right)\right|-145 \lambda^{1 / 4} n \stackrel{\frac{80}{\geq}}{\geq} \frac{n}{10}-145 \lambda^{1 / 4} n \stackrel{655}{>} 160 \lambda^{1 / 4} n+\ell-1 \stackrel{80}{\geq}\left|C\left(u_{2}\right)\right|+\ell-1$.

Thus, we may choose a neighbor $t_{1} \in N_{G}\left(w_{\ell-4}\right) \cap T_{1}\left(u_{2}\right)$ where $c\left(\left\{w_{\ell-4}, t_{1}\right\}\right) \notin C\left(u_{2}\right)$ differs from any color used on $R_{\ell-4}$. Now, $\left(u_{2}, u_{1}, u_{0}, w_{1}, w_{2}, \ldots, w_{\ell-4}, t_{1}\right)$ is a rainbow $\ell$-cycle $C_{\ell}$ in $(G, c)$ (where $c\left(\left\{u_{2}, t_{1}\right\}\right) \in C\left(u_{2}\right)$ but where $C\left(u_{2}\right)$ was used nowhere else on $\left.C_{\ell}\right)$, which contradicts (67).

Case $2(\ell \equiv 2(\bmod 3))$. The proof is analogous to that above, where we may simplify the preamble of Case 1. Here, fix a single neighbor $u_{1} \in N_{G}\left(u_{2}, U_{1}^{\text {good }}\right)$ where $c\left(\left\{u_{2}, u_{1}\right\}\right)$ (which we assume is red) differs from the primary color blue for $u_{2}$. We will extend the rainbow path $\left(u_{2}, u_{1}\right)$ to a rainbow $\ell$-cycle $C_{\ell}$, which will contradict (67). To do so, this time we define

$$
T_{1}\left(u_{2}\right)=\left\{t_{1} \in N_{G}\left(u_{2}, U_{1}^{\text {good }}\right): c\left(\left\{u_{2}, t_{1}\right\}\right) \text { is not red }\right\},
$$

and again we define $C\left(u_{2}\right)$ to be the set of colors on edges between $u_{2}$ and $T_{1}\left(u_{2}\right)$. Since red is not the primary color of $u_{2}$, at most half the neighbors $v_{1} \in N_{G}\left(u_{2}, U_{1}^{\text {good }}\right)$ have a red edge with $u_{2}$, and so the final conclusions of (80) hold. On account of (75), we may extend the rainbow path $\left(u_{2}, u_{1}\right)$ to a rainbow path $\hat{R}_{\ell-2}=\left(u_{2}, u_{1}, v_{2}, \ldots, v_{\ell-2}\right)$ on $\ell-1$ vertices, where for each $2 \leq j \leq \ell-2$, we may choose $v_{j} \in U_{J}^{\text {good }}$ for $J \equiv j(\bmod 3)$, and where again we may avoid the $\left|C\left(u_{2}\right)\right| \leq 160 \lambda^{1 / 4} n$ many colors of $C\left(u_{2}\right)$. The inequality in (81) holds for the vertex $v_{\ell-2} \in U_{0}^{\text {good }}($ recall $\ell \equiv 2(\bmod 3))$, so we may choose $t_{1} \in N_{G}\left(v_{\ell-2}\right) \cap T_{1}\left(u_{2}\right)$ where $c\left(\left\{v_{\ell-2}, t_{1}\right\}\right) \notin C\left(u_{2}\right)$ differs from any color used on $\hat{R}_{\ell-2}$. Now, $\left(u_{2}, u_{1}, v_{2}, \ldots, v_{\ell-2}, t_{1}\right)$ is a rainbow $\ell$-cycle $C_{\ell}$ in $(G, c)$, which contradicts (67). 
We conclude the nearly cannonical structure of $(G, c)$ by noting that, for each $i \in \mathbb{Z}_{3}$, distinct good vertices $u_{i} \neq v_{i} \in U_{i}^{\text {good }}$ admit distinct primary colors.

Corollary 6.3. For each $i \in \mathbb{Z}_{3}$ and for each $u_{i} \neq v_{i} \in U_{i}^{\text {good }}$, the primary colors $c_{u_{i}}$ and $c_{v_{i}}$ differ.

Proof of Corollary 6.3. Fix $i \in \mathbb{Z}_{3}$ and fix $u_{i} \neq v_{i} \in U_{i}^{\text {good }}$. Then

$$
\left|N_{G}^{\mathrm{typ}}\left(u_{i}, U_{i-1}\right) \cap N_{G}^{\mathrm{typ}}\left(v_{i}, U_{i-1}\right)\right| \stackrel{\text { Prop 6.2 }}{\geq}\left|U_{i-1}\right|-466 \lambda^{1 / 4} \stackrel{\sqrt[750]{\geq}}{\geq}\left(\frac{1}{3}-541 \lambda^{1 / 4}\right) n \stackrel{65}{\geq} 2 .
$$

If $c_{u_{i}}=c_{v_{i}}$, then any pair from the set above renders a monochromatic 4-cycle, contradicting (68).

\section{Proof of Lemma 2.9 - Part 2: Strong Cycles and the Case $\ell \equiv 2$ (mod 3 )}

Continuing from the previous section, we now prepare to prove Lemma 2.9 when $\ell \equiv 2(\bmod 3)$. The central tools of this proof are important observations on so-called strong cycles in the nearly cannonical edge-colored graph $(G, c)$. Many of these observations will also be important later when we prove the case $\ell \equiv 1(\bmod 3)$ of Lemma 2.9 .

7.1. Strong cycles. We say that a cycle $C_{k}=\left(u_{1}, \ldots, u_{k}\right)$ (with prescribed vertex $u_{1}$ ) is a strong cycle if there exists $i \in \mathbb{Z}_{3}$ so that $u_{1} \in U_{i}^{\text {good }}$ and $u_{k} \in N_{G}^{\text {typ }}\left(u_{1}, U_{i-1}\right)$. We determine conditions under which rainbow or properly colored paths can be extended to strong rainbow or strong properly colored cycles.

Proposition 7.1. Fix integers $1 \leq k<K \leq \ell$ and fix $i, j \in \mathbb{Z}_{3}$ for which $K-k \equiv(i-1)-j(\bmod 3)$. Let $P$ be $a\left(u_{i}, u_{j}\right)$-path on $k$ vertices linking $u_{i} \in U_{i}^{\text {good }}$ and $u_{j} \in U_{j}$. The following statements hold:

(1) If $P$ is rainbow and $c_{u_{i}}$-free, then $P$ may be extended to a strong rainbow $K$-cycle $C_{K}$;

(2) If $P$ is properly colored and its $u_{i}$-edge is not $c_{u_{i}}$-colored, then $P$ may be extended to a strong properly colored $K$-cycle $C_{K}$;

(3) When $K \equiv k(\bmod 3)$ and $(G, c)$ admits a strong rainbow $k$-cycle $C_{k}$, then $(G, c)$ also admits a strong rainbow $K$-cycle $C_{K}$;

(4) When $K \equiv k(\bmod 3)$ and $(G, c)$ admits a strong properly colored $k$-cycle $C_{k}$, then $(G, c)$ also admits a strong properly colored $K$-cycle $C_{K}$.

Proof of Proposition 7.1. Let integers $1 \leq k<K \leq \ell$ and elements $i, j \in \mathbb{Z}_{3}$ be given satisfying $K-k \equiv$ $(i-1)-j(\bmod 3)$, and let $P=\left(u_{i}, \ldots, u_{j}\right)$ be a $\left(u_{i}, u_{j}\right)$-path on $k$ vertices linking $u_{i} \in U_{i}^{\text {good }}$ and $u_{j} \in U_{j}$. To prove Statement (1), assume that $P=R$ is rainbow and $c_{u_{i}}$-free. Similarly to the proofs of Fact 6.1 and Proposition [6.2, we will extend $R$ to a $c_{u_{i}}$ free rainbow path $\tilde{R}_{K-k-1}=$ $\left(u_{i}, \ldots, u_{j}, v_{j+1}, \ldots, v_{j+K-k-1}\right)$ on $K-1$ vertices, where for each $j+1 \leq h \leq j+K-k-1$, we may choose $v_{h} \in U_{H}^{\text {good }}$ for $H \equiv h(\bmod 3)$. We begin with the first step, where it is not guaranteed in our hypothesis that $u_{j} \in U_{j}$ is a good vertex. If $u_{j} \in U_{j}^{\mathrm{bad}}$, then

$$
\operatorname{deg}_{G}^{c}\left(u_{j}, U_{j+1}\right) \stackrel{\sqrt[75]{7}}{\geq}\left(\frac{1}{9}-72 \lambda^{1 / 4}\right) n \quad \stackrel{\underline{\underline{75}}}{\Longrightarrow} \quad \operatorname{deg}_{G}^{c}\left(u_{j}, U_{j+1}^{\text {good }}\right) \stackrel{75}{\geq}\left(\frac{1}{9}-144 \lambda^{1 / 4}\right) n .
$$

Thus, we may select $v_{j+1} \in N_{G}\left(u_{j}, U_{j+1}^{\text {good }}\right)$ for $\tilde{R}_{K-k-1}$ while avoiding $c_{u_{i}}$ and the colors of $R$. If $u_{j} \in U_{j}^{\text {good }}$ is a good vertex, then the neighborhood $N_{G}\left(u_{j}, U_{j+1}^{\text {good }}\right)$ is larger still (cf. (75)), and again we may select $v_{j+1}$ for $\tilde{R}_{K-k-1}$ as described above. We select all remaining vertices $v_{h}$ for $\tilde{R}_{K-k-1}$, where $j+2 \leq h \leq j+K-k-1$, in a similar fashion. By our hypothesis $K-k \equiv(i-1)-j(\bmod 3)$, the terminal vertex $v_{j+K-k-1} \in U_{i-2}^{\text {good }}$ while the initial vertex $u_{i} \in U_{i}^{\text {good }}$. Comparing Proposition 6.2 and (75), we see

$$
\left|N_{G}^{\text {typ }}\left(u_{i}, U_{i-1}\right) \cap N_{G}\left(v_{j+K-k-1}, U_{i-1}\right)\right| \geq\left|U_{i-1}\right|-306 \lambda^{1 / 4} n \stackrel{\text { 75 }}{\geq}\left(\frac{1}{3}-381 \lambda^{1 / 4}\right) n \stackrel{665}{>} 0,
$$

and so we may select a vertex $u_{i-1}$ from the set above whose adjacency with $v_{j+K-k-1}$ avoids $c_{u_{i}}$ and the colors of $\tilde{R}_{K-k-1}$. Since $c\left(\left\{u_{i}, u_{i-1}\right\}\right)=c_{u_{i}}$ is the primary color of $u_{i}$, which hasn't yet been used, $C_{K}=\left(u_{i}, \ldots, u_{j}, v_{j+1}, \ldots, v_{j+K-k-1}, u_{i-1}\right)$ is a strong rainbow $K$-cycle. 
The proof of Statement (2) is absolutely the same as that of Statement (1). In particular, for the properly colored $k$-vertex path $P=\left(u_{i}, \ldots, u_{j}\right)$ linking $u_{i} \in U_{i}^{\text {good }}$ and $u_{j} \in U_{j}$ whose $u_{i}$-edge is not $c_{u_{i}}{ }^{-}$ colored, the proof above allows the segment $\left(u_{j}, v_{j+1}, \ldots, v_{j+K-k-1}, u_{i-1}\right)$ of $\tilde{R}_{K-k-1}$ to be rainbow, $c_{u_{i}}$-free, and to be free of the colors from $P$. Thus, $C_{K}=\left(u_{i}, \ldots, u_{j}, v_{j+1}, \ldots, v_{j+K-k-1}, u_{i-1}\right)$ is a strong properly colored $K$-cycle.

Statements (3) and (4) now follow immediately from Statements (1) and (2). Indeed, let $C_{k}=$ $\left(u_{1}, \ldots, u_{k}\right)$ be a strong rainbow or properly colored $k$-cycle where $u_{1} \in U_{i}^{\text {good }}$ and $u_{k} \in N^{\text {typ }}\left(u_{1}, U_{i-1}\right)$ for some $i \in \mathbb{Z}_{3}$. Ignoring the edge $\left\{u_{1}, u_{k}\right\}$, the path $P_{k}=\left(u_{1}, \ldots, u_{k}\right)$ is rainbow or properly colored, where $u_{k} \in U_{i-1}$ assumes $j=i-1$. Taking $K \equiv k+(i-1)-(i-1) \equiv k(\bmod 3)$ and $K \leq \ell$, Statements $(1)$ or $(2)$ extend $P_{k}$ to a strong rainbow or properly colored $K$-cycle $C_{K}$.

It will be convenient to have the following corollary of Proposition 7.1 in the case $\ell \equiv 2(\bmod 3)$.

Corollary 7.2. Let $\ell \equiv 2(\bmod 3)$ and fix $i \in \mathbb{Z}_{3}$. The following statements hold:

(1) Each $u_{i} \in U_{i}^{\text {good }}$ satisfies $N_{G}^{\text {spec }}\left(u_{i}, U_{i-1}\right)=\emptyset$;

(2) Let $R=\left(u_{i}, v, w_{i}\right)$ be a rainbow path with $u_{i} \in U_{i}^{\text {good }}$ and $w_{i} \in U_{i}$. Then $c_{u_{i}}$ appears on $R$. In particular, $c\left(\left\{u_{i}, v\right\}\right)=c_{u_{i}}$ or $(G, c)$ admits a properly colored $\ell$-cycle $C_{\ell}$;

(3) Let $R=\left(u_{i}, v, u_{i-1}\right)$ be a rainbow path with $u_{i} \in U_{i}^{\text {good }}$ and $u_{i-1} \in U_{i-1}^{\text {good }}$. Then $c_{u_{i}}=c_{u_{i-1}}$ or $c_{u_{i}}$ or $c_{u_{i-1}}$ appears on $R$. As well, if $c\left(\left\{u_{i}, v\right\}\right) \neq c_{u_{i}}$ and $c\left(\left\{u_{i-1}, v\right\}\right) \neq c_{u_{i-1}}$, then $(G, c)$ admits a properly colored $\ell$-cycle $C_{\ell}$;

(4) Let $\ell \neq 5$, and let $u_{i}, v_{i} \in U_{i}^{\text {good }}$ and $w_{i}, x_{i} \in U_{i}$ span disjoint edges $\left\{u_{i}, w_{i}\right\},\left\{v_{i}, x_{i}\right\} \in E(G)$. Then $c_{u_{i}}, c_{v_{i}}, c\left(\left\{u_{i}, w_{i}\right\}\right)$, and $c\left(\left\{v_{i}, x_{i}\right\}\right)$ can't all be distinct.

(5) Let $\ell \neq 5$, and let $T_{i} \subseteq U_{i}^{\text {good }}$ be a set with the property that for all $u_{i} \in T_{i}$, there exist $v_{i} \neq w_{i} \in N_{G}\left(u_{i}, U_{i}\right)$ so that $c\left(\left\{u_{i}, v_{i}\right\}\right), c\left(\left\{u_{i}, w_{i}\right\}\right)$, and $c_{u_{i}}$ are all distinct. Then $\left|T_{i}\right| \leq 5$.

Proof of Corollary 7.2, Let $\ell \equiv 2(\bmod 3)$ and fix $i \in \mathbb{Z}_{3}$. For Statement (1), fix $u_{i} \in U_{i}^{\text {good }}$. If $u_{i-1} \in N_{G}^{\text {spec }}\left(u_{i}, U_{i-1}\right)$, then $\left\{u_{i}, u_{i-1}\right\}$ is a $c_{u_{i}}$-free rainbow path which Proposition 7.1 guarantees can be extended to a strong rainbow $\ell$-cycle $C_{\ell}$ (by setting $j=i-1$ and $k=2$, and with $\left.\ell \equiv 2(\bmod 3)\right)$, which contradicts (67).

For Statement $(2)$, let $R=\left(u_{i}, v, w_{i}\right)$ be a rainbow path with $u_{i} \in U_{i}^{\text {good }}$ and $w_{i} \in U_{i}$. If $R$ is $c_{u_{i}}$-free, then Proposition 7.1 guarantees that $R$ can be extended to a strong rainbow $\ell$-cycle $C_{\ell}$ (by setting $j=i$ and $k=3$, and with $\ell \equiv 2(\bmod 3))$, which again contradicts (67). In particular, if $c\left(\left\{u_{i}, v\right\}\right) \neq c_{u_{i}}$, then Proposition 7.1 guarantees that $R$ can be extended to a strong properly colored $\ell$-cycle $C_{\ell}$.

For Statement (3), let $R=\left(u_{i}, v, u_{i-1}\right)$ be a rainbow path with $u_{i} \in U_{i}^{\text {good }}$ and $u_{i-1} \in U_{i-1}^{\text {good }}$. Assume for contradiction that $R$ avoids both $c_{u_{i}} \neq c_{u_{i-1}}$. Since $u_{i-1} \in U_{i-1}^{\text {good }}$ is a good vertex, Proposition 6.2 guarantees a vertex $u_{i-2} \in N_{G}\left(u_{i-1}, U_{i-2}^{\text {good }}\right)$ distinct from $v$ for which $c\left(\left\{u_{i-1}, u_{i-2}\right\}\right)=c_{u_{i-1}}$. Then the path $S=\left(u_{i}, v, u_{i-1}, u_{i-2}\right)$ is rainbow (because $R$ is rainbow and avoids $c_{u_{i-1}}$ ), and the path $S$ avoids $c_{u_{i}}$ (because $R$ does and because $c_{u_{i}} \neq c_{u_{i-1}}$ ). As such, Proposition 7.1 guarantees that $S$ can be extended to a strong rainbow $\ell$-cycle $C_{\ell}$ (by setting $j=i-2$ and $k=4$, and with $\ell \equiv 2$ (mod $3)$ ), which contradicts (67). In particular, assume $c\left(\left\{u_{i}, v\right\}\right) \neq c_{u_{i}}$ and $c\left(\left\{u_{i-1}, v\right\}\right) \neq c_{u_{i-1}}$. Then $S=\left(u_{i}, v, u_{i-1}, u_{i-2}\right)$ is proper (because $R$ is rainbow and $\left.c\left(\left\{u_{i-1}, v\right\}\right) \neq c_{u_{i-1}}\right)$. Since $c\left(\left\{u_{i}, v\right\}\right) \neq c_{u_{i}}$, Proposition 7.1 guarantees that $S$ can be extended to a strong properly colored $\ell$-cycle $C_{\ell}$.

For Statement (4), let $\ell \neq 5$, and let $u_{i}, v_{i} \in U_{i}^{\text {good }}$ and $w_{i}, x_{i} \in U_{i}$ span disjoint edges $\left\{u_{i}, w_{i}\right\},\left\{v_{i}, x_{i}\right\} \in$ $E(G)$. Assume, on the contrary, that $C=\left\{c_{u_{i}}, c_{v_{i}}, c\left(\left\{u_{i}, w_{i}\right\}\right), c\left(\left\{v_{i}, x_{i}\right\}\right)\right\}$ is a set of four distinct colors. Fix any $u_{i+1} \in N_{G}\left(w_{i}, U_{i+1}^{\text {good }}\right)$ where the edge $\left\{w_{i}, u_{i+1}\right\} \in E(G)$ is $C$-free (which is possible by the argument in (82)). Now, fix any

$$
u_{i-1} \in N_{G}\left(u_{i+1}, U_{i-1}\right) \cap N_{G}^{\text {typ }}\left(v_{i}, U_{i-1}\right)
$$

where the edge $\left\{u_{i-1}, u_{i+1}\right\} \in E(G)$ is $\left(C \cup c\left(\left\{w_{i}, u_{i+1}\right\}\right)\right)$-free (which is possible by the argument in (83)). Now, $\left(u_{i}, w_{i}, u_{i+1}, u_{i-1}, v_{i}, x_{i}\right)$ is a rainbow path avoiding $c_{u_{i}}$, which Proposition 7.1 guarantees 
can be extended to a strong rainbow $\ell$-cycle $C_{\ell}$ (by setting $j=i$ and $k=6$, and with $\ell \equiv 2(\bmod 3)$ ), which again contradicts (67).

For Statement (5), let $T_{i} \subseteq U_{i}^{\text {good }}$ be a set with the property so described, but assume for contradiction that $\left|T_{i}\right| \geq 6$. Fix $u_{i} \in T_{i}$, where we take $c_{u_{i}}$ to be blue, and let $v_{i} \neq w_{i} \in N_{G}\left(u_{i}, U_{i}\right)$ be guaranteed by the definition of $T_{i}$, where we take $c\left(\left\{u_{i}, v_{i}\right\}\right)$ to be red and $c\left(\left\{u_{i}, w_{i}\right\}\right)$ to be yellow. Since $\left|T_{i}\right| \geq 6$, there exists $x_{i} \in T_{i} \backslash\left\{u_{i}, v_{i}, w_{i}\right\}$ where $c_{x_{i}}$ is neither red nor yellow. Since $x_{i} \neq u_{i}$, Corollary6.3 guarantees that $c_{x_{i}} \neq c_{u_{i}}$ can't be blue, so we take $c_{x_{i}}$ to be green. Let $y_{i} \neq z_{i} \in N_{G}\left(x_{i}, U_{i}\right)$ be guaranteed by the definition of $T_{i}$. We now distinguish the extent to which $\left\{u_{i}, v_{i}, w_{i}\right\}$ and $\left\{x_{i}, y_{i}, z_{i}\right\}$ overlap.

Case $1\left(\left\{u_{i}, v_{i}, w_{i}\right\} \cap\left\{x_{i}, y_{i}, z_{i}\right\}=\emptyset\right)$. If $c\left(\left\{x_{i}, y_{i}\right\}\right)$ is yellow, then $\left\{u_{i}, v_{i}\right\}$ and $\left\{x_{i}, y_{i}\right\}$ violate Statement (4) above. Similarly, if $c\left(\left\{x_{i}, y_{i}\right\}\right)$ is red, then $\left\{u_{i}, w_{i}\right\}$ and $\left\{x_{i}, y_{i}\right\}$ violate the same. Assume neither $\left\{x_{i}, y_{i}\right\}$ nor $\left\{x_{i}, z_{i}\right\}$ is red or yellow, where the definition of $T_{i}$ ensures neither is green. At most one of these pairs can be blue, so assume $\left\{x_{i}, y_{i}\right\}$ is neither red, yellow, green, nor blue. Now, $\left\{u_{i}, v_{i}\right\}$ and $\left\{x_{i}, y_{i}\right\}$ violate Statement (4) above.

Case $2\left(u_{i} \in\left\{y_{i}, z_{i}\right\}\right)$. Assume w.l.o.g. that $u_{i}=z_{i}$. If $c\left(\left\{u_{i}, x_{i}\right\}\right)$ is yellow, then $\left(x_{i}, u_{i}, v_{i}\right)$ violates Statement (2) above. If $c\left(\left\{u_{i}, x_{i}\right\}\right)$ is not yellow, then it is also not green by the definition of $T_{i}$, and so $\left(x_{i}, u_{i}, w_{i}\right)$ violates the same Statement $(2)$.

Remark. Since $x_{i} \in U_{i}^{\text {good }} \backslash\left\{u_{i}, v_{i}, w_{i}\right\}$, we do not have the case $x_{i} \in\left\{v_{i}, w_{i}\right\}$.

Case $3\left(u_{i} \notin\left\{y_{i}, z_{i}\right\} ;\left\{v_{i}, w_{i}\right\} \cap\left\{y_{i}, z_{i}\right\} \neq \emptyset\right)$. Assume w.l.o.g. that $w_{i}=y_{i}$. If $c\left(\left\{x_{i}, y_{i}\right\}\right)$ is yellow, then $\left\{u_{i}, v_{i}\right\}$ and $\left\{x_{i}, y_{i}\right\}$ violate Statement (4) above. If $c\left(\left\{x_{i}, y_{i}\right\}\right)$ is red, then $\left(u_{i}, w_{i}=y_{i}, x_{i}\right)$ violates Statement (2) above. If $c\left(\left\{x_{i}, y_{i}\right\}\right)$ is blue, then $\left(x_{i}, y_{i}=w_{i}, u_{i}\right)$ violates Statement (2) above. Otherwise, $c\left(\left\{x_{i}, y_{i}\right\}\right)$ isn't green by the definition of $T_{i}$, so $\left\{u_{i}, v_{i}\right\}$ and $\left\{x_{i}, y_{i}\right\}$ violate Statement (4) above.

7.2. Proof of Lemma 2.9; Statement $(1)$ when $\ell \equiv \mathbf{2}(\bmod 3)$. Let $\ell \equiv 2(\bmod 3)$, where $\ell \neq 5$. The hypothesis of Statement (1) of Lemma 2.9 gives that $\delta^{c}(G) \geq(n+5) / 3$. Assume w.l.o.g. that

$$
\left|U_{2}\right| \leq\left|U_{1}\right| \leq\left|U_{0}\right|, \quad \text { in which case } \quad\left|U_{2}\right| \leq\left\lfloor\frac{n}{3}\right\rfloor \leq\left\lceil\frac{n}{3}\right\rceil \leq\left|U_{0}\right| .
$$

In the immediate sequel, we motivate the main approach of the proof.

7.2.1. Main idea of proof. We shall make repeated use of Statement (5) of Corollary 7.2, for which we establish the following notation. Fix $i \in \mathbb{Z}_{3}$ and $u_{i} \in U_{i}^{\text {good }}$, and define

$$
c^{\mathrm{spec}}\left(u_{i}, U_{i}\right)=\left\{c\left(\left\{u_{i}, v_{i}\right\}\right) \neq c_{u_{i}}: v_{i} \in N_{G}\left(u_{i}, U_{i}\right)\right\}
$$

for the set of special (non-primary) colors on edges $\left\{u_{i}, v_{i}\right\} \in E_{G}\left(u_{i}, U_{i}\right)$ incident to $u_{i}$ in $U_{i}$. By Statement (1) of Corollary 7.2. all edges $\left\{u_{i}, u_{i-1}\right\} \in E_{G}\left(u_{i}, U_{i-1}\right)$ are colored by $c_{u_{i}}$, and so

$$
\begin{gathered}
\left|c^{\mathrm{spec}}\left(u_{i}, U_{i}\right)\right| \geq \operatorname{deg}_{G}^{c}\left(u_{i}\right)-\operatorname{deg}_{G}^{c}\left(u_{i}, U_{i-1}\right)-\operatorname{deg}_{G}^{c}\left(u_{i}, U_{i+1}\right)=\operatorname{deg}_{G}^{c}\left(u_{i}\right)-1-\operatorname{deg}_{G}^{c}\left(u_{i}, U_{i+1}\right) \\
\geq \delta^{c}(G)-1-\left|U_{i+1}\right| \geq \frac{n+5}{3}-1-\left|U_{i+1}\right|=\frac{n+2}{3}-\left|U_{i+1}\right| .
\end{gathered}
$$

In particular, when $i=1 \in \mathbb{Z}_{3}$, we infer that every $u_{1} \in U_{1}^{\text {good }}$ satisfies

$$
\left|c^{\mathrm{spec}}\left(u_{1}, U_{1}\right)\right| \geq \frac{n+2}{3}-\left|U_{2}\right| \stackrel{\frac{84}{\geq}}{\geq} \frac{n+2}{3}-\left\lfloor\frac{n}{3}\right\rfloor .
$$

As such, if $n \equiv 2(\bmod 3)$, then (87) gives $\left|c^{\text {spec }}\left(u_{1}, U_{1}\right)\right| \geq 2$ for every $u_{1} \in U_{1}^{\text {good }}$, and so $T_{1}=$ $U_{1}^{\text {good }}$ readily contradicts Statement (5) of Corollary 7.2 (because $\left|U_{1}^{\text {good }}\right|$ from (75) is much too large). Similarly, if $\left|U_{2}\right| \leq\lfloor n / 3\rfloor-1$, then (87) gives $\left|c^{\text {spec }}\left(u_{1}, U_{1}\right)\right| \geq 2$ for every $u_{1} \in U_{1}^{\text {good }}$, giving the same contradiction. The main idea of the current proof exploits a similar theme to the instances $n \equiv 2$ (mod 
3) or $\left|U_{2}\right| \leq\lfloor n / 3\rfloor-1$, which we announce as our goal:

we seek to determine a large set $T_{i} \subseteq U_{i}^{\text {good }}$, for some $i \in \mathbb{Z}_{3}$,

$$
\text { where every } u_{i} \in T_{i} \text { satisfies }\left|c^{\mathrm{spec}}\left(u_{i}, U_{i}\right)\right| \geq 2 \text {. }
$$

When so, we contradict Statement (5) of Corollary 7.2

7.2.2. Supporting details. From the discussion above, it suffices to consider the case $n \not \equiv 2(\bmod 3)$ and $\left|U_{2}\right|=\lfloor n / 3\rfloor$. As such, $\left|U_{2}\right|=\left|U_{1}\right|=\lfloor n / 3\rfloor$ and $\left|U_{0}\right|=\lceil n / 3\rceil$. Now, for $u_{1} \in U_{1}^{\text {good }}$, we define

$$
S\left(u_{1}\right)=\left\{v_{1} \in N_{G}\left(u_{1}, U_{1}\right): c\left(\left\{u_{1}, v_{1}\right\}\right) \neq c_{u_{1}}\right\} .
$$

We refine the partition $U_{1}=U_{1}^{\text {good }} \cup U_{1}^{\text {bad }}$ from (75) by subdividing $U_{1}^{\text {good }}$ into

$$
A_{1}=\left\{u_{1} \in U_{1}^{\text {good }}: S\left(u_{1}\right) \cap U_{1}^{\text {bad }} \neq \emptyset\right\} \quad \text { and } \quad B_{1}=U_{1}^{\text {good }} \backslash A_{1} .
$$

We will observe the following fact.

Fact 7.3. Every $u_{1} \in B_{1}$ satisfies $S\left(u_{1}\right) \subseteq A_{1}$.

Proof of Fact 7.3. Fix $u_{1} \in B_{1}$, but assume for contradiction that $v_{1} \in S\left(u_{1}\right) \cap B_{1}$. Since both $u_{1} \neq v_{1} \in U_{1}^{\text {good }}$ are good vertices, Corollary 6.3 guarantees that $c_{u_{1}} \neq c_{v_{1}}$, where we will take $c_{u_{1}}$ to be red and $c_{v_{1}}$ to be blue. From $v_{1} \in S\left(u_{1}\right)$, we infer that $c\left(\left\{u_{1}, v_{1}\right\}\right)$ is not $c_{u_{1}}=$ red. We distinguish two cases.

Case $1\left(c\left(\left\{u_{1}, v_{1}\right\}\right) \neq c_{v_{1}}\right)$. Here, we will take $c\left(\left\{u_{1}, v_{1}\right\}\right)$ to be yellow. Proposition 6.2 guarantees a vertex $u_{0} \in N_{G}^{\text {typ }}\left(v_{1}, U_{0}^{\text {good }}\right)$ so that $c\left(\left\{u_{0}, v_{1}\right\}\right)=c_{v_{1}}$ is blue but $c_{u_{0}}$ is neither red, blue, nor yellow. We take $c_{u_{0}}$ to be green. Now, $R=\left(u_{1}, v_{1}, u_{0}\right)$ is a rainbow path where $u_{1} \in U_{1}^{\text {good }}$, where $u_{0} \in U_{0}^{\text {good }}$, but where neither $c_{u_{1}} \neq c_{u_{0}}$ (red nor green) appear on $R$, which contradicts Statement (3) of Corollary 7.2

Case $2\left(c\left(\left\{u_{1}, v_{1}\right\}\right)=c_{v_{1}}\right)$. From (87), we infer that $\left|S\left(v_{1}\right)\right| \geq 1$, where $u_{1} \notin S\left(v_{1}\right)$ on account that $c\left(\left\{u_{1}, v_{1}\right\}\right)=c_{v_{1}}$ is blue. From $v_{1} \in B_{1}$, we infer that $S\left(v_{1}\right) \cap U_{1}^{\text {bad }}=\emptyset$, and so there exists $u_{1} \neq w_{1} \in S\left(v_{1}\right) \subseteq U_{1}^{\text {good }}$. From $w_{1} \in S\left(v_{1}\right)$, we infer that $c\left(\left\{v_{1}, w_{1}\right\}\right) \neq c_{v_{1}}$ is not blue. So the path $\left(u_{1}, v_{1}, w_{1}\right)$ is rainbow, and Statement (2) of Corollary 7.2 implies that $c\left(\left\{v_{1}, w_{1}\right\}\right)$ is $c_{u_{1}}$ (red). Since $u_{1}, v_{1}, w_{1} \in U_{1}^{\text {good }}$ are good and distinct, Corollary 6.3 guarantees that the primary colors $c_{u_{1}}(\mathrm{red}), c_{v_{1}}$ (blue), and $c_{w_{1}}$ are distinct, where we take $c_{w_{1}}$ to be green. Since $w_{1} \in U_{1}^{\text {good }}$ is good, Proposition 6.2 guarantees a vertex $u_{0} \in N_{G}^{\text {typ }}\left(w_{1}, U_{0}^{\text {good }}\right)$ so that $c_{u_{0}}$ is neither $c_{v_{1}}$ (blue), $c\left(\left\{u_{0}, w_{1}\right\}\right)$ (green), nor $c\left(\left\{v_{1}, w_{1}\right\}\right)$ (red). Now, $\left(v_{1}, w_{1}, u_{0}\right)$ contradicts Statement (3) of Corollary 7.2,

Fact 7.3 admits the following corollary.

Corollary 7.4. There exist distinct $u_{1}, v_{1}, w_{1} \in U_{1}^{\text {good }}$ satisfying $S\left(u_{1}\right) \cap S\left(v_{1}\right) \cap S\left(w_{1}\right) \neq \emptyset$.

Proof of Corollary 7.4. Define the auxiliary directed graph $\vec{\Gamma}=\left(U_{1}, \vec{E}\right)$ by the rule that for each $\left(u_{1}, v_{1}\right) \in U_{1} \times U_{1}$, we put $\left(u_{1}, v_{1}\right) \in \vec{E}$ if, and only if, $v_{1} \in S\left(u_{1}\right)$. In this notation, $S\left(u_{1}\right)=N_{\vec{\Gamma}}^{+}\left(u_{1}\right)$. We now distinguish two cases.

Case $1\left(\left|B_{1}\right|>2\left|A_{1}\right|\right)$. For the bipartition $A_{1} \cup B_{1}$ (cf. (89)), we infer

$$
\sum_{a_{1} \in A_{1}}\left|N_{\vec{\Gamma}}^{-}\left(a_{1}\right) \cap B_{1}\right|=\sum_{b_{1} \in B_{1}}\left|N_{\vec{\Gamma}}^{+}\left(b_{1}\right) \cap A_{1}\right|=\sum_{b_{1} \in B_{1}}\left|S\left(b_{1}\right) \cap A_{1}\right| \stackrel{\text { Fct } \stackrel{7.3}{=}}{=} \sum_{b_{1} \in B_{1}}\left|S\left(b_{1}\right)\right| \stackrel{\sqrt[87]{\geq}}{\geq}\left|B_{1}\right|>2\left|A_{1}\right| .
$$

By averaging, there exists $\bar{a}_{1} \in A_{1}$ which satisfies $\left|N_{\vec{\Gamma}}^{-}\left(\bar{a}_{1}\right) \cap B_{1}\right| \geq 3$, so let $b_{1}, b_{1}^{\prime}, b_{1}^{\prime \prime} \in N_{\vec{\Gamma}}^{-}\left(\bar{a}_{1}\right)$. Then

$$
\bar{a}_{1} \in N_{\Gamma}^{+}\left(b_{1}\right) \cap N_{\Gamma}^{+}\left(b_{1}^{\prime}\right) \cap N_{\Gamma}^{+}\left(b_{1}^{\prime \prime}\right)=S\left(b_{1}\right) \cap S\left(b_{1}^{\prime}\right) \cap S\left(b_{1}^{\prime \prime}\right),
$$

and so $S\left(b_{1}\right) \cap S\left(b_{1}^{\prime}\right) \cap S\left(b_{1}^{\prime \prime}\right) \neq \emptyset$. 
Case $2\left(\left|B_{1}\right| \leq 2\left|A_{1}\right|\right)$. For the bipartition $A_{1} \cup U_{1}^{\text {bad }}\left(\right.$ recall $\left.A_{1} \subseteq U_{1}^{\text {good }}\right)$, we infer

$$
\sum_{u_{1} \in U_{1}^{\text {bad }}}\left|N_{\vec{\Gamma}}^{-}\left(u_{1}\right) \cap A_{1}\right|=\sum_{a_{1} \in A_{1}}\left|N_{\vec{\Gamma}}^{+}\left(a_{1}\right) \cap U_{1}^{\text {bad }}\right|=\sum_{a_{1} \in A_{1}}\left|S\left(a_{1}\right) \cap U_{1}^{\text {bad }}\right| \stackrel{\text { def }}{\geq}\left|A_{1}\right|,
$$

where we used the definition of $A_{1}$ from (89). Moreover, from the bipartition $U_{1}^{\text {good }}=A_{1} \cup B_{1}$, we infer

$$
\begin{aligned}
& 3\left|A_{1}\right| \geq\left|A_{1}\right|+\left|B_{1}\right|=\left|U_{1}^{\text {good }}\right| \stackrel{\text { 75 }}{\geq}\left(\frac{1}{3}-75 \lambda^{1 / 4}\right) n \\
& \stackrel{65}{\geq} 648 \lambda^{1 / 4} n \stackrel{\text { 75 }}{\geq} 9\left|U_{1}^{\mathrm{bad}}\right| \quad \Longrightarrow \quad\left|A_{1}\right| \geq 3\left|U_{1}^{\mathrm{bad}}\right| .
\end{aligned}
$$

Combining (90) and (91) yields $\sum_{u_{1} \in U_{1}^{\text {bad }}}\left|N_{\vec{\Gamma}}^{-}\left(u_{1}\right) \cap A_{1}\right| \geq 3\left|U_{1}^{\text {bad }}\right|$, and so an average vertex $\bar{u}_{1} \in U_{1}^{\text {bad }}$ satisfies $\left|N_{\vec{\Gamma}}^{-}\left(\bar{u}_{1}\right) \cap A_{1}\right| \geq 3$. Let $a_{1}, a_{1}^{\prime}, a_{1}^{\prime \prime} \in N_{\vec{\Gamma}}^{-}\left(\bar{u}_{1}\right)$, in which case

$$
\bar{u}_{1} \in N_{\Gamma}^{+}\left(a_{1}\right) \cap N_{\Gamma}^{+}\left(a_{1}^{\prime}\right) \cap N_{\Gamma}^{+}\left(a_{1}^{\prime \prime}\right)=S\left(a_{1}\right) \cap S\left(a_{1}^{\prime}\right) \cap S\left(a_{1}^{\prime \prime}\right),
$$

and so $S\left(a_{1}\right) \cap S\left(a_{1}^{\prime}\right) \cap S\left(a_{1}^{\prime \prime}\right) \neq \emptyset$.

For the remainder of the proof, we fix distinct $u_{1}, v_{1}, w_{1} \in U_{1}^{\text {good }}$ guaranteed by Corollary 7.4. We also fix an element $x_{1} \in S\left(u_{1}\right) \cap S\left(v_{1}\right) \cap S\left(w_{1}\right)$. We garner the following useful corollary.

Corollary 7.5. The coloring $c$ is constant on the edges $E_{G}\left(x_{1}, U_{1}\right)$.

Proof of Corollary 7.5. We first show that

$$
c\left(\left\{u_{1}, x_{1}\right\}\right)=c\left(\left\{v_{1}, x_{1}\right\}\right)=c\left(\left\{w_{1}, x_{1}\right\}\right) .
$$

For that, since $u_{1}, v_{1}, w_{1} \in U_{1}^{\text {good }}$ are distinct good vertices, Corollary 6.3 guarantees that $c_{u_{1}}, c_{v_{1}}$, and $c_{w_{1}}$ are distinct, so we take $c_{u_{1}}$ to be red, $c_{v_{1}}$ to be blue, and $c_{w_{1}}$ to be yellow. Assume, on the contrary, that $c\left(\left\{u_{1}, x_{1}\right\}\right) \neq c\left(\left\{v_{1}, x_{1}\right\}\right)$. Then $\left(u_{1}, x_{1}, v_{1}\right)$ is a rainbow $U_{1}$-path where $u_{1} \in U_{1}^{\text {good }}$ is a good vertex, so Statement (2) of Corollary 7.2 guarantees that $c\left(\left\{x_{1}, v_{1}\right\}\right)$ is $c_{u_{1}}=$ red. Applying the same argument to $\left(v_{1}, x_{1}, u_{1}\right)$, we infer that $c\left(\left\{u_{1}, x_{1}\right\}\right)$ is $c_{v_{1}}=$ blue. Now, $c_{w_{1}}=$ yellow appears on neither $\left(w_{1}, x_{1}, u_{1}\right)$ nor $\left(w_{1}, x_{1}, v_{1}\right)$ (since $x_{1} \in S\left(w_{1}\right)$ guarantees that $c\left(\left\{w_{1}, x_{1}\right\}\right)$ is not $c_{w_{1}}=$ yellow). Since $w_{1} \in U_{1}^{\text {good }}$ is a good vertex, Statement (2) of Corollary 7.2 guarantees that both $\left(w_{1}, x_{1}, u_{1}\right)$ and $\left(w_{1}, x_{1}, v_{1}\right)$ are monochromatic, and so $c\left(\left\{w_{1}, x_{1}\right\}\right)$ is both red and blue, a contradiction.

Corollary 7.5 now easily follows from (92), where we take that common color to be green. By the argument above, any edge $\left\{x_{1}, y_{1}\right\} \in E_{G}\left(x_{1}, U_{1}\right)$ that isn't colored green must be colored each of red, blue, and yellow, which isn't possible.

7.2.3. Finale. We return to our goal in (88). Let $u_{1}, v_{1}, w_{1} \in U_{1}^{\text {good }}$ and $x_{1} \in S\left(u_{1}\right) \cap S\left(v_{1}\right) \cap S\left(w_{1}\right)$ be fixed from the previous subsection, where all of $E_{G}\left(x_{1}, U_{1}\right)$ is colored green, which is the only color from before which we now need to reference. Then $E_{G}\left(x_{1}, U_{1} \cup U_{2}\right)$ admits at most $\left|U_{2}\right|+1$ colors, the set of which we call $C=C\left(x_{1}, U_{1}, U_{2}\right)$. As such, the number of non- $C$ colors on $E_{G}\left(x_{1}, U_{0}\right)$ is at least

$\operatorname{deg}_{G}^{c}\left(x_{1}\right)-\operatorname{deg}_{G}^{c}\left(x_{1}, U_{1}\right)-\operatorname{deg}_{G}^{c}\left(x_{1}, U_{2}\right) \geq \delta^{c}(G)-1-\left|U_{2}\right| \geq \frac{n+5}{3}-1-\left|U_{2}\right|=\frac{n+2}{3}-\left|U_{2}\right| \stackrel{\frac{84}{\geq}}{\geq} \frac{n+2}{3}-\left\lfloor\frac{n}{3}\right\rfloor$, which is positive. Fix $u_{0} \in N_{G}\left(x_{1}, U_{0}\right)$ where $c\left(\left\{u_{0}, x_{1}\right\}\right) \notin C$. In particular, $c\left(\left\{u_{0}, x_{1}\right\}\right)$ is not green, and we take $c\left(\left\{u_{0}, x_{1}\right\}\right)$ to be purple. (It won't matter if $c\left(\left\{u_{0}, x_{1}\right\}\right)$ appeared in the previous subsection, so long as $c\left(\left\{u_{0}, x_{1}\right\}\right)$ is not green.) Define

$$
T_{0}=\left\{v_{0} \in U_{0}^{\text {good }}: v_{0} \neq u_{0}, c_{v_{0}} \neq c\left(\left\{u_{0}, x_{1}\right\}\right)=\text { purple, } c_{v_{0}} \neq \text { green }\right\},
$$

where Corollary 6.3 guarantees

$$
\left|T_{0}\right| \geq\left|U_{0}^{\text {good }}\right|-3 \stackrel{\frac{75}{\geq}}{\geq}\left(\frac{1}{3}-75 \lambda^{1 / 4}\right) n-3=\Omega(n) .
$$

We make the following critical observation. 
Observation 7.6. An edge $\left\{v_{0}, x_{1}\right\} \in E_{G}\left(x_{1}, T_{0}\right)$ must be colored $c_{v_{0}}$.

Proof of Observation 7.6. For a fixed $\left\{v_{0}, x_{1}\right\} \in E_{G}\left(x_{1}, T_{0}\right)$, we distinguish two cases.

Case $1\left(c\left(\left\{v_{0}, x_{1}\right\}\right) \neq c\left(\left\{u_{0}, x_{1}\right\}\right)=\right.$ purple $)$. Here, $\left(v_{0}, x_{1}, u_{0}\right)$ is a rainbow path where $v_{0} \in T_{0} \subseteq U_{0}^{\text {good }}$ is a good vertex. Statement (2) of Corollary 7.2 guarantees that $c_{v_{0}}$ appears on $\left(v_{0}, x_{1}, u_{0}\right)$, and since $c_{v_{0}} \neq c\left(\left\{u_{0}, x_{1}\right\}\right)=$ purple holds by the definition of $T_{0}$, we must have $c\left(\left\{v_{0}, x_{1}\right\}\right)=c_{v_{0}}$.

Case $2\left(c\left(\left\{v_{0}, x_{1}\right\}\right)=c\left(\left\{u_{0}, x_{1}\right\}\right)=\right.$ purple $)$. Among the fixed distinct vertices $u_{1}, v_{1}, w_{1} \in U_{1}^{\text {good }}$ above, Corollary 6.3 guarantees that at most one of the distinct colors $c_{u_{1}}, c_{v_{1}}, c_{w_{1}}$ can equal $c\left(\left\{u_{0}, x_{1}\right\}\right)=$ $c\left(\left\{v_{0}, x_{1}\right\}\right)=$ purple, and at most one of $c_{u_{1}}, c_{v_{1}}, c_{w_{1}}$ can equal $c_{v_{0}}$. Assume w.l.o.g. that

$$
c_{v_{0}} \neq c_{u_{1}} \neq c\left(\left\{u_{0}, x_{1}\right\}\right)=c\left(\left\{v_{0}, x_{1}\right\}\right)=\text { purple. }
$$

Now, the path $\left(u_{1}, x_{1}, v_{0}\right)$ is a green-purple rainbow path where $u_{1} \in U_{1}^{\text {good }}$ and $v_{0} \in T_{0} \subseteq U_{0}^{\text {good }}$ are good vertices satisfying $c_{u_{1}} \neq c_{v_{0}}$. Statement (3) guarantees that one of $c_{v_{0}} \neq c_{u_{1}}$ appears on $\left(u_{1}, x_{1}, v_{0}\right)$, but neither do. Indeed, $c_{v_{0}}$ is neither green nor purple by the definition of $T_{0}$ (cf. (93)), and $c_{u_{1}}$ is not green by $x_{1} \in S\left(u_{1}\right)$ and it is not purple by our choice above.

We now conclude the proof of Statement $(1)$ of Lemma 2.9 when $\ell \equiv 2(\bmod 3)$. Fix a vertex $v_{0} \in T_{0}$. By combining Statement (1) of Corollary 7.2 with Observation 7.6, we conclude that all edges $E_{G}\left(v_{0}, U_{2} \cup\left\{x_{1}\right\}\right)$ are colored the single primary color $c_{v_{0}}$. However distinctly the edges $E_{G}\left(v_{0}, U_{1} \backslash\left\{x_{1}\right\}\right)$ are colored, the edges $E_{G}\left(v_{0}, U_{1} \cup U_{2}\right)$ are colored with at most $1+\left(\left|U_{1}\right|-1\right)=\left|U_{1}\right|=\lfloor n / 3\rfloor$ many colors, one of which is the primary color $c_{v_{0}}$. (Recall that it suffices to consider the case $\left|U_{2}\right|=\left|U_{1}\right|=\lfloor n / 3\rfloor$.) All remaining colors incident to $v_{0}$ are special and are applied to $E_{G}\left(v_{0}, U_{0}\right)$, the number of which is precisely given by the parameter $\left|c^{\mathrm{spec}}\left(v_{0}, U_{0}\right)\right|$ from (85). Altogether, we conclude

$$
\left|c^{\text {spec }}\left(v_{0}, U_{0}\right)\right| \geq \operatorname{deg}_{G}^{c}\left(v_{0}, U_{0}\right)-\mid c\left(E_{G}\left(v_{0}, U_{1} \cup U_{2}\right) \mid \geq \delta^{c}(G)-\left\lfloor\frac{n}{3}\right\rfloor \geq \frac{n+5}{3}-\left\lfloor\frac{n}{3}\right\rfloor \geq \frac{5}{3},\right.
$$

and therefore $\left|c^{\mathrm{spec}}\left(v_{0}, U_{0}\right)\right| \geq 2$. Now, (94) and (95) together contradict Statement (5) of Corollary 7.2 .

7.3. Proof of Lemma 2.9; Statement $(2)$ when $\ell \equiv \mathbf{2}(\bmod 3)$. Let $\ell \equiv 2(\bmod 3)$. The hypothesis of Statement (2) of Lemma 2.9 gives that $\delta^{c}(G) \geq(n+4) / 3$ (cf. (130) $)$. We again assume w.l.o.g. that (84) holds, and we want to conclude that $(G, c)$ admits a properly colored $\ell$-cycle $C_{\ell}$.

We assume, on the contrary, that $(G, c)$ does not admit a properly colored $\ell$-cycle $C_{\ell}$.

Our assumption in (96) will guarantee vertices $x_{1} \in U_{1}^{\text {good }}$ and $y_{1}, z_{1} \in U_{1}$, where $\left(x_{1}, y_{1}, z_{1}\right)$ is a rainbow $U_{1}$-path satisfying $c\left(\left\{x_{1}, y_{1}\right\}\right) \neq c_{x_{1}}$. Then (96) contradicts Statement (2) of Corollary [7.2.

We begin our work with an observation. Fix an auxiliary vertex $u_{0} \in U_{0}^{\text {good }}$, where Statement (1) of Corollary 7.2 guarantees that $E_{G}\left(u_{0}, U_{2}\right)$ is colored only with $c_{u_{0}}$. We observe that

$$
E_{G}\left(u_{0}, U_{0}\right) \text { is also colored only with } c_{u_{0}} .
$$

To see (97), suppose $v_{0} \in N_{G}\left(u_{0}, U_{0}\right)$ admits $c\left(\left\{u_{0}, v_{0}\right\}\right) \neq c_{u_{0}}$. Let $u_{1} \in N_{G}\left(v_{0}, U_{1}^{\text {good }}\right)$ have color $c\left(\left\{v_{0}, u_{1}\right\}\right) \neq c\left(\left\{u_{0}, v_{0}\right\}\right)$, where we used $\operatorname{deg}_{G}^{c}\left(v_{0}, U_{1}^{\text {good }}\right) \geq\left((1 / 9)-144 \lambda^{1 / 4}\right) n$ implicit in (75)). Statement (1) of Corollary 7.2 guarantees that $c\left(\left\{v_{0}, u_{1}\right\}\right)=c_{u_{1}}$. As such, the number $\left|c^{\mathrm{spec}}\left(u_{1}, U_{1}\right)\right|$ of special colors incident to $u_{1}$ in $U_{1}$ satisfies

$$
\begin{aligned}
&\left|c^{\mathrm{spec}}\left(u_{1}, U_{1}\right)\right| \geq \operatorname{deg}_{G}^{c}\left(u_{1}\right)- \operatorname{deg}_{G}^{c}\left(u_{1}, U_{0}\right)-\operatorname{deg}_{G}^{c}\left(u_{1}, U_{2}\right) \geq \delta^{c}(G)-\operatorname{deg}_{G}^{c}\left(u_{1}, U_{0}\right)-\operatorname{deg}_{G}^{c}\left(u_{1}, U_{2}\right) \\
&=\delta^{c}(G)-1-\operatorname{deg}_{G}^{c}\left(u_{1}, U_{2}\right) \geq \frac{n+4}{3}-1-\left|U_{2}\right| \frac{84}{\geq} \frac{n+1}{3}-\left\lfloor\frac{n}{3}\right\rfloor \geq \frac{1}{3},
\end{aligned}
$$

so fix $v_{1} \in N_{G}\left(u_{1}, U_{1}\right)$ where $c\left(\left\{u_{1}, v_{1}\right\}\right) \neq c_{u_{1}}$. Now, $P=\left(u_{0}, v_{0}, u_{1}, v_{1}\right)$ is a properly colored path where $c\left(\left\{u_{0}, v_{0}\right\}\right) \neq c_{u_{0}}$. Proposition 7.1 guarantees (with $i=0, j=1, k=4$, and $\ell \equiv 2(\bmod 3)$ ) that $P$ can be extended to a strong rainbow $\ell$-cycle $C_{\ell}$, contradicting (96). This proves (97). 
We choose the first promised vertex $x_{1} \in U_{1}^{\text {good }}$ arbitrarily, where the auxiliary vertex $u_{0} \in U_{0}^{\text {good }}$ above is still fixed. To choose the second promised vertex $y_{1} \in U_{1}$, define

$$
A_{u_{0}}=\left\{u_{1} \in N_{G}\left(u_{0}, U_{1}\right): c\left(\left\{u_{0}, u_{1}\right\}\right) \neq c_{u_{0}}\right\} \text { and } B_{x_{1}}=\left\{v_{1} \in N_{G}\left(x_{1}, U_{1}\right): c\left(\left\{x_{1}, v_{1}\right\}\right) \neq c_{x_{1}}\right\} .
$$

(The set $B_{x_{1}}$ is the same as $S\left(x_{1}\right)$ from the previous subsection.) Then $A_{u_{0}} \cup B_{x_{1}} \subseteq U_{1}$, and so

$$
\left|A_{u_{0}} \cap B_{x_{1}}\right|=\left|A_{u_{0}}\right|+\left|B_{x_{1}}\right|-\left|A_{u_{0}} \cup B_{x_{1}}\right| \geq\left|A_{u_{0}}\right|+\left|B_{x_{1}}\right|-\left|U_{1}\right| .
$$

From our observation above (cf. (97)), all of $E_{G}\left(u_{0}, U_{0} \cup U_{2}\right)$ is colored with $c_{u_{0}}$, and therefore $\left|A_{u_{0}}\right| \geq$ $\operatorname{deg}_{G}^{c}\left(u_{0}\right)-1$. Since $x_{1} \in U_{1}^{\text {good }}$ is a good vertex, Statement (1) of Corollary 7.2 guarantees that all of $E_{G}\left(x_{1}, U_{0}\right)$ is colored with $c_{x_{1}}$, and therefore

$$
\left|B_{x_{1}}\right| \geq \operatorname{deg}_{G}^{c}\left(x_{1}\right)-\operatorname{deg}_{G}^{c}\left(x_{1}, U_{0}\right)-\operatorname{deg}_{G}^{c}\left(x_{1}, U_{2}\right) \geq \operatorname{deg}_{G}^{c}\left(x_{1}\right)-1-\left|U_{2}\right| .
$$

Returning to (100), we conclude

$$
\begin{aligned}
\left|A_{u_{0}} \cap B_{x_{1}}\right| \geq\left|A_{u_{0}}\right|+\left|B_{x_{1}}\right|-\left|U_{1}\right| \geq \operatorname{deg}_{G}^{c}\left(u_{0}\right)+\operatorname{deg}_{G}^{c}\left(x_{1}\right)-2-\left|U_{1}\right|-\left|U_{2}\right| \\
=\operatorname{deg}_{G}^{c}\left(u_{0}\right)+\operatorname{deg}_{G}^{c}\left(x_{1}\right)-2-\left(n-\left|U_{0}\right|\right) \geq 2 \delta^{c}(G)-2-n+\left|U_{0}\right| \\
\geq 2\left(\frac{n+4}{3}\right)-2-n+\left|U_{0}\right|=\frac{2 n+2}{3}-n+\left|U_{0}\right| \stackrel{\mid 84}{\geq} \frac{2 n+2}{3}-n+\left\lceil\frac{n}{3}\right\rceil \geq \frac{2}{3} .
\end{aligned}
$$

Fix $y_{1} \in A_{u_{0}} \cap B_{x_{1}}$ arbitrarily.

To choose the third promised vertex $z_{1} \in U_{1}$, we make a couple observations. First, we observe that the path $\left(x_{1}, y_{1}, u_{0}\right)$ must be monochromatic. Indeed, since $y_{1} \in A_{u_{0}} \cap B_{x_{1}}$, we infer from (99) that $c\left(\left\{u_{0}, y_{1}\right\}\right) \neq c_{u_{0}}$ and $c\left(\left\{x_{1}, y_{1}\right\}\right) \neq c_{x_{1}}$. Thus, if $\left(x_{1}, y_{1}, u_{0}\right)$ were rainbow, then Statement (3) of Corollary 7.2 would guarantee that $(G, c)$ admits a properly colored $\ell$-cycle $C_{\ell}$, contradicting (96). Henceforth, we take $c\left(\left\{u_{0}, y_{1}\right\}\right)=c\left(\left\{x_{1}, y_{1}\right\}\right)$ to be blue. Second, we observe that

$$
\text { all of } E_{G}\left(y_{1}, U_{0}\right) \text { is colored by } c\left(\left\{u_{0}, y_{1}\right\}\right)=c\left(\left\{x_{1}, y_{1}\right\}\right)=\text { blue. }
$$

Indeed, suppose $\left\{v_{0}, y_{1}\right\} \in E_{G}\left(y_{1}, U_{0}\right)$ admitted $c\left(\left\{v_{0}, y_{1}\right\}\right) \neq c\left(\left\{u_{0}, y_{1}\right\}\right)=$ blue. Then $\left(u_{0}, y_{1}, v_{0}\right)$ is a rainbow path with $c\left(\left\{u_{0}, y_{1}\right\}\right) \neq c_{u_{0}}$ (because $y_{1} \in A_{u_{0}}$ from (99)). Statement (2) of Corollary 7.2 then guarantees that $(G, c)$ admits a properly colored $\ell$-cycle $C_{\ell}$, again contradicting (96). Now, all of $E_{G}\left(y_{1}, U_{0} \cup\left\{x_{1}\right\}\right)$ is colored blue, and so the number of non-blue colors of $E_{G}\left(y_{1}, U_{1}\right)$ is at least

$$
\begin{aligned}
\operatorname{deg}_{G}^{c}\left(y_{1}\right)-\operatorname{deg}_{G}^{c}\left(y_{1}, U_{0}\right)-\operatorname{deg}_{G}^{c}\left(y_{1}, U_{2}\right) & =\operatorname{deg}_{G}^{c}\left(y_{1}\right)-1-\operatorname{deg}_{G}^{c}\left(y_{1}, U_{2}\right) \\
& \geq \delta^{c}(G)-1-\left|U_{2}\right| \geq \frac{n+4}{3}-1-\left|U_{2}\right| \stackrel{\frac{84}{\geq}}{\geq} \frac{n+1}{3}-\left\lfloor\frac{n}{3}\right\rfloor \geq \frac{1}{3} .
\end{aligned}
$$

Fix any $z_{1} \in N_{G}\left(y_{1}, U_{1}\right)$ for which $c\left(\left\{y_{1}, z_{1}\right\}\right)$ is not blue. Since $c\left(\left\{x_{1}, y_{1}\right\}\right)$ is blue, we infer that $\left(x_{1}, y_{1}, z_{1}\right)$ is a rainbow path where $c_{x_{1}} \neq c\left(\left\{x_{1}, y_{1}\right\}\right)=$ blue is guaranteed by $y_{1} \in B_{x_{1}}$ from (99). Thus, Statement (2) of Corollary 7.2 guarantees from the rainbow $U_{1}$-path $\left(x_{1}, y_{1}, z_{1}\right)$ (where $c_{x_{1}} \neq$ $c\left(\left\{x_{1}, y_{1}\right\}\right)=$ blue) that $(G, c)$ admits a properly colored $\ell$-cycle $C_{\ell}$, again contradicting (96).

\section{Proof of Lemma 2.9 - Part 3: Strong or Short Cycles and the Case $\ell \equiv 1$ (mod 3)}

Continuing from the previous sections, we prove Lemma 2.9 in the case $\ell \equiv 1(\bmod 3)$. For this, we will need a number of supporting details, where we begin by establishing an analogue of Corollary 7.2 for $\ell \equiv 1(\bmod 3)$ (another corollary of Proposition 7.1). We use the following terminology and notation. For a fixed $j \in \mathbb{Z}_{3}$, recall the set $U_{j}=U_{j}^{\text {good }} \cup U_{j}^{\text {bad }}$ (cf. (75)). We shall say that a vertex $u_{j} \in U_{j}^{\text {bad }}$ is an internal (bad) vertex if $\operatorname{deg}_{G}^{c}\left(u_{j}, U_{j}\right) \geq 3$, and that $u_{j} \in U_{j}^{\text {bad }}$ is an external (bad) vertex otherwise. We then define

$$
I_{j}^{\mathrm{bad}}=\left\{u_{j} \in U_{j}^{\mathrm{bad}}: \operatorname{deg}_{G}^{c}\left(u_{j}, U_{j}\right) \geq 3\right\} \quad \text { and } \quad E_{j}^{\mathrm{bad}}=\left\{u_{j} \in U_{j}^{\mathrm{bad}}: \operatorname{deg}_{G}^{c}\left(u_{j}, U_{j}\right) \leq 2\right\} .
$$

Corollary 8.1. Let $\ell \equiv 1(\bmod 3)$. Fix an index $j \in \mathbb{Z}_{3}$, a vertex $u_{j} \in U_{j}^{\text {good }}$, and an edge $\left\{u_{j}, v\right\} \in E$.

(1) If $v \in U_{j}$ or $v \in I_{j+1}^{\mathrm{bad}}$, then $c\left(\left\{u_{j}, v\right\}\right)=c_{u_{j}}$ is the primary color of $u_{j}$.

(2) The edges $E_{G}\left(u_{j}, U_{j-1}\right)$ admit at least $\operatorname{deg}_{G}^{c}\left(u_{j}\right)-1-\left|U_{j+1} \backslash I_{j+1}^{\text {bad }}\right|$ non-c $c_{u_{j}}$ colors. 
(3) If $v \in U_{j-1}^{\text {bad }}$ and $c\left(\left\{u_{j}, v\right\}\right) \neq c_{u_{j}}$, then $\operatorname{deg}_{G}^{c}\left(v, U_{j}\right) \geq\left((1 / 6)-37 \lambda^{1 / 4}\right) n$.

(4) If $v \in E_{j-1}^{\mathrm{bad}}$ and $c\left(\left\{u_{j}, v\right\}\right) \neq c_{u_{j}}$, then $\operatorname{deg}_{G}^{c}\left(v, U_{j}\right) \geq \operatorname{deg}_{G}^{c}(v)-3$.

(5) If $v \in U_{j-1}^{\text {good }}$ and $c\left(\left\{u_{j}, v\right\}\right) \neq c_{u_{j}}$, then $\operatorname{deg}_{G}^{c}\left(v, U_{j} \backslash I_{j}^{\text {bad }}\right) \geq \operatorname{deg}_{G}^{c}(v)-1$.

Proof of Corollary 8.1. Let $\ell \equiv 1(\bmod 3)$. Fix an index $j \in \mathbb{Z}_{3}$, and w.l.o.g. let $j=0 \in \mathbb{Z}_{3}$. Fix a good vertex $u_{0} \in U_{0}^{\text {good }}$, and fix an edge $\left\{u_{0}, v\right\} \in E$.

For Statement (1), assume first that $v \in U_{0}$. If $c\left(\left\{u_{0}, v\right\}\right) \neq c_{u_{0}}$, then $P=\left(u_{0}, v\right)$ is a $c_{u_{0}}$-free rainbow path which Proposition 7.1 extends to a strong rainbow $\ell$-cycle $C_{\ell}$ (using $i=j=0, k=2$, and $K=\ell \equiv 1(\bmod 3))$, contradicting (67). Assume next that $v \in I_{1}^{\text {bad }}$, in which case $v$ sees at least three colors in $U_{1}$. If $c\left(\left\{u_{0}, v\right\}\right) \neq c_{u_{0}}$, then $v$ admits a neighbor $w_{1} \in U_{1}$ where $c\left(\left\{v, w_{1}\right\}\right)$ is neither $c_{u_{0}}$ nor $c\left(\left\{u_{0}, v\right\}\right)$. Now, $P=\left(u_{0}, v, w_{1}\right)$ is a $c_{u_{0}}$-free rainbow path which Proposition 7.1 extends to a strong rainbow $\ell$-cycle $C_{\ell}$ (using $i=0, j=1, k=3$, and $K=\ell \equiv 1(\bmod 3)$ ), again contradicting (67).

Statement (2) is an easy consequence of Statement (1). For $u_{0} \in U_{0}^{\text {good }}$ satisfies

$$
\operatorname{deg}_{G}^{c}\left(u_{0}\right)=\operatorname{deg}_{G}^{c}\left(u_{0}, U_{0}\right)+\operatorname{deg}_{G}^{c}\left(u_{0}, U_{1}\right)+\operatorname{deg}_{G}^{c}\left(u_{0}, U_{2}\right),
$$

where Statement (1) guarantees that all edges of $E_{G}\left(u_{0}, U_{0} \cup I_{1}^{\text {bad }}\right)$ are colored $c_{u_{0}}$. However colors are assigned to $E_{G}\left(u_{0}, U_{1} \backslash I_{1}^{\mathrm{bad}}\right)$, at least $\operatorname{deg}_{G}^{c}\left(u_{0}\right)-1-\left|U_{1} \backslash I_{1}^{\mathrm{bad}}\right|$ many non- $c_{u_{0}}$ colors remain, and these must occur on the edges of $E_{G}\left(u_{0}, U_{2}\right)$.

For Statement (3), we prepare an observation used multiple times below. For an edge $\left\{u_{0}, u_{2}\right\} \in$ $E_{G}\left(u_{0}, U_{2}\right)$ satisfying $c\left(\left\{u_{0}, u_{2}\right\}\right) \neq c_{u_{0}}$, we observe that

$$
\text { every edge }\left\{u_{2}, u_{1}\right\} \in E_{G}\left(u_{2}, U_{1}\right) \text { must be colored either } c\left(\left\{u_{0}, u_{2}\right\}\right) \text { or } c_{u_{0}} \text {, }
$$

lest $\left(u_{0}, u_{2}, u_{1}\right)$ is a $c_{u_{0}}$-free rainbow path which Proposition 7.1 extends to a strong rainbow $\ell$-cycle $C_{\ell}$ (using $i=0, j=1, k=3$, and $K=\ell \equiv 1(\bmod 3)$ ), contradicting (67). Now, as in Statement (3), assume $v \in U_{2}^{\text {bad }}$ where $c\left(\left\{u_{0}, v\right\}\right) \neq c_{u_{0}}$. Then (104) gives $\operatorname{deg}_{G}^{c}\left(v, U_{1}^{\text {good }}\right) \leq 2$, where (73) and (74) add that $v \in U_{2}^{\text {bad }}$ satisfies

$$
\operatorname{deg}_{G}^{c}\left(v, U_{0}^{\text {good }}\right) \geq \max \left\{\operatorname{deg}_{G}^{c}\left(v, U_{1}^{\text {good }}\right), \operatorname{deg}_{G}^{c}\left(v, U_{2}^{\text {good }}\right)\right\} \geq \operatorname{deg}_{G}^{c}\left(v, U_{2}^{\text {good }}\right) .
$$

Since $V=U_{1}^{\text {good }} \cup U_{2}^{\text {good }} \cup U_{3}^{\text {good }} \cup V^{\text {bad }}$ is a partition,

$$
\begin{gathered}
\operatorname{deg}_{G}^{c}(v)=\operatorname{deg}_{G}^{c}\left(v, U_{0}^{\text {good }}\right)+\operatorname{deg}_{G}^{c}\left(v, U_{1}^{\text {good }}\right)+\operatorname{deg}_{G}^{c}\left(v, U_{2}^{\text {good }}\right)+\operatorname{deg}_{G}^{c}\left(v, V^{\text {bad }}\right) \\
\leq \operatorname{deg}_{G}^{c}\left(v, U_{0}^{\text {good }}\right)+\operatorname{deg}_{G}^{c}\left(v, U_{1}^{\text {good }}\right)+\operatorname{deg}_{G}^{c}\left(v, U_{2}^{\text {good }}\right)+\left|V^{\text {bad }}\right| \\
\stackrel{104}{\leq} \operatorname{deg}_{G}^{c}\left(v, U_{0}^{\text {good }}\right)+2+\operatorname{deg}_{G}^{c}\left(v, U_{2}^{\text {good }}\right)+\left|V^{\text {bad }}\right| \stackrel{\text { 774 }}{\leq} 2 \operatorname{deg}_{G}^{c}\left(v, U_{0}^{\text {good }}\right)+2+\left|V^{\text {bad }}\right| \\
\stackrel{775}{\leq} 2 \operatorname{deg}_{G}^{c}\left(v, U_{0}^{\text {good }}\right)+2+72 \lambda^{1 / 4} n \leq 2 \operatorname{deg}_{G}^{c}\left(v, U_{0}^{\text {good }}\right)+73 \lambda^{1 / 4} n .
\end{gathered}
$$

Thus, we conclude Statement (3) from

$$
\begin{aligned}
\operatorname{deg}_{G}^{c}\left(v, U_{0}\right) \geq \operatorname{deg}_{G}^{c}\left(v, U_{0}^{\text {good }}\right) \stackrel{105}{\geq} & \frac{1}{2}\left(\operatorname{deg}_{G}^{c}(v)-73 \lambda^{1 / 4} n\right) \\
& \geq \frac{1}{2}\left(\delta^{c}(G)-73 \lambda^{1 / 4} n\right) \geq \frac{1}{2}\left(\frac{n+4}{3}-73 \lambda^{1 / 4} n\right) \geq\left(\frac{1}{6}-37 \lambda^{1 / 4}\right) n .
\end{aligned}
$$

For Statement (4), assume that $v \in E_{2}^{\text {bad }}$ and that $c\left(\left\{u_{0}, v\right\}\right) \neq c_{u_{0}}$. As before with (103), we have

$$
\operatorname{deg}_{G}^{c}(v)=\operatorname{deg}_{G}^{c}\left(v, U_{0}\right)+\operatorname{deg}_{G}^{c}\left(v, U_{1}\right)+\operatorname{deg}_{G}^{c}\left(v, U_{2}\right) .
$$

By the definition of $E_{2}^{\text {bad }}$ in (102), we have $\operatorname{deg}_{G}^{c}\left(v, U_{2}\right) \leq 2$. Moreover, (104) gives $\operatorname{deg}_{G}\left(v, U_{1}\right) \leq 2$, where these colors can only be $c\left(\left\{u_{0}, v\right\}\right)$ and $c_{u_{0}}$. Since $c\left(\left\{u_{0}, v\right\}\right)$ is used on $E_{G}\left(v, U_{0}\right)$, Statement (4) follows.

For Statement (5), assume that $v \in U_{2}^{\text {good }}$ and that $c\left(\left\{u_{0}, v\right\}\right) \neq c_{u_{0}}$. As before with (103), we have

$$
\operatorname{deg}_{G}^{c}(v)=\operatorname{deg}_{G}^{c}\left(v, U_{0}\right)+\operatorname{deg}_{G}^{c}\left(v, U_{1}\right)+\operatorname{deg}_{G}^{c}\left(v, U_{2}\right) .
$$


Statement (1) ensures that all edges of $E_{G}\left(v, U_{2} \cup I_{0}^{\text {bad }}\right)$ are assigned the primary color $c_{v}$. Moreover, (104) gives all edges of $E_{G}\left(v, U_{1}\right)$ are assigned $c\left(\left\{u_{0}, v\right\}\right)$ and $c_{u_{0}}$, which must include $c_{v}$. Now, all non- $\left\{c_{u_{0}}, c_{v}\right\}$ colors incident to $v$ must be on the edges $E_{G}\left(v, U_{0} \backslash I_{0}^{\mathrm{bad}}\right)$, where $c\left(\left\{u_{0}, v\right\}\right)$ is one such color used. In either case of $c_{v} \in\left\{c_{u_{0}}, c\left(\left\{u_{0}, v\right\}\right)\right\}$, only $c_{u_{0}}$ is possibly not used on $E_{G}\left(v, U_{0} \backslash I_{0}^{\text {bad }}\right)$.

8.1. On 4 -cycles $\boldsymbol{C}_{\boldsymbol{4}}$ in $(\boldsymbol{G}, \boldsymbol{c})$ when $\boldsymbol{\ell} \equiv \mathbf{1}(\bmod 3)$. Since $4 \equiv 1(\bmod 3)$, which is the modular case of Lemma 2.9 we seek to prove, we study 4-cycles $C_{4}$ in $(G, c)$ from the point of view of Proposition 7.1 and Corollary 8.1. We begin with the following notation and terminology. For $j \in \mathbb{Z}_{3}$ and $u_{j} \in U_{j}$, recall from (78) that an edge $\left\{u_{j}, u_{j-1}\right\} \in E_{G}\left(u_{j}, U_{j-1}\right)$ is said to be typical when $c\left(\left\{u_{j}, u_{j-1}\right\}\right)=c_{u_{j}}$ is the primary color of $u_{j}$, and is said to be special otherwise. In the reverse of (78), we write

$$
\begin{gathered}
N_{G}^{\text {typ }}\left(u_{j-1}, U_{j}^{\text {good }}\right)=\left\{u_{j} \in N_{G}\left(u_{j-1}, U_{j}^{\text {good }}\right): c\left(\left\{u_{j-1}, u_{j}\right\}\right)=c_{u_{j}}\right\}, \\
N_{G}^{\text {spec }}\left(u_{j-1}, U_{j}^{\text {good }}\right)=\left\{u_{j} \in N_{G}\left(u_{j-1}, U_{j}^{\text {good }}\right): c\left(\left\{u_{j-1}, u_{j}\right\}\right) \neq c_{u_{j}}\right\}, \\
\operatorname{deg}_{G}^{\text {typ }}\left(u_{j-1}, U_{j}^{\text {good }}\right)=\left|N_{G}^{\text {typ }}\left(u_{j-1}, U_{j}^{\text {good }}\right)\right|, \quad \text { and } \quad \operatorname{deg}_{G}^{\text {spec }}\left(u_{j-1}, U_{j}^{\text {good }}\right)=\left|N_{G}^{\text {spec }}\left(u_{j-1}, U_{j}^{\text {good }}\right)\right| .
\end{gathered}
$$

We proceed with an initial observation.

Observation 8.2. Let $\ell \equiv 1(\bmod 3)$. Fix $j \in \mathbb{Z}_{3}, u_{j-1} \in U_{j-1}$, and $u_{j} \neq v_{j} \in N_{G}^{\text {spec }}\left(u_{j-1}, U_{j}^{\text {good }}\right)$. Then $c\left(\left\{u_{j-1}, u_{j}\right\}\right), c\left(\left\{u_{j-1}, v_{j}\right\}\right), c_{u_{j}}$, and $c_{v_{j}}$ can't all be distinct.

Proof of Observation 8.2. Let $\ell \equiv 1(\bmod 3)$. Fix $j \in \mathbb{Z}_{3}$, and w.l.o.g. let $j=0$. Fix $u_{2} \in U_{2}$ and fix $u_{0} \neq v_{0} \in N_{G}^{\text {spec }}\left(u_{2}, U_{0}^{\text {good }}\right)$. We apply Proposition 6.2 and Corollary 6.3 to each of $u_{0} \neq v_{0} \in U_{0}^{\text {good }}$ to determine at least

$$
\left|U_{2}^{\text {good }}\right|-322 \lambda^{1 / 4} n \stackrel{75}{\geq}\left(\frac{1}{3}-397 \lambda^{1 / 4}\right) n \stackrel{65}{>} 0
$$

many vertices $w_{2} \in U_{2}^{\text {good }}$ for which $\left\{u_{0}, w_{2}\right\},\left\{v_{0}, w_{2}\right\} \in E$ and

$$
c\left(\left\{u_{0}, w_{2}\right\}\right)=c_{u_{0}} \neq c_{v_{0}}=c\left(\left\{v_{0}, w_{2}\right\}\right) .
$$

If Observation 8.2 is false, then $\left(u_{0}, w_{2}, v_{0}, u_{2}\right)$ is a strong rainbow 4-cycle which Statement (3) of Proposition 7.1 extends to a strong rainbow $\ell$-cycle $C_{\ell}$, contradicting (667).

We continue with a second observation, which is a corollary of the one above.

Corollary 8.3. Let $\ell \equiv 1(\bmod 3)$. Fix $j \in \mathbb{Z}_{3}$ and $u_{j-1} \in U_{j-1}$. If $\operatorname{deg}_{G}^{\text {spec }}\left(u_{j-1}, U_{j}^{\text {good }}\right) \geq 4$, then all but at most one of the edges $\left\{u_{j-1}, u_{j}\right\} \in E$, where $u_{j} \in N_{G}^{\text {spec }}\left(u_{j-1}, U_{j}^{\text {good }}\right)$ are monochromatic. Thus,

$$
\operatorname{deg}_{G}^{\text {typ }}\left(u_{j-1}, U_{j}^{\text {good }}\right) \geq \operatorname{deg}_{G}^{c}\left(u_{j-1}, U_{j}^{\text {good }}\right)-3,
$$

where in particular

$$
\operatorname{deg}_{G}^{\text {typ }}\left(u_{j-1}, U_{j}^{\text {good }}\right) \geq\left\{\begin{array}{cc}
\left(\frac{1}{6}-110 \lambda^{1 / 4}\right) n & \text { if } \operatorname{deg}_{G}^{\text {spec }}\left(u_{j-1}, U_{j}^{\text {good }}\right) \geq 1, \\
\left(\frac{1}{9}-144 \lambda^{1 / 4}\right) n & \text { otherwise. }
\end{array}\right.
$$

Proof of Corollary 8.3. Let $\ell \equiv 1(\bmod 3)$. Fix $j \in \mathbb{Z}_{3}$ and w.l.o.g. assume $j=0$. Fix $u_{2} \in U_{2}$ and assume that $\operatorname{deg}_{G}^{\mathrm{spec}}\left(u_{2}, U_{0}^{\text {good }}\right) \geq 4$. For sake of argument,

we assume the special edges $\left\{u_{2}, u_{0}\right\} \in E$,

$$
\text { where } u_{0} \in N_{G}^{\text {spec }}\left(u_{2}, U_{0}^{\text {good }}\right) \text {, are not entirely monochromatic. }
$$

We consider two cases. 
Case $1\left(\exists u_{0} \neq v_{0} \in N_{G}^{\text {spec }}\left(u_{2}, U_{0}^{\text {good }}\right): c\left(\left\{u_{2}, u_{0}\right\}\right)=c\left(\left\{u_{2}, v_{0}\right\}\right)\right)$. Fix $u_{0} \neq v_{0} \in N_{G}^{\text {spec }}\left(u_{2}, U_{0}^{\text {good }}\right)$ with $c\left(\left\{u_{2}, u_{0}\right\}\right)=c\left(\left\{u_{2}, v_{0}\right\}\right)$. Using (106), we infer the existence of an edge $\left\{u_{2}, w_{0}\right\} \in E$, where $w_{0} \in N_{G}^{\text {spec }}\left(u_{2}, U_{0}^{\text {good }}\right)$, for which $c\left(\left\{u_{2}, w_{0}\right\}\right) \neq c\left(\left\{u_{2}, u_{0}\right\}\right)=c\left(\left\{u_{2}, v_{0}\right\}\right)$. We claim that

$$
c\left(\left\{u_{2}, u_{0}\right\}\right)=c\left(\left\{u_{2}, v_{0}\right\}\right)=c_{w_{0}} .
$$

Indeed, pivoting $\left\{u_{2}, u_{0}\right\}$ against $\left\{u_{2}, w_{0}\right\}$, Observation 8.2 ensures $c\left(\left\{u_{2}, u_{0}\right\}\right)=c_{w_{0}}$ or $c\left(\left\{u_{2}, w_{0}\right\}\right)=$ $c_{u_{0}}$. In the former case, (107) holds by the hypothesis of Case 1. In the latter case, we pivot $\left\{u_{2}, v_{0}\right\}$ against $\left\{u_{2}, w_{0}\right\}$, where Observation 8.2 gives $c\left(\left\{u_{2}, v_{0}\right\}\right)=c_{w_{0}}$ or $c\left(\left\{u_{2}, w_{0}\right\}\right)=c_{v_{0}}$. If $c\left(\left\{u_{2}, w_{0}\right\}\right)=c_{u_{0}}$, then Corollary 6.3 gives $c\left(\left\{u_{2}, w_{0}\right\}\right) \neq c_{v_{0}}$, and so $c\left(\left\{u_{2}, v_{0}\right\}\right)=c_{w_{0}}$ and again (107) holds.

If the first conclusion of Corollary 8.3 does not hold, we ignore the edge $\left\{u_{2}, w_{0}\right\}$ to infer the existence of an edge $\left\{u_{2}, x_{0}\right\} \in E$, where $x_{0} \in N_{G}^{\text {spec }}\left(u_{2}, U_{0}^{\text {good }}\right)$, for which $c\left(\left\{u_{2}, x_{0}\right\}\right) \neq c\left(\left\{u_{2}, u_{0}\right\}\right)$. By (107),

$$
c_{x_{0}}=c\left(\left\{u_{2}, u_{0}\right\}\right)=c\left(\left\{u_{2}, v_{0}\right\}\right)=c_{w_{0}},
$$

and $x_{0} \neq w_{0} \in U_{0}^{\text {good }}$ contradicts Corollary 6.3

Case $2\left(\forall u_{0} \neq v_{0} \in N_{G}^{\text {spec }}\left(u_{2}, U_{0}^{\text {good }}\right), c\left(\left\{u_{2}, u_{0}\right\}\right) \neq c\left(\left\{u_{2}, v_{0}\right\}\right)\right)$. Since $\operatorname{deg}_{G}^{\text {spec }}\left(u_{2}, U_{0}^{\text {good }}\right) \geq 4$, fix distinct $u_{0}, v_{0}, w_{0}, x_{0} \in N_{G}^{\text {spec }}\left(u_{2}, U_{0}^{\text {good }}\right)$. Using Observation 8.2, we take w.l.o.g. $c\left(\left\{u_{2}, u_{0}\right\}\right)=c_{v_{0}}$. Observation 8.2 then ensures that $c\left(\left\{u_{2}, w_{0}\right\}\right)=c_{u_{0}}$ (since $c_{v_{0}} \neq c_{w_{0}}$ from Corollary6.3) and $c\left(\left\{u_{2}, x_{0}\right\}\right)=c_{u_{0}}$, which contradicts the hypothesis of Case 2 .

The remaining assertions of Corollary 8.3 are now easy to establish. When $\operatorname{deg}_{G}^{\text {spec }}\left(u_{2}, U_{0}^{\text {good }}\right)=0$, all edges of $E_{G}\left(u_{2}, U_{0}^{\text {good }}\right)$ are typical, and so

$$
\operatorname{deg}_{G}^{\text {typ }}\left(u_{2}, U_{0}^{\text {good }}\right) \geq \operatorname{deg}_{G}^{c}\left(u_{2}, U_{0}^{\text {good }}\right) \stackrel{75}{\geq}\left(\frac{1}{9}-72 \lambda^{1 / 4}\right) n-72 \lambda^{1 / 4} n=\left(\frac{1}{9}-144 \lambda^{1 / 4}\right) n .
$$

When $\operatorname{deg}_{G}^{\text {spec }}\left(u_{2}, U_{0}^{\text {good }}\right) \geq 1$, the first assertion of Corollary 8.3 guarantees that edges $\left\{u_{2}, u_{0}\right\} \in E$ with $u_{0} \in N_{G}^{\text {spec }}\left(u_{2}, U_{0}^{\text {good }}\right)$ are colored with at most three colors. Thus,

$$
\begin{array}{r}
\operatorname{deg}_{G}^{\text {typ }}\left(u_{2}, U_{0}^{\text {good }}\right) \geq \operatorname{deg}_{G}^{c}\left(u_{2}, U_{0}^{\text {good }}\right)-3 \stackrel{\text { Cor } 8.1}{\geq}\left(\frac{1}{6}-37 \lambda^{1 / 4}\right) n-72 \lambda^{1 / 4} n-3 \\
=\left(\frac{1}{6}-109 \lambda^{1 / 4}\right) n-3 \geq\left(\frac{1}{6}-110 \lambda^{1 / 4}\right) n,
\end{array}
$$

as promised.

Definition 8.4 ( $j$-special). A 4-cycle $\left(u_{j}, u_{j-1}, v_{j}, v_{j-1}\right)$ is $j$-special for some $j \in \mathbb{Z}_{3}$ if $u_{j}, v_{j} \in U_{j}^{\text {good }}$, $u_{j-1}, v_{j-1} \in U_{j-1},\left\{u_{j}, u_{j-1}\right\},\left\{v_{j}, v_{j-1}\right\} \in E$ are typical, and $\left\{u_{j}, v_{j-1}\right\},\left\{v_{j}, u_{j-1}\right\} \in E$ are special.

Observation 8.5. Let $\ell \equiv 1(\bmod 3)$, and fix $j \in \mathbb{Z}_{3} . A j$-special 4 -cycle $\left(u_{j}, u_{j-1}, v_{j}, v_{j-1}\right)$ receives precisely three colors, where in particular $c\left(\left\{u_{j}, v_{j-1}\right\}\right)=c\left(\left\{v_{j}, u_{j-1}\right\}\right)$.

Proof of Observation 8.5, Let $\ell \equiv 1(\bmod 3)$. Fix $j \in \mathbb{Z}_{3}$, and w.l.o.g. let $j=0$. Fix a 0-special 4cycle $\left(u_{0}, u_{2}, v_{0}, v_{2}\right)$. By its definition, we infer $c\left(\left\{u_{2}, u_{0}\right\}\right)=c_{u_{0}}$ and $c\left(\left\{v_{2}, v_{0}\right\}\right)=c_{v_{0}}$ are primary, which Corollary 6.3 ensures are distinct. By its definition, $c\left(\left\{u_{0}, v_{2}\right\}\right) \neq c_{u_{0}}$ and $c\left(\left\{v_{0}, u_{2}\right\}\right) \neq c_{v_{0}}$ are special. Observe that $c\left(\left\{u_{0}, v_{2}\right\}\right) \neq c_{v_{0}}$ since otherwise Proposition 6.2 guarantees that $G-\left\{v_{0}, v_{2}\right\}$ (here denoting edge-removal) contradicts (68). Similarly, $c\left(\left\{v_{0}, u_{2}\right\}\right) \neq c_{u_{0}}$. Thus, if $c\left(\left\{u_{0}, v_{2}\right\}\right) \neq c\left(\left\{v_{0}, u_{2}\right\}\right)$, then $\left(u_{0}, u_{2}, v_{0}, v_{2}\right)$ is a strong rainbow 4-cycle which Statement (3) of Proposition 7.1 extends to a strong rainbow $\ell$-cycle $C_{\ell}$, contradicting (67).

We conclude this subsection with a corollary of the preceding observation.

Corollary 8.6. Let $\ell \equiv 1(\bmod 3)$. Fix $j \in \mathbb{Z}_{3}, u_{j-1} \neq v_{j-1} \in U_{j-1}$, and a color $\alpha$ from c. Set

$$
\begin{aligned}
A= & A_{\alpha}\left(u_{j-1}\right)=\left\{u_{j} \in N_{G}^{\mathrm{spec}}\left(u_{j-1}, U_{j}\right): c\left(\left\{u_{j}, u_{j-1}\right\}\right)=\alpha\right\} \\
& \text { and } B=B_{\alpha}\left(v_{j-1}\right)=\left\{v_{j} \in N_{G}^{\mathrm{spec}}\left(v_{j-1}, U_{j}\right): c\left(\left\{v_{j}, v_{j-1}\right\}\right) \neq \alpha\right\} .
\end{aligned}
$$

Then $|A \cup B|<\left((1 / 6)+186 \lambda^{1 / 4}\right) n$. 
Proof of Corollary 8.6. Let $\ell \equiv 1(\bmod 3)$. Fix $j \in \mathbb{Z}_{3}$, and w.l.o.g. let $j=0$. Fix $u_{2} \neq v_{2} \in U_{2}$ and fix a color $\alpha$ of $c$. Let $A=A\left(u_{2}\right)$ and $B=B\left(v_{2}\right)$ be defined as above, but assume for contradiction that

$$
|A \cup B| \geq\left(\frac{1}{6}+186 \lambda^{1 / 4}\right) n .
$$

We will use (108) to guarantee distinct vertices

$$
N_{G}^{\text {typ }}\left(u_{2}, U_{0}^{\text {good }}\right) \cap(A \cup B) \ni u_{0} \neq v_{0} \in N_{G}^{\text {typ }}\left(v_{2}, U_{0}^{\text {good }}\right) \cap(A \cup B) .
$$

If (109) holds, then it will conclude our proof, because $\left(u_{2}, u_{0}, v_{2}, v_{0}\right)$ would be a rainbow 0-special 4cycle, contradicting Observation 8.5. To see this, we first note from (109) that $u_{0} \neq v_{0} \in U_{0}^{\text {good }}$ are good vertices, where $u_{0} \in N_{G}^{\text {typ }}\left(u_{2}, U_{0}^{\text {good }}\right)$ guarantees $c\left(\left\{u_{0}, u_{2}\right\}\right)=c_{u_{0}}$ and $v_{0} \in N_{G}^{\text {typ }}\left(v_{2}, U_{0}^{\text {good }}\right)$ guarantees $c\left(\left\{v_{0}, v_{2}\right\}\right)=c_{v_{0}}$, and where $c_{u_{0}} \neq c_{v_{0}}$ is guaranteed by Corollary 6.3. Since $u_{0} \in A \cup B$ happens only from $u_{0} \in B \backslash A$ (because $c\left(\left\{u_{0}, u_{2}\right\}\right)=c_{u_{0}}$ is not special for $\left.u_{0}\right)$, we infer $c\left(\left\{u_{0}, v_{2}\right\}\right) \neq \alpha$ is some non- $\alpha$ special color for $u_{0}$. Since $v_{0} \in A \cup B$ happens only from $v_{0} \in A \backslash B$ (because $c\left(\left\{v_{0}, v_{2}\right\}\right)=c_{v_{0}}$ is not special for $\left.v_{0}\right)$, we infer $c\left(\left\{v_{0}, u_{2}\right\}\right)=\alpha$ is special for $v_{0}$. (Note: the existence of vertices $u_{0}$ and $v_{0}$ in (109) implies they are necessarily distinct.) Thus, $\left(u_{2}, u_{0}, v_{2}, v_{0}\right)$ is a rainbow 0 -special 4 -cycle, as claimed.

To prove (109) (from (108)), define

$$
A^{\text {good }}=A \cap U_{0}^{\text {good }} \quad \text { and } \quad B^{\text {good }}=B \cap U_{0}^{\text {good }} .
$$

Then

$$
\left|A^{\text {good }} \cup B^{\text {good }}\right| \stackrel{\text { 755 }}{\geq}|A \cup B|-72 \lambda^{1 / 4} n \stackrel{108}{\geq}\left(\frac{1}{6}+114 \lambda^{1 / 4}\right) n
$$

and so one of $\left|A^{\text {good }}\right|$ or $\left|B^{\text {good }}\right|$ is large. Our proof will ultimately show that both are large, and so we begin by assuming the former is non-empty. We therefore infer that $\operatorname{deg}_{G}^{\text {spec }}\left(u_{2}, U_{0}^{\text {good }}\right) \geq\left|A^{\text {good }}\right|>0$, and so Corollary 8.3 gives

$$
\operatorname{deg}_{G}^{\text {typ }}\left(u_{2}, U_{0}^{\text {good }}\right) \geq\left(\frac{1}{6}-110 \lambda^{1 / 4}\right) n .
$$

If $N_{G}^{\text {typ }}\left(u_{2}, U_{0}^{\text {good }}\right)$ and $A \cup B$ were disjoint, we would have

$$
\begin{aligned}
\left|U_{0}\right| \geq \operatorname{deg}_{G}^{\text {typ }}\left(u_{2}, U_{0}^{\text {good }}\right)+ & |A \cup B| \stackrel{111}{\geq}\left(\frac{1}{6}-110 \lambda^{1 / 4}\right) n+|A \cup B| \\
& \frac{(108)}{\geq}\left(\frac{1}{6}-110 \lambda^{1 / 4}\right) n+\left(\frac{1}{6}+186 \lambda^{1 / 4}\right) n=\left(\frac{1}{3}+76 \lambda^{1 / 4}\right) n \stackrel{\sqrt[75]{>}}{>}\left|U_{0}\right|,
\end{aligned}
$$

a contradiction. This guarantees the existence of the vertex $u_{0}$ in (109).

To guarantee the existence of the vertex $v_{0}$ in (109), we argue similarly. For that, $N_{G}^{\text {typ }}\left(u_{2}, U_{0}^{\text {good }}\right)$ and $A^{\text {good }}$ are disjoint subsets of $U_{0}^{\text {good }}$, and so

$$
\begin{aligned}
& \left|A^{\text {good }}\right|+\left(\frac{1}{6}-110 \lambda^{1 / 4}\right) n \stackrel{111}{\leq}\left|A^{\text {good }}\right|+\operatorname{deg}_{G}^{\text {typ }}\left(u_{2}, U_{0}^{\text {good }}\right) \leq\left|U_{0}^{\text {good }}\right| \\
& \stackrel{755}{\leq}\left(\frac{1}{3}+75 \lambda^{1 / 4}\right) n \quad \Longrightarrow \quad\left|A^{\text {good }}\right| \leq\left(\frac{1}{6}+185 \lambda^{1 / 4}\right) n .
\end{aligned}
$$

Thus,

$$
\begin{aligned}
\operatorname{deg}_{G}^{\text {spec }}\left(v_{2}, U_{0}^{\text {good }}\right) \geq\left|B^{\text {good }} \backslash A^{\text {good }}\right|=\left|A^{\text {good }} \cup B^{\text {good }}\right|-\left|A^{\text {good }}\right| & \\
& \frac{110}{\geq}\left(\frac{1}{6}+186 \lambda^{1 / 4}\right) n-\left|A^{\text {good }}\right| \stackrel{(113)}{\geq} \lambda^{1 / 4} n>0,
\end{aligned}
$$

and so Corollary 8.3 gives

$$
\operatorname{deg}_{G}^{\text {typ }}\left(v_{2}, U_{0}^{\text {good }}\right) \geq\left(\frac{1}{6}-110 \lambda^{1 / 4}\right) n \text {. }
$$

We now proceed identically to before with (111)-(112). 
8.2. Amenable elements of $\mathbb{Z}_{3}$. Recall the partition $V(G)=U_{0} \cup U_{1} \cup U_{2}$ of $(G, c)$ from (75)). For each $j \in \mathbb{Z}_{3}$, recall the (so-called internal and external) sets of bad vertices

$$
I_{j}^{\mathrm{bad}}=\left\{u_{j} \in U_{j}^{\mathrm{bad}}: \operatorname{deg}_{G}^{c}\left(u_{j}, U_{j}\right) \geq 3\right\} \quad \text { and } \quad E_{j}^{\mathrm{bad}}=\left\{u_{j} \in U_{j}^{\mathrm{bad}}: \operatorname{deg}_{G}^{c}\left(u_{j}, U_{j}\right) \leq 2\right\}
$$

from (102). In particular, $U_{j}^{\mathrm{bad}}=I_{j}^{\mathrm{bad}} \cup E_{j}^{\mathrm{bad}}$ is a partition, and so

$$
U_{j}=U_{j}^{\mathrm{good}} \cup U_{j}^{\mathrm{bad}}=U_{j}^{\mathrm{good}} \cup I_{j}^{\mathrm{bad}} \cup E_{j}^{\mathrm{bad}}
$$

are partitions. We set

$$
\hat{U}_{j}=U_{j}^{\text {good }} \cup E_{j}^{\text {bad }} \quad \text { and } \quad \Delta_{j}=m-\left|\hat{U}_{j}\right|,
$$

where $m=\lfloor n / 3\rfloor$ from (69). The following observation follows by elementary means (independent from $\ell(\bmod 3))$, and plays an important role in our proof of Lemma 2.9 .

Observation 8.7. There exists $j \in \mathbb{Z}_{3}$ so that

(1) $\Delta_{j} \geq 0$

(2) $\left|I_{j+1}^{\mathrm{bad}}\right| \leq 2 \Delta_{j}$

(3) $\left|U_{j+2}\right| \leq m+2 \Delta_{j}+2$.

We say that an element $j \in \mathbb{Z}_{3}$ satisfying Conclusions (1)-(3) of Observation 8.7 is amenable.

Proof of Observation 8.7. As they are defined in (115), let $j \in \mathbb{Z}_{3}$ satisfy

$$
\Delta_{j}=\max \left\{\Delta_{0}, \Delta_{1}, \Delta_{2}\right\}, \quad \text { and w.l.o.g. let } j=0 .
$$

Conclusion (1) holds with $j=0$ from (116), lest $\Delta_{1}, \Delta_{2} \leq \Delta_{0} \leq-1$ and $m=\lfloor n / 3\rfloor \geq(n-2) / 3$ give

$$
\begin{aligned}
3 m-\left|\hat{U}_{0}\right|-\left|\hat{U}_{1}\right| & -\left|\hat{U}_{2}\right| \stackrel{115}{=} \Delta_{0}+\Delta_{1}+\Delta_{2} \leq-3 \\
& \Longrightarrow \quad 3 m+3 \leq\left|\hat{U}_{0}\right|+\left|\hat{U}_{1}\right|+\left|\hat{U}_{2}\right| \stackrel{114}{\leq}\left|U_{0}\right|+\left|U_{1}\right|+\left|U_{2}\right|=n \leq 3 m+2,
\end{aligned}
$$

a contradiction. Conclusion (3) also holds with $j=0$ from (116), since

$$
\begin{array}{r}
\left|U_{2}\right|=n-\left|U_{0}\right|-\left|U_{1}\right| \stackrel{114,,[15)}{=} n-\left|I_{0}^{\mathrm{bad}}\right|-\left|\hat{U}_{0}\right|-\left|I_{1}^{\mathrm{bad}}\right|-\left|\hat{U}_{1}\right| \leq n-\left|\hat{U}_{0}\right|-\left|\hat{U}_{1}\right| \\
\leq m+2+\left(m-\left|\hat{U}_{0}\right|\right)+\left(m-\left|\hat{U}_{1}\right|\right) \stackrel{115}{=} m+2+\Delta_{0}+\Delta_{1} \stackrel{116}{\leq} m+2 \Delta_{0}+2 .
\end{array}
$$

For sake of argument, we assume that Conclusion (2) fails with $j=0$ from (116):

$$
\left|I_{1}^{\text {bad }}\right| \geq 2 \Delta_{0}+1 \text {. }
$$

Observe that

$$
\begin{aligned}
\left|I_{0}^{\mathrm{bad}}\right|+\left|I_{1}^{\mathrm{bad}}\right| \leq\left|I_{0}^{\mathrm{bad}}\right|+\left|I_{1}^{\mathrm{bad}}\right|+\left|I_{2}^{\mathrm{bad}}\right| \stackrel{114 \mathrm{11},[15]}{=} n-\left|\hat{U}_{0}\right|-\left|\hat{U}_{1}\right|-\left|\hat{U}_{2}\right| \\
\leq 2+\left(m-\left|\hat{U}_{0}\right|\right)+\left(m-\left|\hat{U}_{1}\right|\right)+\left(m-\left|\hat{U}_{2}\right|\right) \stackrel{\sqrt[115]{=}}{=} 2+\Delta_{0}+\Delta_{1}+\Delta_{2}, \\
\Longrightarrow \quad 0 \leq\left|I_{0}^{\mathrm{bad}}\right| \leq 2+\Delta_{0}+\Delta_{1}+\Delta_{2}-\left|I_{1}^{\mathrm{bad}}\right| \stackrel{\frac{117}{\leq}}{\leq} 1+\Delta_{1}+\Delta_{2}-\Delta_{0} .
\end{aligned}
$$

If $\Delta_{1} \leq-1$, then Observation 8.7 would hold with $j=2$. Indeed, $\Delta_{1} \leq-1$ and $\Delta_{2} \leq \Delta_{0}$ from (116) would render $I_{0}^{\mathrm{bad}}=\emptyset$, and so $\Delta_{1}=-1$, and $\Delta_{2}=\Delta_{0}$ would be the maximum from (116). If $\Delta_{2}=\Delta_{0}$ is the maximum from (116), we already observed that Conclusion (1) would hold with $j=2$, i.e., $\Delta_{2} \geq 0$, and that Conclusion (3) would hold with $j=2$, i.e., $\left|U_{1}\right| \leq m+2 \Delta_{2}+2$. If $I_{0}^{\text {bad }}=\emptyset$ and $\Delta_{2} \geq 0$, Conclusion (2) would hold with $j=2$, i.e., $\left|I_{0}^{\text {bad }}\right|=0 \leq 2 \Delta_{2}$. For sake of argument, we assume that

$$
\Delta_{1} \geq 0 .
$$


Note that (119) says Conclusion (1) holds with $j=1$. Conclusion (3) also holds with $j=1$, since

$$
\begin{aligned}
& \left|U_{0}\right| \stackrel{114, \underline{115}}{=}\left|\hat{U}_{0}\right|+\left|I_{0}^{\mathrm{bad}}\right| \stackrel{118}{\leq}\left|\hat{U}_{0}\right|+1+\Delta_{1}+\Delta_{2}-\Delta_{0} \\
& \stackrel{116}{\leq}\left|\hat{U}_{0}\right|+1+\Delta_{1} \stackrel{115}{=} m-\Delta_{0}+1+\Delta_{1} \stackrel{116}{\leq} m+1+\Delta_{1} \stackrel{119}{\leq} m+2 \Delta_{1}+2 \text {, }
\end{aligned}
$$

where we used $\Delta_{0} \geq 0$. For sake of argument, we assume Conclusion (2) fails with $j=1$ :

$$
\left|I_{2}^{\text {bad }}\right| \geq 2 \Delta_{1}+1
$$

We conclude that Observation 8.7 holds with $j=2$. For that, observe that

$$
\begin{aligned}
\left|I_{0}^{\mathrm{bad}}\right|+2 \Delta_{0}+2 \Delta_{1}+2 \stackrel{1177, \sqrt[120]{\leq}}{\leq}\left|I_{0}^{\mathrm{bad}}\right|+\left|I_{1}^{\mathrm{bad}}\right|+\left|I_{2}^{\mathrm{bad}}\right| \stackrel{118}{\leq} 2+\Delta_{0}+\Delta_{1}+\Delta_{2} \\
\Longrightarrow \quad 0 \leq\left|I_{0}^{\mathrm{bad}}\right| \leq \Delta_{2}-\Delta_{0}-\Delta_{1} \frac{116}{\leq}-\Delta_{1} \frac{119}{\leq} 0 .
\end{aligned}
$$

Thus, $I_{0}^{\mathrm{bad}}=\emptyset, \Delta_{1}=0$, and $\Delta_{2}=\Delta_{0}$ is the maximum from (116). As $\Delta_{2}=\Delta_{0}$ is the maximum from (116), we already observed that Conclusion (1) holds, i.e., $\Delta_{2} \geq 0$, and that Conclusion (3) holds, i.e., $\left|U_{1}\right| \leq m+2 \Delta_{2}+2$. Since $\Delta_{1}=0$ and $I_{0}^{\text {bad }}=\emptyset$, Conclusion (2) also holds, i.e., $\left|I_{0}^{\text {bad }}\right|=0=2 \Delta_{1}$, which completes the proof of Observation 8.7 .

Observation 8.7 guarantees at least one amenable element $j \in \mathbb{Z}_{3}$, i.e., one where Conclusions (1)-(3) of Observation 8.7 hold. We next consider properties of an amenable $j \in \mathbb{Z}_{3}$ when $\ell \equiv 1(\bmod 3)$.

Fact 8.8. Let $\ell \equiv 1(\bmod 3)$. Let $j \in \mathbb{Z}_{3}$ be amenable, and fix $u_{j+1} \in U_{j+1}$. Then

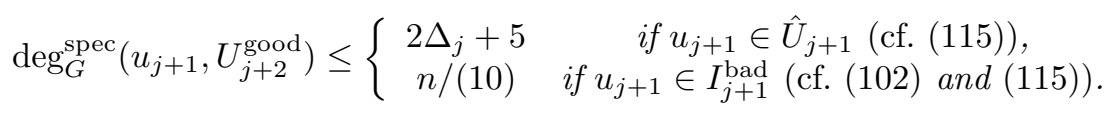

Proof of Fact 8.8. Let $\ell \equiv 1(\bmod 3)$. Fix an amenable $j \in \mathbb{Z}_{3}$, and w.l.o.g. let $j=0$. Note first that

$$
\operatorname{deg}_{G}^{c}\left(v, U_{2}\right) \geq \operatorname{deg}_{G}^{c}(v)-3 \text { for every } v \in \hat{U}_{1} \text { with } \operatorname{deg}_{G}^{\text {spec }}\left(v, U_{2}^{\text {good }}\right)>0,
$$

since $u_{2} \in N_{G}^{\text {spec }}\left(v, U_{2}^{\text {good }}\right)$ gives $u_{2} \in U_{2}^{\text {good }}$ and $c\left(\left\{u_{2}, v\right\}\right) \neq c_{u_{2}}$, and $v \in \hat{U}_{1}=U_{1}^{\text {good }} \cup E_{1}^{\text {bad }}$ allows us to apply Statement (4) or (5) of Corollary 8.1.

Fix a vertex $u_{1} \in U_{1}$, and first let $u_{1} \in \hat{U}_{1}$. Assume, on the contrary, that

$$
\operatorname{deg}_{G}^{\text {spec }}\left(u_{1}, U_{2}^{\text {good }}\right) \geq 2 \Delta_{0}+6 \stackrel{\text { Obs } 8.7}{\geq} 6 .
$$

Now, the edges $E_{G}\left(u_{1}, U_{2}\right)$ consist of $\operatorname{deg}_{G}^{c}\left(u_{1}, U_{2}\right)$ many distinctly colored edges together with some number of edges of repeated colors. By Corollary 8.3 the special edges $E_{G}^{\text {spec }}\left(u_{1}, U_{2}^{\text {good }}\right)$, i.e., those of the form $\left\{u_{1}, u_{2}\right\} \in E$ for $u_{2} \in N_{G}^{\text {spec }}\left(u_{1}, U_{2}^{\text {good }}\right)$, come in at most two colors, so we have

$$
\begin{aligned}
&\left|U_{2}\right| \geq \operatorname{deg}_{G}\left(u_{1}, U_{2}\right) \geq \operatorname{deg}_{G}^{c}\left(u_{1}, U_{2}\right)+\operatorname{deg}_{G}^{\text {spec }}\left(u_{1}, U_{2}^{\text {good }}\right)-2 \\
&\quad 121] \\
& \geq \operatorname{deg}_{G}^{c}\left(u_{1}\right)+\operatorname{deg}_{G}^{\text {spec }}\left(u_{1}, U_{2}^{\text {good }}\right)-5 \stackrel{(122)}{\geq} \operatorname{deg}_{G}^{c}\left(u_{1}\right)+2 \Delta_{0}+1 \stackrel{69}{\geq} m+2 \Delta_{0}+3,
\end{aligned}
$$

which contradicts Conclusion (3) of Observation 8.7

Now let $u_{1} \in I_{1}^{\mathrm{bad}}$. Assume, on the contrary, that

$$
\operatorname{deg}_{G}^{\text {spec }}\left(u_{1}, U_{2}^{\text {good }}\right)>\frac{n}{10} \text {. }
$$

By Corollary 8.3, all but one of the edges $E_{G}^{\text {spec }}\left(u_{1}, U_{2}^{\text {good }}\right)$ are monochromatic, and in some color $\alpha$ of c. To prepare an upcoming application of Corollary [8.6, we define

$$
\begin{aligned}
& A_{2}=A_{2}\left(u_{1}\right)=\left\{v_{2} \in N_{G}^{\text {spec }}\left(u_{1}, U_{2}^{\text {good }}\right)\right.\left.: c\left(\left\{u_{1}, v_{2}\right\}\right)=\alpha\right\}, \\
& \text { where } \quad\left|A_{2}\right| \stackrel{\text { Cor } 8.3}{\geq} \operatorname{deg}_{G}^{\text {spec }}\left(u_{1}, U_{2}^{\text {good }}\right)-1 \stackrel{123}{>} \frac{n}{10}-1 \geq \frac{n}{11} .
\end{aligned}
$$


For Corollary 8.6. we will identify a set $B_{2} \subseteq U_{2}^{\text {good }}$ corresponding to $A_{2}$ above 3 . For that, we first consider the following superset $\bar{B}_{2} \supseteq B_{2}$, from which we will later extract $B_{2}$ :

$$
\bar{B}_{2}=N_{G}^{\text {typ }}\left(u_{1}, U_{2}^{\text {good }}\right), \quad \text { where } \quad\left|\bar{B}_{2}\right| \stackrel{\text { Cor } 8.3}{\geq}\left(\frac{1}{6}-110 \lambda^{1 / 4}\right) n .
$$

For fixed $u_{2} \in \bar{B}_{2}$,

$$
\operatorname{deg}_{G}^{\text {typ }}\left(v_{1}, A_{2}\right)>0 \text { for every } v_{1} \in N_{G}^{\text {spec }}\left(u_{2}, \hat{U}_{1}\right)
$$

since then $\operatorname{deg}_{G}^{\text {spec }}\left(v_{1}, U_{2}^{\text {good }}\right)>0$ (with $\left.u_{2} \in N_{G}^{\text {spec }}\left(v_{1}, U_{2}^{\text {good }}\right)\right)$ and inclusion-exclusion gives

$$
\begin{aligned}
& U_{2}^{\text {good }} \supseteq A_{2} \cup N_{G}^{\text {typ }}\left(v_{1}, U_{2}^{\text {good }}\right) \quad \Longrightarrow \quad\left|U_{2}^{\text {good }}\right| \geq\left|A_{2}\right|+\operatorname{deg}_{G}^{\text {typ }}\left(v_{1}, U_{2}^{\text {good }}\right)-\operatorname{deg}_{G}^{\text {typ }}\left(v_{1}, A_{2}\right) \\
& \Longrightarrow \quad \operatorname{deg}_{G}^{\text {typ }}\left(v_{1}, A_{2}\right) \geq\left|A_{2}\right|+\operatorname{deg}_{G}^{\text {typ }}\left(v_{1}, U_{2}^{\text {good }}\right)-\left|U_{2}^{\text {good }}\right| \stackrel{124}{>} \frac{n}{11}+\operatorname{deg}_{G}^{\text {typ }}\left(v_{1}, U_{2}^{\text {good }}\right)-\left|U_{2}^{\text {good }}\right| \\
& \stackrel{755}{\geq} \frac{n}{11}+\operatorname{deg}_{G}^{\text {typ }}\left(v_{1}, U_{2}^{\text {good }}\right)-\left(\frac{1}{3}+75 \lambda^{1 / 4}\right) n \stackrel{\text { Cor } 8.3}{\geq} \frac{n}{11}+\operatorname{deg}_{G}^{c}\left(v_{1}, U_{2}^{\text {good }}\right)-3-\left(\frac{1}{3}+75 \lambda^{1 / 4}\right) n \\
& \stackrel{750}{\geq} \frac{n}{11}+\operatorname{deg}_{G}^{c}\left(v_{1}, U_{2}\right)-3-\left(\frac{1}{3}+147 \lambda^{1 / 4}\right) n \stackrel{\text { (121) }}{\geq} \frac{n}{11}+\operatorname{deg}_{G}^{c}\left(v_{1}\right)-6-\left(\frac{1}{3}+147 \lambda^{1 / 4}\right) n \\
& \geq \frac{n}{11}+\delta_{c}(G)-6-\left(\frac{1}{3}+147 \lambda^{1 / 4}\right) n \stackrel{66}{\geq} \frac{n}{11}-6-147 \lambda^{1 / 4} n \geq \frac{n}{11}-148 \lambda^{1 / 4} n \stackrel{65}{>} 0 .
\end{aligned}
$$

We can now show that

$$
c\left(\left\{u_{2}, v_{1}\right\}\right)=\alpha \text { for every } v_{1} \in N_{G}^{\text {spec }}\left(u_{2}, \hat{U}_{1}\right) .
$$

Indeed, by (126), there exists $v_{2} \in N_{G}^{\text {typ }}\left(v_{1}, A_{2}\right)$. Note that $v_{2} \in A_{2}$ implies that $\left\{u_{1}, v_{2}\right\}$ is a special edge with color $\alpha$. Furthemore, both $u_{2} \in \bar{B}_{2}$ and $v_{2} \in A_{2}$ are good vertices, both $\left\{u_{2}, u_{1}\right\}$ and $\left\{v_{2}, v_{1}\right\}$ are typical edges, and both $\left\{u_{2}, v_{1}\right\}$ and $\left\{v_{2}, u_{1}\right\}$ are special edges. Thus, the 4-cycle $\left(u_{1}, u_{2}, v_{1}, v_{2}\right)$ is 2-special (cf. Definition 8.4), so Observation 8.5 guarantees that $c\left(\left\{u_{2}, v_{1}\right\}\right)=c\left(\left\{v_{2}, u_{1}\right\}\right)=\alpha$.

To extract the desired subset $B_{2} \subseteq \bar{B}_{2}$ from (125), we double-count

$$
Z=\left\{\left\{u_{2}, v_{1}\right\} \in E: u_{2} \in \bar{B}_{2}, v_{1} \in N_{G}^{\text {spec }}\left(u_{2}, I_{1}^{\text {bad }}\right) \text {, and } c\left(\left\{v_{1}, u_{2}\right\}\right) \neq \alpha\right\} .
$$

For each $u_{2} \in \bar{B}_{2}$, Statement (2) of Corollary 8.1 ensures that $E_{G}\left(u_{2}, U_{1}\right)$ admits at least

$$
\operatorname{deg}_{G}^{c}\left(u_{2}\right)-1-\left|U_{0} \backslash I_{0}^{\mathrm{bad}}\right| \stackrel{115}{=} \operatorname{deg}_{G}^{c}\left(u_{2}\right)-1-\left|\hat{U}_{0}\right|
$$

many special colors for $u_{2}$. By (127), every special edge $\left\{u_{2}, v_{1}\right\} \in E_{G}\left(u_{2}, \hat{U}_{1}\right)$ is colored $c\left(\left\{u_{2}, v_{1}\right\}\right)=\alpha$, which is forbidden in $Z$. Thus,

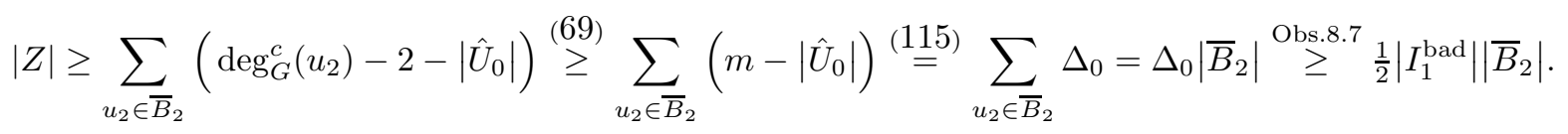

Averaging $|Z|$ over $I_{1}^{\text {bad }}$, we infer the existence of a vertex $v_{1} \in I_{1}^{\text {bad }}$ where

$$
B_{2}=B_{2}\left(v_{1}\right)=\left\{u_{2} \in N_{G}^{\text {spec }}\left(v_{1}, \bar{B}_{2}\right): c\left(\left\{u_{2}, v_{1}\right\}\right) \neq \alpha\right\}
$$

satisfies

$$
\left|B_{2}\right| \geq \frac{1}{2}\left|\bar{B}_{2}\right| \stackrel{125}{\geq} \frac{1}{2}\left(\frac{1}{6}-110 \lambda^{1 / 4}\right) n
$$

Consider the sets $A_{2}=A_{2}\left(u_{1}\right)$ and $B_{2}=B_{2}\left(v_{1}\right)$ from (124) and (128). Since $B_{2} \subseteq \bar{B}_{2}$ where $A_{2} \cap \bar{B}_{2}=\emptyset$ from (125), we infer that $A_{2} \cup B_{2}$ is a disjoint union of size

$\left|A_{2} \cup B_{2}\right|=\left|A_{2}\right|+\left|B_{2}\right| \stackrel{124}{\geq} \frac{n}{11}+\left|B_{2}\right| \stackrel{\frac{129}{\geq}}{\geq} \frac{n}{11}+\frac{1}{2}\left(\frac{1}{6}-110 \lambda^{1 / 4}\right) n=\left(\frac{23}{132}-55 \lambda^{1 / 4}\right) n \stackrel{\frac{651}{>}}{>}\left(\frac{1}{6}+186 \lambda^{1 / 4}\right) n$, which contradicts Corollary 8.6 , and concludes the proof of Fact 8.8

Fact 8.8 admits the following easy but useful corollary.

\footnotetext{
${ }^{3}$ Strictly speaking, the set $B_{2}$ we will define below will be a subset of that in the hypothesis of Corollary 8.6
} 
Corollary 8.9. Let $\ell \equiv 1(\bmod 3)$, and fix an amenable element $j \in \mathbb{Z}_{3}$. Fix an integer $\Delta \geq \max \left\{1, \Delta_{j}\right\}$, and fix $W_{j+1} \subseteq U_{j+1}$ of size $\left|W_{j+1}\right|<n /(100)$. Then

$$
\left|E_{G}^{\mathrm{spec}}\left(W_{j+1}, U_{j+2}^{\text {good }}\right)\right| \leq \frac{3}{10} \Delta n
$$

where $E_{G}^{\text {spec }}\left(W_{j+1}, U_{j+2}^{\text {good }}\right)$ includes all $\left\{w_{j+1}, u_{j+2}\right\} \in E$ with $w_{j+1} \in W_{j+1}$ and $u_{j+2} \in N_{G}^{\text {spec }}\left(w_{j+1}, U_{j+2}^{\text {good }}\right)$.

Proof of Corollary 8.9, Let $\ell \equiv 1(\bmod 3)$. Fix an amenable element $j \in \mathbb{Z}_{3}$, and w.l.o.g. let $j=0$. Fix an integer $\Delta \geq \max \left\{1, \Delta_{0}\right\}$, and fix $W_{1} \subseteq U_{1}$ of size $\left|W_{1}\right|<n /(100)$. Then

$$
\begin{aligned}
& \left|E_{G}^{\text {spec }}\left(W_{1}, U_{2}^{\text {good }}\right)\right|=\sum_{w_{1} \in W_{1}} \operatorname{deg}_{G}^{\text {spec }}\left(w_{1}, U_{2}^{\text {good }}\right)=\sum_{w_{1} \in W_{1} \cap \hat{U}_{1}} \operatorname{deg}_{G}^{\text {spec }}\left(w_{1}, U_{2}^{\text {good }}\right) \\
& +\sum_{w_{1} \in W_{1} \cap I_{1}^{\text {bad }}} \operatorname{deg}_{G}^{\text {spec }}\left(w_{1}, U_{2}^{\text {good }}\right) \stackrel{\text { Fct } 8.8}{\leq}\left|W_{1} \cap \hat{U}_{1}\right|\left(2 \Delta_{0}+5\right)+\left|W_{1} \cap I_{1}^{\text {bad }}\right| \frac{n}{10} \\
& \quad \leq\left|W_{1}\right|\left(2 \Delta_{0}+5\right)+\left|I_{1}^{\text {bad }}\right| \frac{n}{10} \stackrel{\text { Obs } 8.7}{\leq}\left|W_{1}\right|\left(2 \Delta_{0}+5\right)+\frac{n}{5} \Delta_{0} \leq \frac{n}{100}(2 \Delta+5)+\frac{n}{5} \Delta,
\end{aligned}
$$

and the quantity above is at most $27 \Delta n / 100$.

8.3. Proof of Lemma 2.9 in the case $\ell \equiv \mathbf{1}(\bmod 3)$. We now prove Lemma 2.9 in the case $\ell \equiv 1$ (mod 3). For this case, recall from (66) that the hypotheses of Lemma 2.9 assume

$$
\delta^{c}(G) \geq \begin{cases}(n+5) / 3 & \text { in Statement }(1) \text { with } \ell \equiv 1(\bmod 3) \\ (n+4) / 3 & \text { in Statement }(2) \text { with } \ell \equiv 1(\bmod 3) .\end{cases}
$$

We begin our work with Statement (2).

8.3.1. Statement (2) of Lemma 2.9. Statement (2) of Lemma 2.9 seeks to conclude that $(G, c)$ admits a properly colored $\ell$-cycle $C_{\ell}$. To prove this, we proceed by fixing an amenable element $j \in \mathbb{Z}_{3}$ from Observation 8.7. We first claim that

$$
E_{G}^{\text {spec }}\left(U_{j+1}^{\text {good }}, U_{j+2}^{\text {good }}\right) \neq \emptyset .
$$

To prove (131), we consider the identity

$$
\left|E_{G}^{\mathrm{spec}}\left(U_{j+1}, U_{j+2}^{\mathrm{good}}\right)\right|=\left|E_{G}^{\mathrm{spec}}\left(U_{j+1}^{\mathrm{bad}}, U_{j+2}^{\mathrm{good}}\right)\right|+\left|E_{G}^{\mathrm{spec}}\left(U_{j+1}^{\text {good }}, U_{j+2}^{\text {good }}\right)\right| .
$$

We will use Corollary 8.9 to bound $\left|E_{G}^{\mathrm{spec}}\left(U_{j+1}^{\mathrm{bad}}, U_{j+2}^{\text {good }}\right)\right|$, and we will use Corollary 8.1 to bound $\left|E_{G}^{\text {spec }}\left(U_{j+1}, U_{j+2}^{\text {good }}\right)\right|$. First, in the context of Corollary 8.9] we set $\Delta=1+\Delta_{j} \geq \max \left\{1, \Delta_{j}\right\}$, where we used $\Delta_{j} \geq 0$ from the amenability of $j \in \mathbb{Z}_{3}$. We also set $W_{j+1}=U_{j+1}^{\text {bad }}$, where

$$
\left|W_{j+1}\right|=\left|U_{j+1}^{\mathrm{bad}}\right| \stackrel{\frac{75}{\leq}}{\leq} 72 \lambda^{1 / 4} n \stackrel{\frac{65}{<}}{<} \frac{n}{100} .
$$

Consequently, Corollary 8.9 guarantees

$$
\left|E_{G}^{\mathrm{spec}}\left(U_{j+1}^{\mathrm{bad}}, U_{j+2}^{\text {good }}\right)\right| \leq \frac{3}{10} \Delta n .
$$

Second, Statement (2) of Corollary 8.1 guarantees that every $u_{j+2} \in U_{j+2}^{\text {good }}$ satisfies

$$
\begin{aligned}
\left|E_{G}^{\mathrm{spec}}\left(u_{j+2}, U_{j+1}\right)\right| \geq \operatorname{deg}_{G}^{c}\left(u_{j+2}\right)-1-\left|U_{j} \backslash I_{j}^{\mathrm{bad}}\right| \stackrel{115]}{=} \operatorname{deg}_{G}^{c}\left(u_{j+2}\right)-1-\left|\hat{U}_{j}\right| \\
\\
\quad \stackrel{69 \mid}{\geq} m+1-\left|\hat{U}_{j}\right| \stackrel{\sqrt{695}}{=} 1+\Delta_{j}=\Delta,
\end{aligned}
$$

and so

$$
\left|E_{G}^{\mathrm{spec}}\left(U_{j+1}, U_{j+2}^{\mathrm{good}}\right)\right|=\sum_{u_{j+2} \in U_{j+2}^{\text {good }}} \operatorname{deg}_{G}^{\mathrm{spec}}\left(u_{j+2}, U_{j+1}\right) \geq \Delta\left|U_{j+2}^{\mathrm{good}}\right| .
$$


Applying (133) and (135) to (132) yields

$$
\Delta\left|U_{j+2}^{\text {good }}\right| \leq\left|E_{G}^{\text {spec }}\left(U_{j+1}, U_{j+2}^{\text {good }}\right)\right| \leq \frac{3}{10} \Delta n+\left|E_{G}^{\text {spec }}\left(U_{j+1}^{\text {good }}, U_{j+2}^{\text {good }}\right)\right|,
$$

and so

$$
\left|E_{G}^{\text {spec }}\left(U_{j+1}^{\text {good }}, U_{j+2}^{\text {good }}\right)\right| \geq \Delta\left(\left|U_{j+2}^{\text {good }}\right|-\frac{3}{10} n\right) \stackrel{\frac{75}{\geq}}{\geq} \Delta n\left(\frac{1}{3}-75 \lambda^{1 / 4}-\frac{3}{10}\right)>\Delta n\left(\frac{3}{100}-75 \lambda^{1 / 4}\right) \stackrel{655}{>} 0,
$$

where we used $\Delta=1+\Delta_{j} \geq 1$ from the amenability of $j \in \mathbb{Z}_{3}$. This proves (131).

To prove Statement (2) of Lemma 2.9, fix an edge $\left\{u_{j+1}, u_{j+2}\right\} \in E_{G}^{\text {spec }}\left(U_{j+1}^{\text {good }}, U_{j+2}^{\text {good }}\right)$ from (131), where $u_{j+1} \in U_{j+1}^{\text {good }}$ and $u_{j+2} \in U_{j+2}^{\text {good }}$. We claim that

$$
E_{G}^{\text {spec }}\left(N_{G}^{\text {typ }}\left(u_{j+2}, U_{j+1}\right), N_{G}^{\text {typ }}\left(u_{j+1}, U_{j+2}^{\text {good }}\right)\right) \neq \emptyset .
$$

If (136) holds, then it concludes our proof, as follows. Fix $\left\{v_{j+1}, v_{j+2}\right\} \in E$ of (136), where $v_{j+1} \in$ $N_{G}^{\text {typ }}\left(u_{j+2}, U_{j+1}\right)$ and $v_{j+2} \in N_{G}^{\text {typ }}\left(u_{j+1}, U_{j+2}^{\text {good }}\right)$. We first observe that $\left(u_{j+2}, v_{j+1}, v_{j+2}, u_{j+1}\right)$ is a $(j+2)$-special 4-cycle (cf. Definition 8.4). Indeed, $u_{j+2} \in U_{j+2}^{\text {good }}$ is good from (131) and $v_{j+2} \in U_{j+2}^{\text {good }}$ is good from (136). The edge $\left\{u_{j+2}, v_{j+1}\right\} \in E$ is typical because $v_{j+1} \in N_{G}^{\text {typ }}\left(u_{j+2}, U_{j+1}\right)$ from (136), and the edge $\left\{v_{j+2}, u_{j+1}\right\} \in E$ is typical because $v_{j+2} \in N_{G}^{\text {typ }}\left(u_{j+1}, U_{j+2}^{\text {good }}\right)$ from (136). The edge $\left\{u_{j+2}, u_{j+1}\right\} \in E$ is special from from (131), and the edge $\left\{v_{j+2}, v_{j+1}\right\} \in E$ is special from (136). Since the 4-cycle $\left(u_{j+2}, v_{j+1}, v_{j+2}, u_{j+1}\right)$ is $(j+2)$-special, Observation 8.5 guarantees that its edges receive precisely 3 -colors, where the special edges $\left(\left\{u_{j+2}, u_{j+1}\right\}\right)$ and $\left\{v_{j+2}, v_{j+1}\right\}$ match in color and the typical edges $\left\{u_{j+2}, v_{j+1}\right\}$ and $\left\{v_{j+2}, u_{j+1}\right\}$ do not. Thus, $\left(u_{j+2}, v_{j+1}, v_{j+2}, u_{j+1}\right)$ is a properly colored 4-cycle. Equivalently, $\left(u_{j+2}, u_{j+1}, v_{j+2}, v_{j+1}\right)$ is a strong properly colored 4-cycle which Proposition 7.1 extends to a strong properly colored $\ell$-cycle $C_{\ell}$, as promised by Statement (2) of Lemma 2.9 ,

To prove (136), we proceed similarly to (131), and begin by considering the identity

$$
\begin{aligned}
\left|E_{G}^{\text {spec }}\left(U_{j+1}, N_{G}^{\text {typ }}\left(u_{j+1}, U_{j+2}^{\text {good }}\right)\right)\right|=\left|E_{G}^{\text {spec }}\left(U_{j+1} \backslash N_{G}^{\text {typ }}\left(u_{j+2}, U_{j+1}\right), N_{G}^{\text {typ }}\left(u_{j+1}, U_{j+2}^{\text {good }}\right)\right)\right| \\
+\left|E_{G}^{\text {spec }}\left(N_{G}^{\text {typ }}\left(u_{j+2}, U_{j+1}\right), N_{G}^{\text {typ }}\left(u_{j+1}, U_{j+2}^{\text {good }}\right)\right)\right| .
\end{aligned}
$$

As before, Corollary 8.9 will bound the first summand of (137), and Corollary 8.1 will bound the left hand side of (137). First, we again set $\Delta=1+\Delta_{j} \geq 0$, but we now set $W_{j+1}=U_{j+1} \backslash N_{G}^{\text {typ }}\left(u_{j+2}, U_{j+1}\right)$. Since $u_{j+2} \in U_{j+2}^{\text {good }}$ is a good vertex, Proposition 6.2 guarantees

$$
\left|W_{j+1}\right|=\left|U_{j+1} \backslash N_{G}^{\mathrm{typ}}\left(u_{j+2}, U_{j+1}\right)\right| \leq 233 \lambda^{1 / 4} n \stackrel{65}{<} \frac{n}{100} .
$$

Consequently, Corollary 8.9 guarantees

$$
\begin{aligned}
\left|E_{G}^{\mathrm{spec}}\left(U_{j+1} \backslash N_{G}^{\mathrm{typ}}\left(u_{j+2}, U_{j+1}\right), N_{G}^{\mathrm{typ}}\left(u_{j+1}, U_{j+2}^{\text {good }}\right)\right)\right| \\
\left.\quad \leq \mid E_{G}^{\text {spec }}\left(U_{j+1} \backslash N_{G}^{\mathrm{typ}}\left(u_{j+2}, U_{j+1}\right), U_{j+2}^{\text {good }}\right)\right) \mid \leq \frac{3}{10} \Delta n .
\end{aligned}
$$

Second, and identically to (134) and (135),

$$
\begin{aligned}
& \left|E_{G}^{\mathrm{spec}}\left(U_{j+1}, N_{G}^{\mathrm{typ}}\left(u_{j+1}, U_{j+2}^{\text {good }}\right)\right)\right| \\
& =\sum_{v_{j+2} \in N_{G}^{\mathrm{typ}}\left(u_{j+1}, U_{j+2}^{\text {good }}\right)} \operatorname{deg}_{G}^{\mathrm{spec}}\left(v_{j+2}, U_{j+1}\right) \geq \Delta\left|N_{G}^{\mathrm{typ}}\left(u_{j+1}, U_{j+2}^{\text {good }}\right)\right| .
\end{aligned}
$$

Applying (138) and (139) to (137) yields

$$
\begin{array}{r}
\left|E_{G}^{\text {spec }}\left(N_{G}^{\text {typ }}\left(u_{j+2}, U_{j+1}\right), N_{G}^{\text {typ }}\left(u_{j+1}, U_{j+2}^{\text {good }}\right)\right)\right| \geq \Delta\left(\left|N_{G}^{\text {typ }}\left(u_{j+1}, U_{j+2}^{\text {good }}\right)\right|-\frac{3}{10} n\right) \\
\stackrel{\text { Cor 8.3 }}{\geq} \Delta\left(\operatorname{deg}_{G}^{c}\left(u_{j+1}, U_{j+2}^{\text {good }}\right)-3-\frac{3}{10} n\right) \stackrel{\text { 775 }}{\geq} \Delta\left(\left(\frac{1}{3}-76 \lambda^{1 / 4}\right) n-3-\frac{3}{10} n\right) \\
>\Delta n\left(\frac{3}{100}-76 \lambda^{1 / 4}\right) \stackrel{65}{>} 0,
\end{array}
$$


where we used that $\Delta=1+\Delta_{j} \geq 1$ and that $n$ is sufficiently large. This proves (136), and completes the proof of Statement (2) of Lemma 2.9.

8.3.2. Statement (1) of Lemma 2.9. Statement (1) of Lemma 2.9 assumes that $\delta^{c}(G) \geq(n+5) / 3$ and seeks to conclude that $(G, c)$ admit 4 a rainbow $\ell$-cycle $C_{\ell}$. The argument here is similar to that of the previous subsection, where in fact we build upon that same argument. For that, note that $\delta^{c}(G) \geq(n+5) / 3 \geq(n+4) / 3$ allows all conclusions of the previous subsection to hold for the amenable element $j \in \mathbb{Z}_{3}$. As before, let $\left\{u_{j+1}, u_{j+2}\right\} \in E_{G}^{\text {spec }}\left(U_{j+1}^{\text {good }}, U_{j+2}^{\text {good }}\right)$ be fixed. We first observe that

$$
\Delta_{j+2} \leq-1
$$

since

$$
\begin{aligned}
\left|\hat{U}_{j+2}\right| \geq \operatorname{deg}_{G}^{c}\left(u_{j+1}, \hat{U}_{j+2}\right)=\operatorname{deg}_{G}^{c}\left(u_{j+1}, U_{j+2} \backslash I_{j+2}^{\mathrm{bad}}\right) \stackrel{\operatorname{Cor}[8.1}{\geq} \operatorname{deg}_{G}^{c}\left(u_{j+1}\right)-1 \stackrel{\frac{69}{\geq}}{\geq} m+1 \\
\quad \Longrightarrow \quad-1 \geq m-\left|\hat{U}_{j+2}\right| \stackrel{115]}{=} \Delta_{j+2} .
\end{aligned}
$$

Second, we observe that

$$
n \equiv 2(\bmod 3) \quad \text { or } \quad \Delta_{j} \geq 1 .
$$

To argue (142),

$$
\text { we assume, on the contrary, that } n \not \equiv 2(\bmod 3) \text { and } \Delta_{j}=0 \text {. }
$$

From (143), we will conclude that $j+1 \in \mathbb{Z}_{3}$ is also amenable, whence (141) also holds for $j+1 \in \mathbb{Z}_{3}$, in which case $\Delta_{j+1+2}=\Delta_{j} \leq-1$ contradicts $\Delta_{j}=0$ of (143). To see that $j+1 \in \mathbb{Z}_{3}$ is amenable, we note from (115) that

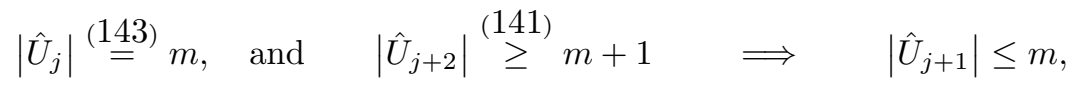

lest $3 m+2 \leq\left|\hat{U}_{0}\right|+\left|\hat{U}_{1}\right|+\left|\hat{U}_{2}\right| \leq n$ contradicts (143) (recall $m=\lfloor n / 3\rfloor$ from (69)). Thus,

$$
\Delta_{j+1} \stackrel{115}{=} m-\left|\hat{U}_{j+1}\right| \geq 0
$$

satisfies the first condition of amenability in Observation 8.7 Moreover,

$$
\begin{aligned}
\left|I_{j}^{\mathrm{bad}}\right|,\left|I_{j+2}^{\mathrm{bad}}\right| \leq \sum_{k \in \mathbb{Z}_{3}}\left|I_{k}^{\mathrm{bad}}\right| \stackrel{114,}{=} n-\sum_{k \in \mathbb{Z}_{3}}\left|\hat{U}_{k}\right| \stackrel{1153}{=} 1+\sum_{k \in \mathbb{Z}_{3}}\left(m-\left|\hat{U}_{k}\right|\right) \\
\stackrel{115]}{=} 1+\sum_{k \in \mathbb{Z}_{3}} \Delta_{k}=1+\Delta_{j}+\Delta_{j+1}+\Delta_{j+2} \stackrel{1411}{\leq} \Delta_{j}+\Delta_{j+1} \stackrel{143}{=} \Delta_{j+1} \stackrel{145}{\leq} 2 \Delta_{j+1},
\end{aligned}
$$

and so $\left|I_{j+2}^{\mathrm{bad}}\right| \leq 2 \Delta_{j+1}$ satisfies the second condition of amenability in Observation 8.7 Finally,

$$
\left|U_{j+3}\right|=\left|U_{j}\right| \stackrel{1144, \sqrt[115]{=}}{=}\left|\hat{U}_{j}\right|+\left|I_{j}^{\mathrm{bad}}\right| \stackrel{143}{=} m+\left|I_{j}^{\mathrm{bad}}\right| \stackrel{146)}{\leq} m+2 \Delta_{j+1} \leq m+2 \Delta_{j+1}+2,
$$

and so $\left|U_{j+3}\right| \leq m+2 \Delta_{j+1}+2$ satisfies the third condition of amenability in Observation 8.7

The remainder of our proof for Statement (1) of Lemma 2.9 splits into the two cases of (142). For these, recall that $\left\{u_{j+1}, u_{j+2}\right\} \in E_{G}^{\text {spec }}\left(U_{j+1}^{\text {good }}, U_{j+2}^{\text {good }}\right)$ was fixed at the start of this proof, where we now set $c\left(\left\{u_{j+1}, u_{j+2}\right\}\right)=\alpha$ for $\alpha \neq c_{u_{j+2}}$ on account that $\left\{u_{j+1}, u_{j+2}\right\}$ is a special edge.

Case $1(n \equiv 2(\bmod 3))$. We revisit (136) by confirming that

$$
\begin{aligned}
\emptyset \neq E_{G, \neg \alpha}^{\mathrm{spec}} & \left(N_{G}^{\mathrm{typ}}\left(u_{j+2}, U_{j+1}\right), N_{G}^{\mathrm{typ}}\left(u_{j+1}, U_{j+2}^{\text {good }}\right)\right) \\
& =\left\{\left\{v_{j+1}, v_{j+2}\right\} \in E_{G}^{\mathrm{spec}}\left(N_{G}^{\mathrm{typ}}\left(u_{j+2}, U_{j+1}\right), N_{G}^{\mathrm{typ}}\left(u_{j+1}, U_{j+2}^{\text {good }}\right)\right): c\left(\left\{v_{j+1}, v_{j+2}\right\}\right) \neq \alpha\right\},
\end{aligned}
$$

\footnotetext{
${ }^{4}$ Throughout our proof, we have assumed in (67) that $(G, c)$ avoids rainbow $\ell$-cycles $C_{\ell}$. Finding one now shows that our assumption (67) is flawed.
} 
where the set above consists of those edges of (136) which are not colored $\alpha$. If true, then any $\left\{v_{j+1}, v_{j+2}\right\} \in E$ of (147) gives a strong rainbow 4-cycle $\left(u_{j+2}, u_{j+1}, v_{j+2}, v_{j+1}\right)$ (recall Observation 8.5) which Proposition 7.1 extends to a strong rainbow $\ell$-cycle $C_{\ell}$, as promised by Statement (1) of Lemma 2.9. To see (147), we replay the details of (137)-(140) with the added hypothesis $n=3 m+2$. We again have

$$
\begin{aligned}
\left|E_{G, \neg \alpha}^{\mathrm{spec}}\left(U_{j+1}, N_{G}^{\mathrm{typ}}\left(u_{j+1}, U_{j+2}^{\text {good }}\right)\right)\right|=\left|E_{G, \neg \alpha}^{\mathrm{spec}}\left(U_{j+1} \backslash N_{G}^{\mathrm{typ}}\left(u_{j+2}, U_{j+1}\right), N_{G}^{\mathrm{typ}}\left(u_{j+1}, U_{j+2}^{\text {good }}\right)\right)\right| & \\
& +\left|E_{G, \neg \alpha}^{\mathrm{spec}}\left(N_{G}^{\mathrm{typ}}\left(u_{j+2}, U_{j+1}\right), N_{G}^{\mathrm{typ}}\left(u_{j+1}, U_{j+2}^{\text {good }}\right)\right)\right|,
\end{aligned}
$$

where the left hand side and first summand of (148) are defined analogously to (147). Clearly,

$$
\begin{aligned}
& \left|E_{G, \neg \alpha}^{\mathrm{spec}}\left(U_{j+1} \backslash N_{G}^{\mathrm{typ}}\left(u_{j+2}, U_{j+1}\right), N_{G}^{\mathrm{typ}}\left(u_{j+1}, U_{j+2}^{\mathrm{good}}\right)\right)\right| \\
& \quad \leq\left|E_{G}^{\mathrm{spec}}\left(U_{j+1} \backslash N_{G}^{\mathrm{typ}}\left(u_{j+2}, U_{j+1}\right), N_{G}^{\mathrm{typ}}\left(u_{j+1}, U_{j+2}^{\text {good }}\right)\right)\right|^{\frac{138}{\leq}} \frac{3}{10} \Delta n .
\end{aligned}
$$

Moreover, $\delta^{c}(G) \geq(n+5) / 3$ for $n \equiv 2(\bmod 3)$ ensures $\delta^{c}(G) \geq m+3$, and so Statement $(2)$ of Corollary 8.1 guarantees that every $v_{j+2} \in U_{j+2}^{\text {good }}$ satisfies

$$
\begin{aligned}
\left|E_{G}^{\mathrm{spec}}\left(v_{j+2}, U_{j+1}\right)\right| \geq \operatorname{deg}_{G}^{c}\left(v_{j+2}\right)-1-\left|U_{j} \backslash I_{j}^{\mathrm{bad}}\right| \stackrel{115}{=} & \operatorname{deg}_{G}^{c}\left(v_{j+2}\right)-1-\left|\hat{U}_{j}\right| \\
& \geq m+2-\left|\hat{U}_{j}\right| \stackrel{115}{=} 2+\Delta_{j}=1+\Delta .
\end{aligned}
$$

Consequently, those edges $E_{G, \neg \alpha}^{\text {spec }}\left(v_{j+2}, U_{j+1}\right)$ above not colored $\alpha$ satisfy $\left|E_{G, \neg \alpha}^{\text {spec }}\left(v_{j+2}, U_{j+1}\right)\right| \geq \Delta$, and so similarly to (139), we have

$$
\left|E_{G, \neg \alpha}^{\text {spec }}\left(N_{G}^{\text {typ }}\left(u_{j+2}, U_{j+1}\right), N_{G}^{\text {typ }}\left(u_{j+1}, U_{j+2}^{\text {good }}\right)\right)\right| \geq \Delta\left|N_{G}^{\text {typ }}\left(u_{j+1}, U_{j+2}^{\text {good }}\right)\right| .
$$

Similarly to (140), we apply (149) and (151) to (148) to infer

$$
\left|E_{G, \neg \alpha}^{\text {spec }}\left(N_{G}^{\text {typ }}\left(u_{j+2}, U_{j+1}\right), N_{G}^{\text {typ }}\left(u_{j+1}, U_{j+2}^{\text {good }}\right)\right)\right| \geq \Delta\left(\left|N_{G}^{\text {typ }}\left(u_{j+1}, U_{j+2}^{\text {good }}\right)\right|-\frac{3}{10} n\right) \stackrel{140}{>} 0,
$$

which proves (147).

Case $2\left(\Delta_{j} \geq 1\right)$. We again confirm (147), in which case any $\left\{v_{j+1}, v_{j+2}\right\} \in E(G)$ of (147) gives a strong rainbow 4-cycle $\left(u_{j+2}, u_{j+1}, v_{j+2}, v_{j+1}\right)$ which Proposition 7.1 extends to a strong rainbow $\ell$-cycle $C_{\ell}$, as promised by Statement (1) of Lemma 2.9. We again replay the details of (148)-(152), only this time we set $\Delta=\Delta_{j} \geq 1$ (as opposed to before, when $\Delta=1+\Delta_{j}$ ). Then (149) is updated to say that

$$
\begin{aligned}
& \left|E_{G, \neg \alpha}^{\mathrm{spec}}\left(U_{j+1} \backslash N_{G}^{\mathrm{typ}}\left(u_{j+2}, U_{j+1}\right), N_{G}^{\mathrm{typ}}\left(u_{j+1}, U_{j+2}^{\text {good }}\right)\right)\right| \\
& \quad \leq\left|E_{G}^{\mathrm{spec}}\left(U_{j+1} \backslash N_{G}^{\mathrm{typ}}\left(u_{j+2}, U_{j+1}\right), N_{G}^{\mathrm{typ}}\left(u_{j+1}, U_{j+2}^{\text {good }}\right)\right)\right|^{\frac{(138)}{\leq}} \frac{3}{10} \Delta_{j} n,
\end{aligned}
$$

while (150) is updated to say that each $v_{j+2} \in U_{j+2}^{\text {good }}$ satisfies

$$
\left|E_{G}^{\text {spec }}\left(v_{j+2}, U_{j+1}\right)\right| \geq \operatorname{deg}_{G}^{c}\left(v_{j+2}\right)-1-\left|\hat{U}_{j}\right| \frac{69}{\geq} m+1-\left|\hat{U}_{j}\right|=1+\Delta_{j} .
$$

Consequently, (151) is updated to say

$$
\left|E_{G, \neg \alpha}^{\text {spec }}\left(N_{G}^{\mathrm{typ}}\left(u_{j+2}, U_{j+1}\right), N_{G}^{\mathrm{typ}}\left(u_{j+1}, U_{j+2}^{\text {good }}\right)\right)\right| \geq \Delta_{j}\left|N_{G}^{\mathrm{typ}}\left(u_{j+1}, U_{j+2}^{\text {good }}\right)\right| .
$$

We apply (153) and (154) to (148) to infer

$$
\left|E_{G, \neg \alpha}^{\mathrm{spec}}\left(N_{G}^{\mathrm{typ}}\left(u_{j+2}, U_{j+1}\right), N_{G}^{\mathrm{typ}}\left(u_{j+1}, U_{j+2}^{\mathrm{good}}\right)\right)\right| \geq \Delta_{j}\left(\left|N_{G}^{\mathrm{typ}}\left(u_{j+1}, U_{j+2}^{\mathrm{good}}\right)\right|-\frac{3}{10} n\right) \stackrel{140}{>} 0,
$$

where we used $\Delta_{j} \geq 1$ from Case 2. This confirms (147), and concludes our proof of Lemma 2.9. 


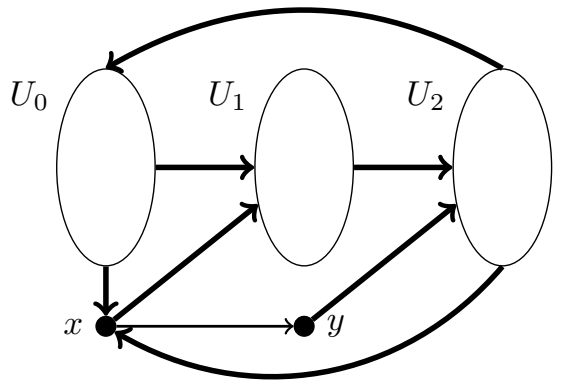

(A) The oriented graph $\vec{G}_{1}=\left(V, \vec{E}_{1}\right)$.

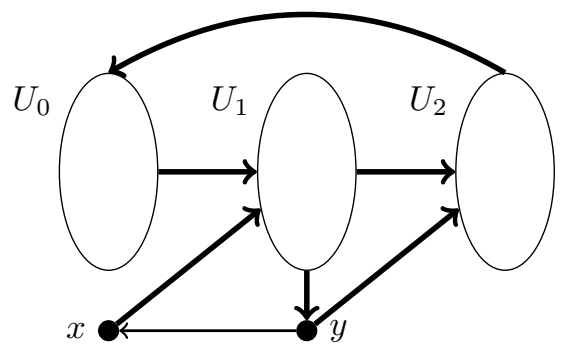

(B) The oriented graph $\vec{G}_{2}=\left(V, \vec{E}_{2}\right)$.

FiguRE 1. Neither $\vec{G}_{1}$ nor $\vec{G}_{2}$ admit directed $\ell$-cycles when $\ell \equiv 2(\bmod 3)$.

\section{Appendix: Proof-Sketch for Case 3 from the Introduction}

Recall the partition $V=U_{0} \cup U_{1} \cup U_{2} \cup\{x\} \cup\{y\}$ from Case 3 of Section 1.1. Let $\vec{G}_{1}=\left(V, \vec{E}_{1}\right)$ be the oriented graph whereby $(u, v) \in \vec{E}_{1}$ if, and only if, $(u, v)$ is an element of one of the following sets:

$$
U_{0} \times U_{1}, \quad U_{1} \times U_{2}, \quad U_{2} \times U_{0}, \quad\left(U_{0} \cup U_{2}\right) \times\{x\}, \quad\{x\} \times\left(\{y\} \cup U_{1}\right), \quad\{y\} \times U_{2} .
$$

Let $\vec{G}_{2}=\left(V, \vec{E}_{2}\right)$ satisfy $(u, v) \in \vec{E}_{2}$ if, and only if, $(u, v)$ is an element of one of the following sets:

$$
U_{0} \times U_{1}, \quad U_{1} \times U_{2}, \quad U_{2} \times U_{0}, \quad\{x\} \times U_{1}, \quad U_{1} \times\{y\}, \quad\{y\} \times\left(\{x\} \cup U_{2}\right) .
$$

One can see that neither $\vec{G}_{1}=\left(V, \vec{E}_{1}\right)$ nor $\vec{G}_{2}=\left(V, \vec{E}_{2}\right)$ admit directed $\ell$-cycles $\vec{C}_{\ell}$ when $\ell \equiv 2$ (mod 3). Recalling $(\hat{G}, \hat{c})$ from (5), construct $\hat{G}_{1} \subseteq \hat{G}$ by removing all edges between $U_{1}$ and $y$, and set $\hat{c}_{1}=\left.\hat{c}\right|_{E\left(\hat{G}_{1}\right)}$. Construct $\hat{G}_{2} \subseteq \hat{G}$ by removing all edges between $U_{0} \cup U_{2}$ and $x$, and set $\hat{c}_{2}=\left.\hat{c}\right|_{E\left(\hat{G}_{2}\right)}$. Then $E(\hat{G})=E\left(\hat{G}_{1}\right) \cup E\left(\hat{G}_{2}\right)$, and $\left(\hat{G}_{i}, \hat{c}_{i}\right), i=1,2$, is isomorphic to the edge-colored graph determined by $\vec{G}_{i}$. Therefore, a rainbow $\ell$-cycle of $(\hat{G}, \hat{c})$ can coincide entirely with neither $\hat{G}_{1}$ nor $\hat{G}_{2}$, and must admit an edge from $E\left(\hat{G}_{1}\right) \backslash E\left(\hat{G}_{2}\right)$ and an edge from $E\left(\hat{G}_{2}\right) \backslash E\left(\hat{G}_{1}\right)$. But this is impossible, because (5) ensures that every edge in the symmetric difference $E\left(\hat{G}_{1}\right) \triangle E\left(\hat{G}_{2}\right)$ is assigned the color $\star$.

\section{REFERENCES}

[1] Alon, N., Pokrovskiy, A., and Sudakov, B. (2017), "Random subgraphs of properly edge-coloured complete graphs and long rainbow cycles", Israel J. Math. 222, no. 1, 317-331.

[2] Alon, N., and Shapira, A. (2003), "Testing subgraphs in directed graphs", Proceedings of the thirty-fifth annual ACM symposium on Theory of Computing, ACM, 700-709.

[3] Caccetta, L., and Häggkvist, R. (1978), "On minimal digraphs with given girth", Congress. Numer. 21, $181-187$.

[4] Čada, R., Kaneko, A., Ryjáček, Z., and Yoshimoto, K. (2016), "Rainbow cycles in edge-colored graphs", Discrete Math. 339, no. 4, 1387-1392.

[5] Czygrinow, A., Molla, T., Nagle, B., Oursler, R., "On Odd Rainbow Cycles in Edge-Colored Graphs", submitted

[6] Erdős, P., Stone, A.H. (1946), "On the structure of linear graphs", Bulletin of the American Mathematical Society 52 (12), 1087-1091.

[7] Glock, S., and Joos, F. (2018), "A rainbow blow-up lemma", arXiv preprint arXiv:1802.07700

[8] Hladkỳ, J., Král', D., and Norin, S. (2017), "Counting flags in triangle-free digraphs", Combinatorica 37, no. 1, 49-76.

[9] Ji, Y., Wu., S., Song, H. (2018), "On short cycles in triangle-free oriented graphs", Czechoslovak Math. J. 68, no. 1, $67-75$.

[10] Keevash, P., Mubayi, D., Sudakov, B., and Verstraëte, J. (2007), "Rainbow Turán problems", Combin. Probab. Comput. 16, no. 1, 109-126.

[11] Kelly, L, Kühn, D., and Osthus, D. (2008), "A Dirac-type result on Hamilton cycles in oriented graphs", Combin. Probab. Comput. 17, no. 5, 689-709.

[12] Kelly, L., Kühn, D., and Osthus, D. (2010), "Cycles of given length in oriented graphs, J. Combin. Theory Ser. B 100, no. 3, 251-264. 
[13] Kim, J., Kühn, D., Kupavskii, A., and Osthus, D. (2018), "Rainbow structures in locally bounded colourings of graphs", arXiv preprint arXiv:1805.08424

[14] Kühn, D., Osthus, D., and Piguet, D. (2013), "Embedding cycles of given length in oriented graphs, European J. Combin. 34, no. 2, 495-501.

[15] Li, B., Ning, B., Xu, C., and Zhang, S. (2014), "Rainbow triangles in edge-colored graphs, European J. Combin. 36, 453-459.

[16] Li, H. (2013), "Rainbow $C_{3}$ 's and $C_{4}$ 's in edge-colored graphs", Discrete Math. 313, no. 19, $1893-1896$.

School of Mathematical and Statistical Sciences, Arizona State University, Tempe, AZ 85281, USA

E-mail address: aczygri@asu.edu

Department of Mathematics and Statistics, University of South Florida, Tampa, FL 33620, USA

E-mail address: molla@usf.edu

Department of Mathematics and Statistics, University of South Florida, Tampa, FL 33620, USA

E-mail address: bnagle@usf.edu

School of Mathematical and Statistical Sciences, Arizona State University, Tempe, AZ 85281, USA

E-mail address: Roy.Oursler@asu.edu 